\title{
BOIL, BOIL, TOIL AND TROUBLE: THE TROUBLE WITH BOIL WATER ADVISORIES IN BRITISH COLUMBIA
}

\author{
by \\ Renuka Grover
}

BSc., The University of British Columbia, 2004

A THESIS SUBMITTED IN PARTIAL FULFILMENT OF THE REQUIREMENTS FOR THE DEGREE OF

MASTER OF SCIENCE

in

THE FACULTY OF GRADUATE STUDIES

(Population and Public Health)

THE UNIVERSITY OF BRITISH COLUMBIA

(Vancouver)

April 2011

(C) Renuka Grover, 2011 


\section{Abstract}

Boil water advisories (BWAs) are public notifications of drinking water quality and are used as temporary, precautionary measures to protect the public from possible waterborne illnesses. British Columbia (BC) has among the highest numbers of BWAs and many have been in place for months to years, leading to the concern over their use as a 'band-aid' to water treatment and a substitute to action needed for its removal. With lengthier or on-again-off-again BWAs, there is concern that the public will become complacent and not comply with the BWA. Research on BWA is scarce and not much evidence is available to support practical decision making by the two groups of key players responsible for the management of BWAs - health authority officials and water suppliers.

Short-term and long-term BWAs from four of BC's five health authorities were randomly selected for investigation. Interviews of health authority officials and water suppliers were conducted to determine the decision-making process by which BWAs are issued; how BWAs are communicated to the public; and what consequential corrective action is taken after issuing, to progress towards the notification's removal. A total of 31 BWAs were investigated.

The decision-making processes varied considerably from case to case; different factors were considered depending on who was involved and the water system in question. The history of the water system, lack of water treatment, positive bacteriological water sample results and the type of water source were common criteria considered in the decision process. The majority of BWAs were communicated via personal interactions with the members of the public and public postings. Challenges with risk communication, message fatigue and public compliance were identified. Obstacles to the removal of longstanding BWAs included the lack of funding for infrastructure improvements or construction and technical challenges with the geographical remoteness of some small water systems.

Solutions that look to improving the overall management of small water systems in BC and that provide necessary guidance to decision-makers, before, during and after the issuing of BWAs, are needed for alleviating some of the challenges faced with BWAs. 


\section{Preface}

The study methodology, as described in the thesis manuscript, was reviewed and approved by the University of British Columbia's Behavioural Research Ethics Board (Certificate \#H08-02641).

The work presented in this thesis was conceived and conducted by the candidate, under the supervision, advice and guidance of the supervisory committee: Dr. Kay Teschke, Dr. Ray Copes and Dr. Donald Mavinic. The thesis manuscript was prepared by the candidate, with evaluation and feedback from the supervisory committee. 


\section{Table of Contents}

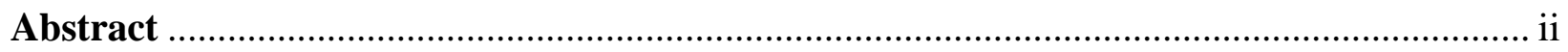

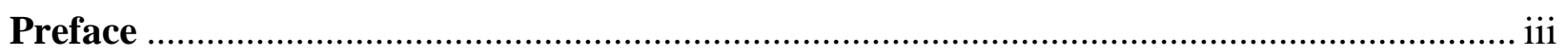

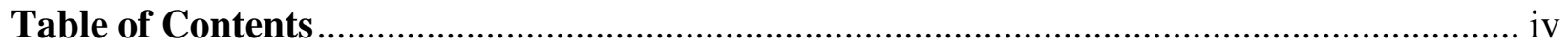

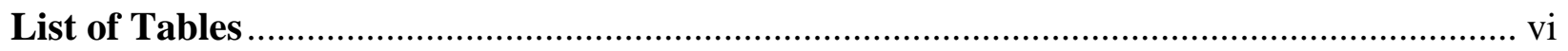

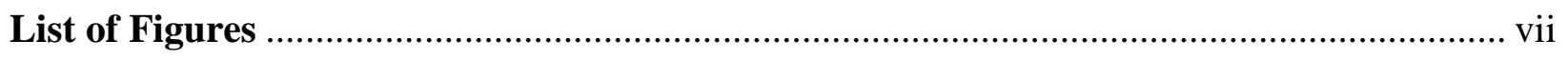

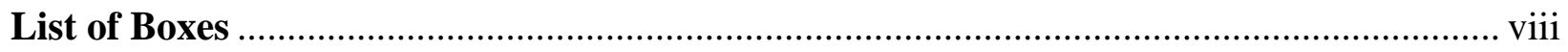

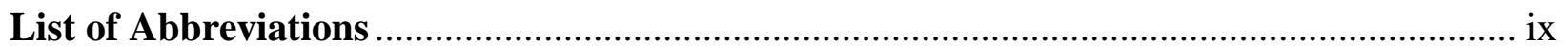

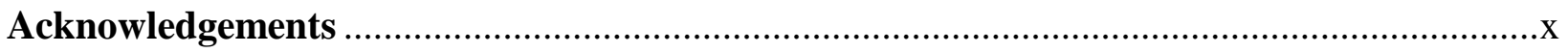

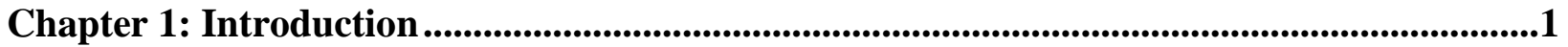

1.1 Overview of Rationale for the Thesis …..................................................................

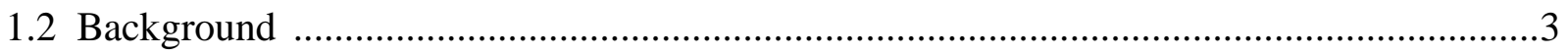

1.2.1 Drinking water \& public health .........................................................................

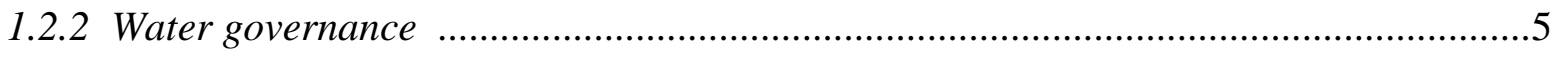

1.2.3 Public notifications of drinking water quality as a means to protect public health .....7

1.2.4 Boil water advisories .................................................................................................

1.2.5 Literature review \& knowledge gaps ................................................................. 8

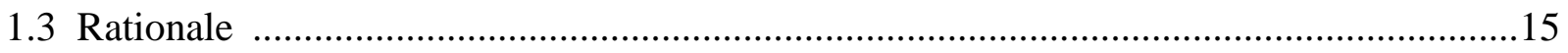

Chapter 2: The 'Trouble' with Boil Water Advisories in British Columbia ............................16

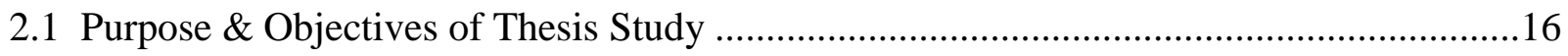

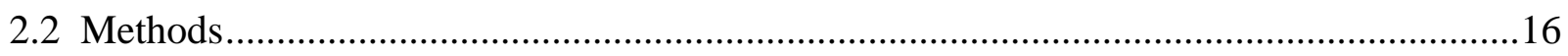

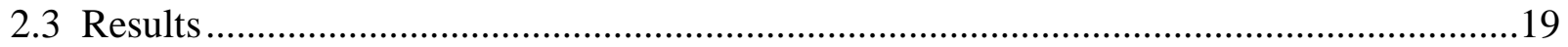

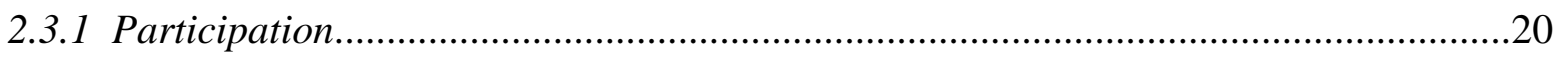

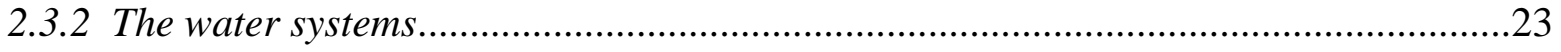

2.3.3 What's in a name? - A clarification of terminologies \& semantics............................33

2.3.4 To issue or not to issue, or not to issue? That is the question....................................36

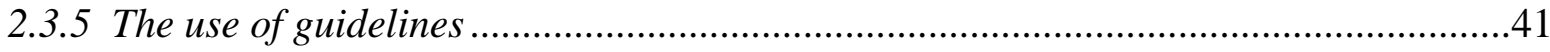

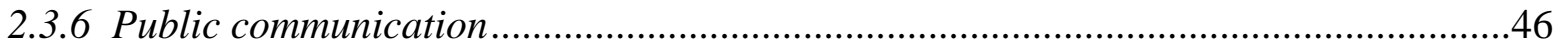

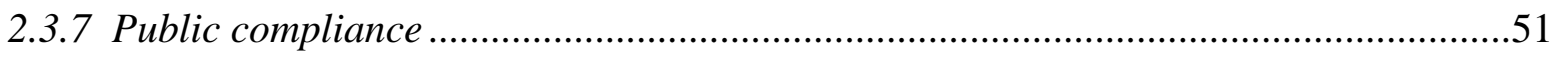

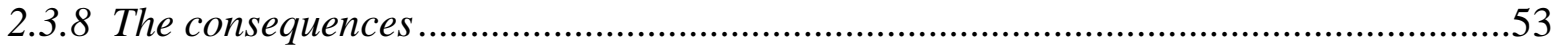

2.3.9 A snapshot of the current situation with boil water advisories in BC .......................54 
Chapter 3: Discussion of Results .56

3.1 Reasons to Issue Boil Water Advisories \& the Decision-Making Process ........................56

3.2 Public Communication of Boil Water Advisories \& Public Compliance..........................67

3.3 Actions in Effort to Rescind Boil Water Advisories.....................................................73

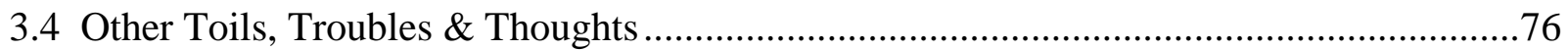

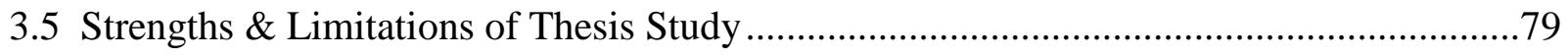

3.6 Significance of Thesis Research \& Concluding Remarks ............................................80

Chapter 4: Implications \& Ideas for Future Research ......................................................81

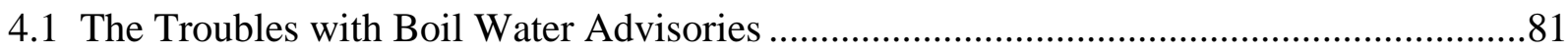

4.2 Potential Applications \& Knowledge Translation .....................................................85

4.2.1 UBC Program on Water Governance: Water security project toolkit........................88

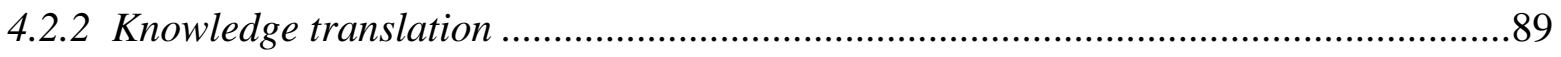

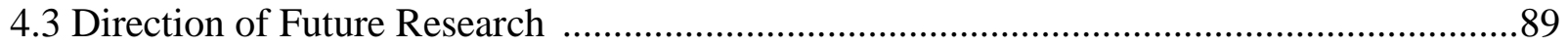

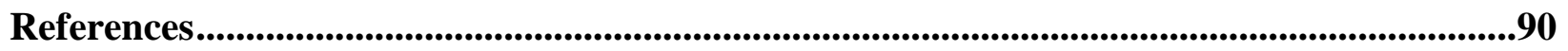

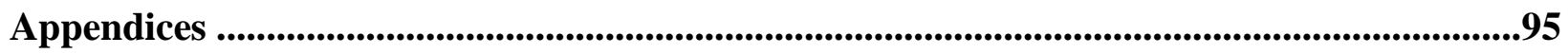

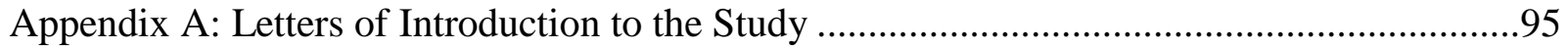

Appendix B: Interview Questionnaires with Quantitative Results ......................................99

Appendix C: Public/Water Consumer Interview Questionnaire with Quantitative Results ....119

Appendix D: Photograph from a Water System Visit......................................................129 


\section{List of Tables}

Table 1 Peer-reviewed articles, dissertations and grey literature on boil water advisories .........12

Table 2 The number of boil water advisories investigated in four health authorities in BC ......19

Table 3 Job titles of participants at the time of interview .....................................................22

Table 4 The story of each water system. Some main facts about the 31 water systems investigated in this study, by health authority

Table 5 Characteristics of all 31 randomly selected water systems under boil water advisory, and on the subset of 14 systems with reported information from both health authority officials and water suppliers. Percent agreement and kappas for measure of agreement beyond chance between health authority officials and water suppliers on information reported on the subset of 14 water systems

Table 6 The number of health authority officials and water suppliers who were at least partially correct in identifying and defining the three main types of public notifications, as per the Drinking Water Officer's Guide

Table 7 Reasons for issuing boil water advisories at all 31 water systems and at the 14 water systems with data from both health authority officials and water suppliers.....

Table 8 Reasons for issuing long-term versus short-term boil water advisories, as reported by 31 health authority officials ..... 38

Table 9 Individuals who were involved in the decision to issue boil water advisories

Table 10 A comparison of the content of regulations, policies and other supporting documents, as referenced by health authority officials, for use in guiding the management of public notifications, specifically the issuing and rescinding of BWAs.

Table 11 Information provided to public about the boil water advisory at a subset of water systems as reported by health authority officials and water suppliers water..... 


\section{List of Figures}

Figure 1 Number of boil water advisories in BC, by health authority, as of February 20, 2011 ..2

Figure 2 The trend in number of boil water advisories in BC over two decades ........................2

Figure 3 Number of participants (health authority officials and water suppliers) and gender

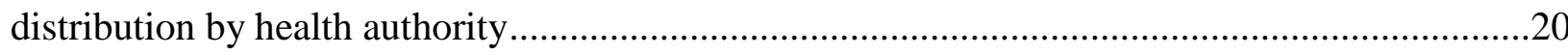

Figure 4 Why are boil water advisories primarily issued? A comparison of responses from

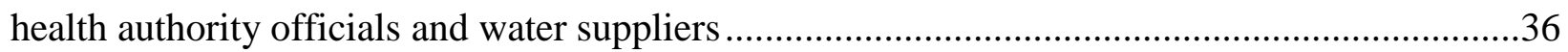

Figure 5 Decision-making criteria for issuing boil water advisories, as reported by health

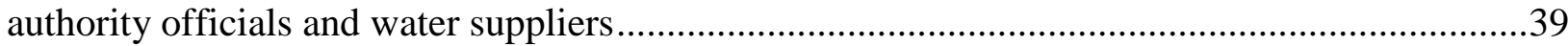

Figure 6a Do you follow written guidelines on when to issue boil water advisories? ...............42

Figure 6b Do you follow written guidelines on when to rescind boil water advisories? ...........42

Figure 7 Is there a template or checklist available that lists what information to include in a boil water advisory?

Figure 8 The means by which the boil water advisory was communicated to the public, as reported by health authority officials and water suppliers.....

Figure 9 Do you think the public complied with the boil water advisory? .............................51

Figure 10 Were any efforts taken to ensure public compliance?.

Figure 11 Common triggers leading to the issuing of boil water advisories, the specific actions required and the obstacles that hinder removal, as identified in this study. .55 


\section{List of Boxes}

Box 1 Terminology for public notifications of drinking water quality used by health authority officials .......

Box 2 The process for determining if a public notice is required and for rescinding a public

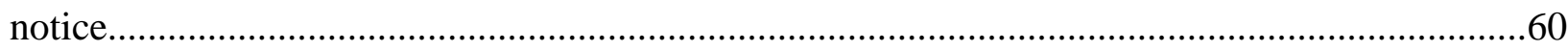




\section{List of Abbreviations}

$\begin{array}{ll}\text { BC } & \text { British Columbia } \\ \text { BWA } & \text { boil water advisory } \\ \text { DWO } & \text { Drinking Water Officer } \\ \text { DWPA } & \text { Drinking Water Protection Act } \\ \text { DWPR } & \text { Drinking Water Protection Regulation } \\ \text { E.coli } & \text { Escherichia coli } \\ \text { EHO } & \text { Environmental Health Officer } \\ \text { FHA } & \text { Fraser Health Authority } \\ \text { IHA } & \text { Interior Health Authority } \\ \text { MHO } & \text { Medical Health Officer } \\ \text { NHA } & \text { Northern Health Authority } \\ \text { UV } & \text { Ultra-violet } \\ \text { VCHA } & \text { Vancouver Coastal Health Authority } \\ \text { VIHA } & \text { Vancouver Island Health Authority } \\ \text { WHO } & \text { World Health Organisation }\end{array}$




\section{Acknowledgements}

Firstly, I would like to express my sincere gratitude to my supervisor, Dr. Kay Teschke, for her invaluable advice, guidance and patience over the years. I especially thank her for her support, encouragement and for making sure I stayed on track with my thesis work.

I would also like to thank Dr Ray Copes and Dr. Donald Mavinic for serving on my committee, sharing their unique perspectives and providing me with valuable feedback throughout the development of my thesis.

I am thankful to the Canadian Water Network for financial support and to the UBC Program on Water Governance research team for feedback and for providing me with the opportunity to translate my thesis findings into a toolkit for decision-makers.

I am grateful to the health authority officials and water suppliers who participated in this thesis research. Their time and insight was greatly appreciated. This study would not have been possible without them. I thank them for sharing their experiences and stories with me.

Last but not least, I would like to thank my family - my parents, my grandma, Raman and Sonika - for their unconditional support, encouragement and patience. I thank my parents for instilling a passion for higher education in me and always reminding me that I can accomplish anything I set my mind to. I also thank my brother, Raman, for setting the bar high, for inspiring and motivating me and for reminding me to relax! I feel truly blessed to have them all in my life. 


\section{Introduction}

\subsection{Overview of Rationale for the Thesis}

The provision of safe, clean drinking water is a fundamental component of public health. Boil water advisories (BWAs) and other forms of public notifications of drinking water quality are used as temporary, precautionary measures to protect the public from possible waterborne illnesses, primarily through recommending the boiling of tap water prior to use (1). In British Columbia (BC), BWAs are issued by water suppliers or health authority officials (such as Medical Health Officers or Drinking Water Officers) in situations ranging from higher than prescribed microbial counts in water samples, excessive turbidity to distribution system or treatment failures $(2,3)$.

There are more than 4500 public water systems in BC and of these, 515 are currently under a BWA ${ }^{1}(4,5)$. Thirteen BWAs were issued this year and the remaining 502 have been ongoing since before the start of the year (4). In fact, the longest running advisory was issued in 1988 on a water system in Prince Rupert; it is still ongoing - for over 8000 continuous days. Figure 1 shows the number of BWAs in BC by health authority and Figure 2 provides an historical account of the number of BWAs in BC since the mid 1980s. Over the years, the numbers of BWAs in BC have increased, largely due to better monitoring, increased reporting of systems with treatment failures or inadequate treatment, and identification of small water systems (systems serving up to 500 individuals) - which upon discovery are usually put on some form of public notification until evaluation can be completed $(3,6)$. The Interior Health Authority (IHA) has the highest number of BWAs, perhaps because it has a greater number of small water systems than other health authorities and a relatively large geographical area. The proportion of BC water systems under advisory does not reflect the proportion of the population affected, as a majority of BWAs are issued on small water systems which serve a relatively small fraction of the $\mathrm{BC}$ population (6). Approximately 3000 water systems in $\mathrm{BC}$ have fewer than 15 connections and $90 \%$ of BC's population receives water from 96 water systems serving large municipalities (5). Nevertheless, attention to the number and duration of BWAs in BC is warranted, especially since this province has among the highest numbers of advisories and gastrointestinal illness rates in all of Canada (1).

\footnotetext{
${ }^{1}$ As of February 20, 2011
} 


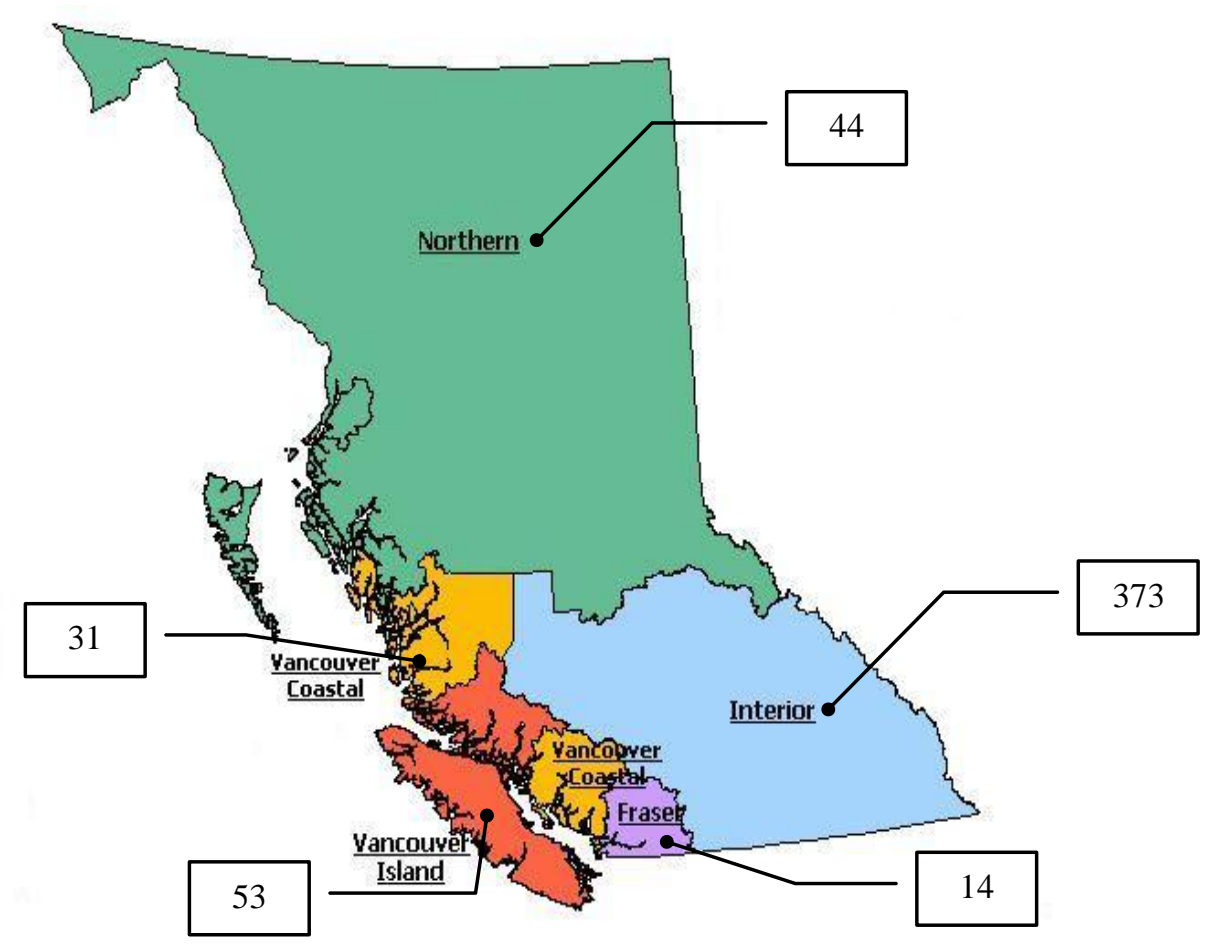

Figure 1: Number of boil water advisories in BC, by health authority, as of February 20, 2011

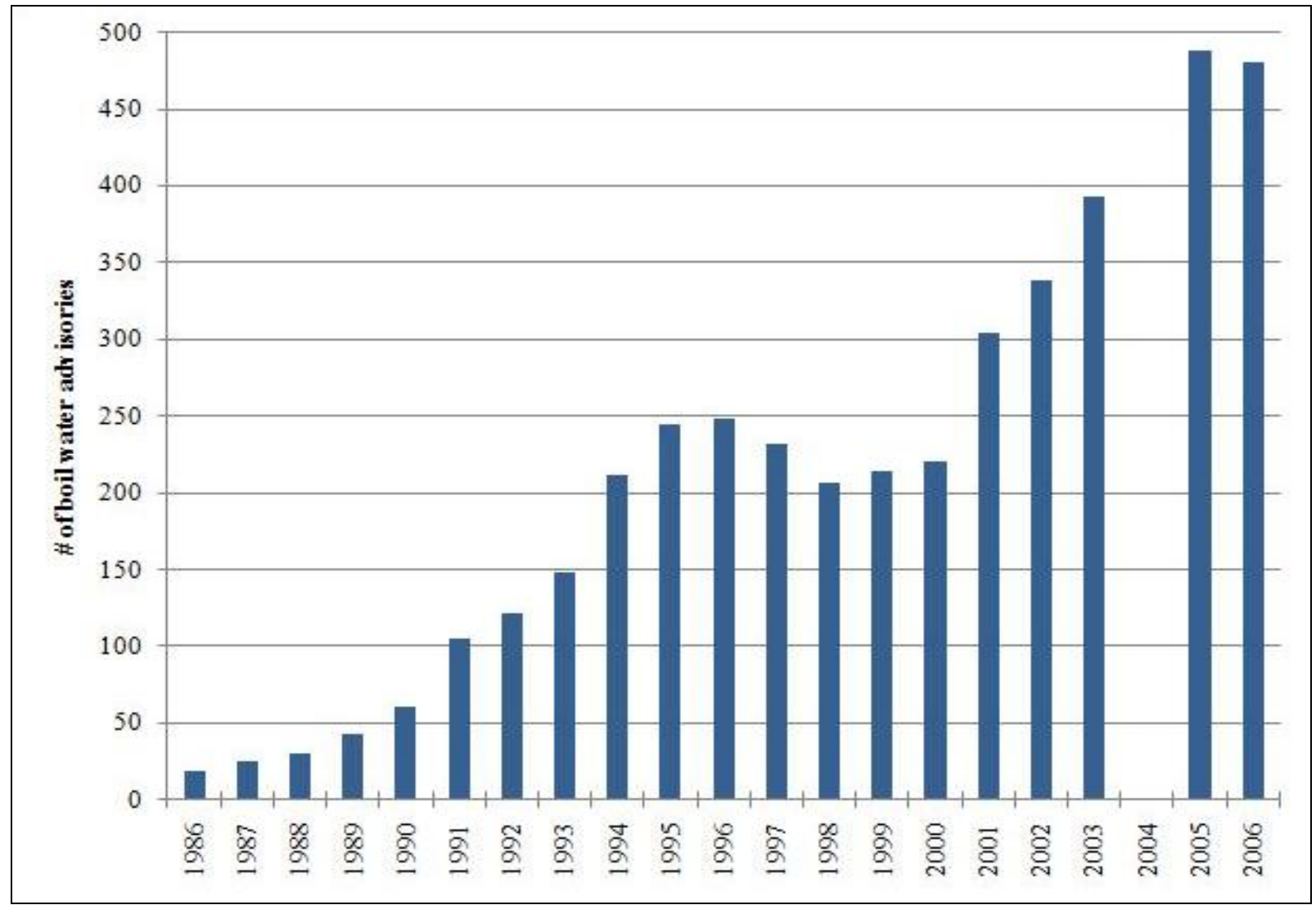

Figure 2: The trend in number of boil water advisories in $\mathrm{BC}$ over two decades *reproduced with permission from Barry Boettger (no data available for 2004) 
Although intended to be temporary, BWAs have persisted for weeks, months and years if what led to the decision to issue the public notification has not been addressed (2). The use of BWAs as a substitute for action needed to rescind the advisory is a matter of concern. With lengthier or 'on-again, off-again' BWAs, there is also concern that the public may become complacent and non-compliant with the notification's recommended risk reduction measures and may lose confidence in the system or the government (7).

The role of public notifications of drinking water quality and public health protection may be further hindered by confused terminology and inconsistent approaches across health authorities as well as by the paucity of research in this area. Of the various types of public notifications that exist, many have multiple terms that define the same form of notification $(8,9)$. The confusion with terminology is propagated with the use of imprecise wording when communicating public notices (10). With the scarcity of research on drinking water public notifications, not much scientific evidence is available to support practical decision-making processes and approaches used by health authority officials and water suppliers (10). As such, the ones who bear the responsibility for the systems are inevitably left to make risk management decisions at their own discretion, further challenged with having to act under uncertainty and under time sensitive conditions $(10,11)$. More research on public notifications of drinking water quality is therefore necessary. The thesis research discussed in this manuscript was developed with the hope that current problem areas, regarding public notifications of drinking water quality in BC, and specifically BWAs, would be elucidated and the results applied towards the development of practical solutions.

\subsection{Background}

\subsubsection{Drinking water \& public health}

Clean, safe and reliable drinking water is inarguably fundamental to public health, but in reality not all Canadians are continually supplied drinking water of such quality $(1,10,11)$. Canada has been home to some notorious waterborne events, most notably the highly publicised, Walkerton, Ontario Escherichia coli (E.coli) O157:H7 outbreak in May 2000, which resulted in more than 2300 cases of gastrointestinal illness and 7 deaths (12). Between 1974 and 2001, 288 waterborne outbreaks took place in Canada - many of these on semi-public water systems (privately-owned systems that serve the general public) (12). In British Columbia (BC), 29 
outbreaks took place between 1980 and 2004, in addition to tens of thousands of illnesses (12). Compared to the rest of Canada, $\mathrm{BC}$ has the highest rate of enteric disease, partly due to its greater number of unfiltered surface water sources and presence of waterborne parasites (13).

Waterborne illness - usually manifesting in the form of gastrointestinal illness - may result from various factors that cause water contamination $(14,15)$. Some main causes include, but are not limited to, natural hazards (such as heavy rainfall which can cause runoff and turbidity or naturally occurring arsenic in water supplies), system or distribution level failures (such as a broken chlorinator or deteriorating distribution pipes) or water operator errors or inadequacies (such as the lack of proper system maintenance) (15). Human action (example: industrialization) or the lack of action (example: poor water system management) may inadvertently produce risks to drinking water supplies $(15,16)$. All such factors become hazards as they directly or indirectly allow for the unwanted introduction of chemical or microbial contaminants into drinking water systems. Therefore the majority of efforts to protect public health from waterborne illnesses are based on measures to prevent such contamination from occurring and ensuring drinking water is safe $(13,17)$.

The importance of safe drinking water is discussed in countless places, but surprisingly, no Canadian legislation or regulation defines what is meant by 'safe' (10). The World Health Organization (WHO) defines 'safe drinking water' as water suitable for all domestic purposes and that does not present any significant health risk over a lifetime of consumption, including during periods of varying sensitivities (11). A more practical definition of quality drinking water (which presumably should be safe for use) is set out by Health Canada guidelines, recommending water to be free from any disease-causing organisms or harmful chemicals, radioactive compounds to be below defined thresholds, and physical parameters to be within acceptable ranges (13). It is important to recognize that safe drinking water is not water that is absolutely free of risk, as this next to impossible (10). Nevertheless, the aim should be to provide drinking water with a level of risk so negligible that an informed and reasonable individual perceives the water as safe $(10,15)$. 


\subsubsection{Water governance}

The World Health Organization (WHO) maintains that a safe, accessible and adequate supply of drinking water is a basic human right, essential to health and an important component of effective health protection policy (11). Further, the WHO, in the Guidelines for Drinking Water Safety, affirms that drinking water suppliers should be responsible for protecting consumers from possible water contamination events and for providing effective means to deal with them when they occur $(11,18)$.

In Canada, the responsibility for drinking water is spread across the federal, provincial/territorial and municipal governments (17). The federal government, in partnership with provinces and territories, developed the Guidelines for Canadian Drinking Water Quality as a means to provide benchmarks for safe drinking water (19). Since the Walkerton outbreak, public health officials in BC and throughout Canada have taken a more cautious approach in ensuring public health and drinking water quality $(1,6) \mathrm{BC}$ has adopted the nationally and internationally recommended multi-barrier approach to water safety $(6,17)$. This approach emphasizes management of drinking water supplies from 'source-to-tap' $(6,17)$. The implementation of multiple barriers or controls at various points in the system - at the water source, at the treatment plant, throughout the distribution system and at the consumer's tap - is the most effective means of preventing contamination (17). Also integral to BC's multi-barrier approach are regular water supply system assessments, system upgrading and maintenance, water quality monitoring and the training and certification of water suppliers (6).

In $\mathrm{BC}$, the authority for providing safe drinking water to the public lies with the Ministry of Health Services $(13,17)$. Prior to 1992, the Sanitary Regulation of the Health Act was in effect (13). In 1992 the Safe Drinking Water Regulation under the Health Act was introduced and required water suppliers, also known as water purveyors, to take responsibility for ensuring safe drinking water for their consumers $(3,13)$. In 2001, the Drinking Water Protection Act (DWPA) was enacted, but not put into force until May 2003 alongside the development of the Drinking Water Protection Regulation (DWPR) (3, 13). The DWPA/DWPR added emphasis on accountability and oversight and aimed for a more comprehensive and rigorous approach to ensure drinking water protection $(3,6)$. 
BC's five regional health authorities, via employed Drinking Water Officers (DWO), administer and enforce the DWPA, DWPR and Health Act and are responsible for the overall management, including surveillance and performance monitoring of BC's drinking water systems $(3,8)$. DWOs are Medical Health Officers (MHO) or those appointed by MHOs (3). The responsibilities of DWOs may be conducted by them, themselves, or in turn by those delegated in their place, such as Environmental Health Officers (EHO) - also sometimes referred to as Public Health Inspectors (8).

Public water systems which serve drinking water for domestic use to more than one single-dwelling family unit and have more than one physical connection ${ }^{2}$ to the water source fall under provincial-territorial jurisdiction $(20,21)$. This excludes water systems on national parks, First Nations reserves, Canadian Forces bases or other federal facilities, which are under federal jurisdiction (17). Also under provincial-territorial regulation are water systems that may have one physical connection but serve the general public, such as a water system solely connected to and serving a restaurant, a hotel or a daycare (20). Water suppliers are in charge of the ongoing operations and/or management of the drinking water system and may also be the owners of the system (8). In BC, water suppliers include local municipalities (as is the case for larger water systems, like the Greater Vancouver Water District), improvement districts (which commonly serve communities in rural areas), water user communities, restaurant, resort or campsite owners, and private individuals (which are more common to small water systems) (6). Water suppliers have the first level of responsibility to their consumers to notify them of any water quality or safety concerns and are legally required to do this, independently or under order from DWOs (3, $6)$.

The general public also bears some responsibilities with respect to their drinking water, such as conserving water, refraining from dumping hazardous substances down drains or ensuring potable water for themselves if they solely receive water from their own water source on private property (17) - but this topic and such private water systems fall outside the scope of the investigation described in this report.

\footnotetext{
2 "Connection" as per the Drinking Water Protection Regulation, means "the line from the water main to a dwelling, campsite or premises" (48)
} 


\subsubsection{Public notifications of drinking water quality as a means to protect public health}

Public notifications, in general, function to inform members of the public of an existing or impending health threat so that informed decisions on risk reduction behaviours can be made (22). Public notifications of drinking water quality, such as "boil water advisories" (BWA), "water quality advisories" and "do not use water advisories", are typically issued when a threat to water quality is, was or may be evident, and therefore act as preventative measures to protect public health from the possibility of contracting waterborne illnesses (9). A 'threat' to drinking water quality can be defined as any condition, circumstance or thing that results in the drinking water being supplied by a system as not potable (8). Potable water meeting standards as prescribed in the DWPR, is safe to drink and fit for domestic use by most consumers without need for additional treatment (8).

\subsubsection{Boil water advisories}

BWAs are issued in instances where the boiling of water, in accordance with specific requirements, renders the water safe for drinking (8). In addition to boiling water prior to use, BWAs may also warn the public to disinfect water, using chlorine bleach for instance, or to seek alternative sources of drinking water, such as bottled water (2). There is no defined situation in which a BWA should be issued nor any set criteria that determine whether a BWA should be issued or not $(8,10)$. In most cases, BWAs are issued when there is evidence of a potential threat to drinking water quality from poor source water quality, treatment or distribution equipment malfunction, inadequate disinfection or disinfection residuals, unacceptable microbiological quality of water, or excessively high turbidity (23). BWAs are also issued in situations where epidemiological evidence indicates water as the source of an outbreak (23). In general, BWAs that persist for longer periods of time are indicative of water systems that possess an insufficient infrastructure or that struggle with regulatory compliance (24). Shorter BWAs, on the other hand, tend to be issued under situations of uncertainty or during process control failures (24). There are also no criteria for when BWAs should be rescinded, but this is typically done when water quality has returned to acceptable levels or after operational malfunctions have been corrected and the distribution system flushed to displace residual contaminated water (23). 
BWAs can be issued by water suppliers and the regional health authorities (8). It is assumed that delegated statutory officials, such as DWOs, Public Health Engineers and EHOs/Public Health Inspectors, work in consultation with MHOs to ensure that appropriate public notifications are issued whenever necessary $(6,8)$. These health authority officials have the power to 'request' or 'order' water suppliers to give a public notice of water quality (8). An order is usually given when the water supplier does not agree with the issuing of a BWA or when the health authority official is uncertain whether the water supplier will comply with a request (8). The actual processes by which BWAs are issued and rescinded are not clear, but are known to vary from case to case (B. Boettger, Personal Communication, July 10, 2008). It is recommended that internal communication between involved parties be maintained throughout the process $(8,23)$. Some documentation and guidelines (at the federal, provincial-territorial level and local level) are available to support decision-making by health authorities, but their practical value and whether or not they are used consistently has not been previously examined.

BWAs are synonymous in function with "boil water alerts", "boil water notices" and "boil water orders" (3). There is much inconsistency and confusion with the terminology for describing the different forms for public notifications. For instance, within BC, three health authorities use the term BWA while the other two use the term "boil water notice" $(3,4)$. Clarity in definitions and terminology as well as consistency in appropriate use is therefore needed.

\subsubsection{Literature review \& knowledge gaps}

A literature review was done to determine the number and types of studies that have been conducted on public notifications of drinking water quality and specifically relating to BWAs. The health literature database, PubMed, and the environmental engineering database, Compendex - Engineering Village 2, were searched for peer-reviewed articles. Bibliographies of some discovered articles were also reviewed for related articles. Theses and dissertations were searched on ProQuest Dissertations and Theses and grey literature searched on the internet search engine, Google. Keywords included: boil water advisory, boil water notice, boil water order, and drinking water advisory (with wildcards to capture variants). In addition to the keywords, Google searches were attempted with Boolean search operators and Google search operators, such as filetype:pdf and site:gc.ca, to refine search results to a particular file type and URL, respectively. At most the first 10 pages of search results on Google were perused for grey literature. All English-language, relevant journal articles and theses or dissertations significantly 
relating to BWAs were included. Articles on waterborne outbreaks where a BWA was issued were excluded, unless there was substantial discussion about the BWA and not simply the mention that one was issued. Only English-language, Canadian grey literature was included. Table 1 shows the results of the literature search.

Scientific research on the public notification of drinking water quality is limited. There are a handful of articles and dissertations that focus on public compliance with public notifications of drinking water quality and some that describe specific case studies where BWAs were issued $(18,22,25-31)$. Of the studies that investigated compliance, most were conducted in waterborne outbreaks situations $(27,29,31)$. These studies have shown that noncompliance does occur, largely because the message is not received in time, is not properly understood or because of forgetting $(18,27,29,31)$. One interesting study on public compliance and awareness compared responses to BWAs between two towns in Oregon - one under a short-term advisory and one under a long-term advisory (22). This study showed that public compliance was greater in the town under a short-term advisory than the town under a long-term advisory (22). However, it is difficult to determine whether the observed difference in compliance is real, as the town under a long-term advisory was not specifically told to boil water, whereas the town under the short-term advisory was explicitly told what risk-reduction measures to take (22).

Two studies related to the decision to issue BWAs were identified. One investigated the value of monitoring data as an indicator for the need for a BWA and the other considered surveillance data $(32,33)$. The former study, which looked at Cryptosporidium monitoring data, proposed thresholds which may trigger the issuing of a BWA in outbreak situations, in conjunction with the presence of other risk factors, such as treatment equipment breakdown, high turbidity or illness in the community (33). The authors suggest that such monitoring data may also be utilised to assist in the decision to remove BWAs after the problems have been corrected (33). In the latter study, which investigated whether it would be beneficial to issue a BWA based on the spike in the sales on diarrheal remedies rather than wait for definitive water and clinical sample results, it was found that the best decision was to wait for definitive test results, unless the cost of issuing a BWA (assumed as the cost of bottled water consumed by the population affected at $\$ 1$ per day) is less than 13.92 cents per person per day (32). Such types of data are best regarded in conjunction with other risk factors, such as evidence of equipment breakdown or high turbidity (33). 
A 2008 report by the Technical Advisory Committee on Turbidity and Microbial Risk in Drinking Water, appointed by the Minister of Health, discussed the relationship between source water turbidity and risk for gastrointestinal illness and the value of using turbidity as a criterion for issuing BWAs (10). The Committee stressed the fact that no single reliable criterion exists that can determine whether a BWA should be issued or not; turbidity levels, therefore, cannot be consistently used for such purposes unless there is empirical evidence to support this for the specific water system in question (10). The Committee also commented on the need for a consistent, province-wide approach to issuing BWAs to help minimize the impression that each health authority is using different criteria and approaches (10). In addition, there is also a need for public notices to be communicated clearly, with precise language, as currently there are no standardized procedures on how BWAs should be best communicated to the public (10).

The grey literature identified in the search encompassed guidelines and reports, the latter of which emphasized the knowledge gaps that exist in this area $(6,10,20,34)$. Among the information gaps listed in the Provincial Health Officer's Annual Report in 2000, is the need for more information on the reasons behind issuing BWAs (20). This information gap was again mentioned in the Provincial Health Officer's 2007 report, The Progress on the Action Plan for Safe Drinking Water in BC (6). This report noted that the lack of a centralized and provincewide real-time tracking system of water quality data impedes $\mathrm{BC}$ from effective monitoring, research and improvements in water supplies (6). Other gaps identified include the lack of information on how many BWAs reflect temporary situations that can be quickly resolved and how many people are affected by BWAs (6).

Through personal communication with experts and public health professionals from the field, many other gaps were identified, some of which, if filled, would be of great practical value. The Provincial Drinking Water Officer expressed the need for a standardized decision framework, which considers numerous factors applicable to the water system under question, to assist in the decision-making process (B. Boettger, Personal Communication, July 10, 2008). There is also no information on public compliance towards and awareness of BWAs, particularly ones that are 'on-again, off-again' (B. Boettger, Personal Communication, July 10, 2008). Another health authority official was curious to know how the criteria and approaches used in deciding to issue BWAs compare between the five health authorities (E. Sigalet, Personal Communication, June 11, 2008). One other field expert expressed that any research that brings 
clarification to the present state of how BWAs are managed in BC would be of value, as right now there is much confusion and discrepancy (P. Bérubé, Personal Communication, June 3, 2008). 
Table 1: Peer-reviewed articles, dissertations and grey literature on boil water advisories

\begin{tabular}{|c|c|c|}
\hline Reference & Location & Topic \\
\hline \multicolumn{3}{|l|}{ Journal articles } \\
\hline $\begin{array}{l}\text { Anon. (1930) Canadian town responsible for deaths due to } \\
\text { polluted water (35) }\end{array}$ & $\begin{array}{l}\text { Kingsville, Ont., } \\
\text { Canada }\end{array}$ & $\begin{array}{l}\text { Discusses a typhoid epidemic where the town was held guilty of negligence } \\
\text { by court for inadequate risk communication of the BWA }\end{array}$ \\
\hline Mayon-White, et al. (1989) Boil the water (36) & $\begin{array}{l}\text { Oxford, } \\
\text { England, UK }\end{array}$ & $\begin{array}{l}\text { Commentary on lessons learned and recommendations pertaining to BWAs } \\
\text { after a cryptosporidiosis outbreak }\end{array}$ \\
\hline $\begin{array}{l}\text { Angulo, et al. (1997) A community waterborne outbreak of } \\
\text { salmonellosis and the effectiveness of a boil water order (29) }\end{array}$ & $\begin{array}{l}\text { Gideon, } \\
\text { Missouri, USA }\end{array}$ & $\begin{array}{l}\text { Survey of households on water consumption and compliance towards a } \\
\text { BWA during a salmonellosis outbreak }\end{array}$ \\
\hline $\begin{array}{l}\text { Harding, et al. (2000) Consumer response to public } \\
\text { notification }(22)\end{array}$ & Oregon, USA & $\begin{array}{l}\text { A comparison of water consumption and public compliance between two } \\
\text { communities on BWA - one under a short-term, emergency situation } \\
\text { (flooding) and one under a long-term, ongoing problem (filtration failure) - } \\
\text { and two control communities }\end{array}$ \\
\hline $\begin{array}{l}\text { O'Donnell, et al. (2000) Effect of a boil water notice on } \\
\text { behaviour in the management of a water contamination incident } \\
\text { (18) }\end{array}$ & $\begin{array}{l}\text { Great Britain, } \\
\text { UK }\end{array}$ & $\begin{array}{l}\text { Survey of households affected by sewage contamination to determine water } \\
\text { consumption and compliance towards BWA }\end{array}$ \\
\hline $\begin{array}{l}\text { Willocks, et al. (2000) Compliance with advice to boil drinking } \\
\text { water during an outbreak of cryptosporidiosis. Outbreak } \\
\text { Investigation Team (27) }\end{array}$ & $\begin{array}{l}\text { North Thames, } \\
\text { England, UK }\end{array}$ & $\begin{array}{l}\text { Survey of hospital employees in community under BWA on their } \\
\text { understanding of the BWA message and compliance during a } \\
\text { cryptosporidiosis outbreak }\end{array}$ \\
\hline $\begin{array}{l}\text { Wallis, et al. (2001) Application of monitoring data for } \\
\text { Giardia and Cryptosporidium to boil water advisories (33) }\end{array}$ & Ont., Canada & $\begin{array}{l}\text { Comparison of water parasite monitoring data from two municipalities } \\
\text { under BWA due to Giardia outbreak and assessment of whether such data } \\
\text { can be used to aid decisions on when to issue or rescind BWAs }\end{array}$ \\
\hline $\begin{array}{l}\text { Harrison, et al. (2002) Managing a large outbreak of } \\
\text { cryptosporidiosis: how to investigate and when to decide to lift } \\
\text { a 'boil water' notice ( } 37)\end{array}$ & $\begin{array}{l}\text { Devon, } \\
\text { England, UK }\end{array}$ & $\begin{array}{l}\text { A large cryptosporidiosis outbreak with a small discussion on the need for } \\
\text { criteria on rescinding BWA prior to its issuing, as a part of outbreak } \\
\text { management }\end{array}$ \\
\hline $\begin{array}{l}\text { Weber Jr., et al. (2003) Boil-water orders: Beneficial or } \\
\text { Hazardous? (38) }\end{array}$ & Michigan, USA & $\begin{array}{l}\text { Results of an experiment demonstrating that boiling water containing } \\
\text { thermally resistant bacterial spores may create an inhalation health hazard }\end{array}$ \\
\hline $\begin{array}{l}\text { Wagner, et al. (2005) Issue a boil-water advisory or wait for } \\
\text { definitive information? A decision analysis (32) }\end{array}$ & $\begin{array}{l}\text { Chicago, } \\
\text { Illinois, USA }\end{array}$ & $\begin{array}{l}\text { A decision-analysis based on whether to issue a BWA based on spiked sales } \\
\text { of diarrheal medicines or wait for water sample and human specimen } \\
\text { clinical results indicating cryptosporidiosis }\end{array}$ \\
\hline $\begin{array}{l}\text { Eggertson. (2008) Despite federal promises, First Nations' } \\
\text { water problems persist (39) }\end{array}$ & Canada & $\begin{array}{l}\text { Discusses the ongoing problem with drinking water in First Nations } \\
\text { communities, specifically the high number of persisting BWAs, and the lack } \\
\text { of action by federal government }\end{array}$ \\
\hline $\begin{array}{l}\text { Eggertson. (2008) Investigative report: } 1766 \text { boil-water } \\
\text { advisories now in place across Canada (1) }\end{array}$ & Canada & $\begin{array}{l}\text { A report highlighting and comparing the number of BWAs across Canada, } \\
\text { with emphasis on the need for government action. }\end{array}$ \\
\hline $\begin{array}{l}\text { Rundblad. (2008) The semantics and pragmatics of water } \\
\text { notices and the impact on public health }(40)\end{array}$ & $\begin{array}{l}\text { London, } \\
\text { England, UK }\end{array}$ & $\begin{array}{l}\text { A survey of undergraduate students' understanding of “do not drink } \\
\text { notices" and assessment of risk perception and likelihood of undertaking } \\
\text { specific risk reduction measures given a hypothetical scenario }\end{array}$ \\
\hline
\end{tabular}




\section{Reference}

Karagiannis, et al. (2009) Compliance with boil water advice following a water contamination incident in the Netherlands in 2007 (26)

Robertson, et al. (2009) A water contamination incident in Oslo, Norway during October 2007; a basis for discussion of boil-water notices and the potential for post-treatment contamination of drinking water supplies (41)

Rundblad, et al. (2010) Communication, perception and behaviour during a natural disaster involving a 'Do Not Drink' and a subsequent 'Boil Water' notice: a postal questionnaire study (25)

\section{Dissertation/Theses}

Anadu (1997) Factors affecting risk perception about drinking Oregon, USA

water and response to public notification (30)

Schell, et al. (1998) Separate worlds: One small town's compliance problems with water regulations (28)

Lacroix (2007) Impact of a boil water advisory on in-home water and food handling practices: A qualitative study (31)

Zumpano (2008) Small water systems in British Columbia: Possible obstacles and challenges in management (42)

\section{Canadian Grey Literature}

Province of BC (2001) A report on the health of British Columbians. Provincial Health Officer's annual report 2000. Drinking water quality in British Columbia: The public health perspective (20)

Losito. (2006) Report from the field: Vancouver's boil advisory In Disaster Management Canada (43)

Province of British Columbia (2007) Drinking Water Officer's Guide (8)

Province of British Columbia (2007) Progress on the Action Plan for Safe Drinking Water in British Columbia (6) Canada

\section{Location}

\section{Topic}

Holland,

Netherlands

Oslo, Norway

igation of water consumption habits and compliance among

households receiving drinking water from a system under BWA due to E.coli contamination

Description of a BWA event (from issue to removal) triggered by evidence of bacteria and parasites in water supplies and a discussion on the issuing of BWAs in response to the presence of Cryptosporidium and Giardia in water

Gloucestershire, A survey of households on a system under two consecutive water notices, UK due to flooding, to assess public compliance during both notification events

Assessment of risk perception of drinking water and determination of measures taken by public to protect health in response to a BWA in four communities

Colorado, USA Naturalistic study describing a town that failed to comply with federa drinking water standards, and the failure of townspeople to comply with a longstanding boil water order, due to personal, irrevocable beliefs on the purity of their drinking water and disagreement with health official's views Walkerton, ON, Case study on how some households reacted to the BWA, in terms of what behavioural changes they made (particularly in-home water and food handling), during the Walkerton, Ontario outbreak

BC, Canada A cross-case analysis of 6 small water systems in BC (half of which were on a permanent BWA) to obtain perspectives on governance structures and capacities to respond to risks to drinking water

\section{BC, Canada}

A review of current progress, information gaps and recommendations (some relating to BWAs)

Vancouver, BC, A personal account and field report, by the Regional Director of Health Canada Promotion, on the 2006 BWA in Vancouver, BC, including lessons learned

BC, Canada A document to provide policy and procedural guidelines to public health officials who must implement the DWPA/DWPR; includes information to support BWA decision making

A review of current progress, information gaps and recommendations (some relating to BWAs) 


\begin{tabular}{|c|c|c|}
\hline Reference & Location & Topic \\
\hline $\begin{array}{l}\text { Office of the Ombudsman. (2008) Fit to Drink: Challenges in } \\
\text { Providing Safe Drinking Water in British Columbia (3) }\end{array}$ & BC, Canada & $\begin{array}{l}\text { Results of an investigation into the public notification (BWAs) and } \\
\text { complaints process, with respect to drinking water, across BC. Concludes } \\
\text { with recommendations to health authorities to address identified problems }\end{array}$ \\
\hline $\begin{array}{l}\text { Province of British Columbia (2008) Progress on the Action } \\
\text { Plan for Safe Drinking Water in British Columbia (34) }\end{array}$ & BC, Canada & $\begin{array}{l}\text { A review of current progress, information gaps and recommendations (some } \\
\text { relating to BWAs); includes information about the } 2006 \text { BWA in Metro } \\
\text { Vancouver }\end{array}$ \\
\hline $\begin{array}{l}\text { Province of British Columbia. (2008) Turbidity and Microbial } \\
\text { Risk in Drinking Water (10) }\end{array}$ & BC, Canada & $\begin{array}{l}\text { A report reviewing risk communication and risk assessment issues having to } \\
\text { do with public notifications of drinking water quality (such as BWAs); } \\
\text { concludes with recommendations to the BC Minister of Health }\end{array}$ \\
\hline $\begin{array}{l}\text { Health Canada. (2009) Guidance for Issuing and Rescinding } \\
\text { Boil Water Advisories (23) }\end{array}$ & $\begin{array}{l}\text { Ottawa, ON, } \\
\text { Canada }\end{array}$ & $\begin{array}{l}\text { Documents factors that may be considered prior to issuing and rescinding } \\
\text { advisories and offers information for guidance during BWAs }\end{array}$ \\
\hline $\begin{array}{l}\text { Isfeld, H. (2009) Boil Water Advisory Mapping Project: An } \\
\text { exploration and review of available data (44) }\end{array}$ & $\begin{array}{l}\text { Winnipeg, MB, } \\
\text { Canada }\end{array}$ & $\begin{array}{l}\text { A report summarizing the availability of Canadian BWA data and its } \\
\text { limitations }\end{array}$ \\
\hline
\end{tabular}




\subsection{Rationale}

With the scarcity of research on drinking water public notifications, little scientific evidence is available to support practical decision-making processes and approaches used by health authority officials and water suppliers (10). Health authority officials and water suppliers are, therefore, left to make decisions at their own discretion $(10,11)$. While some policies and guidance materials have been developed, there is no information on whether they are understood and applied consistently by decision-makers.

Attention to BWAs in BC is warranted - particularly since the numbers are increasing, there is little information, clarity is needed, and since it appears, in some cases, that BWAs may be used in lieu of more permanent upgrades to water system infrastructure. This thesis aims to investigate the decision-making and processes before and after a BWA is issued. What criteria and decision-making processes are used by public health personnel and water suppliers in deciding to issue BWAs? Once issued, what is done to correct the problem and rescind the notification? How are BWAs communicated to the public? Why do certain BWAs persist for many years?

Short-term and long-term BWAs in four of BC's health authorities were investigated using structured interviews with closed and open-ended questions as the means to collect data. For each advisory, selected members of the two groups of key players involved in the management of BWAs - health authority officials and water suppliers - were interviewed. The methods of this investigation and its findings are reported in the following chapters of this manuscript. 


\section{The 'Trouble' with Boil Water Advisories in BC}

\subsection{Purpose \& Objectives of Thesis Study}

This thesis aimed to provide descriptive information on boil water advisories (BWA) in British Columbia (BC). Specifically, the objectives of this study were:

1) To examine the decision process by which health authority officials and water suppliers issue BWAs and the reasons for their issuance;

2) To determine by what methods BWAs are communicated to the public and the health authority's and water supplier's perceptions of public compliance; and

3) To determine what action is taken by health authority officials and water suppliers in an effort to rescind advisories.

\subsection{Methods}

Each of BC's five health authorities posts information on current BWAs on their websites (4). With the exception of the Interior Health Authority (IHA) which updates on a quarterly basis, information on BWAs are posted within hours of being issued (3). The amount of information publicly provided by each health authority varies, but all identify the name of the water system under advisory, the location (city) and the advisory start date.

Data collection was initiated by the random selection of short-term and long-term BWAs in the Fraser Health Authority (FHA), Vancouver Coastal Health Authority (VCHA), Interior Health Authority (IHA) and Northern Health Authority (NHA), using the most recently updated lists posted online. A short-term advisory was defined as a BWA that was ongoing at the time of selection and that was issued no more than 90 days from the date of project initiation. BWAs that were ongoing at that time and that were in place for longer than 6 months were considered long-term. The online lists for each health authority were copied onto Excel spreadsheets and randomly sorted. The top $10 \mathrm{BWAs}$ on the lists that qualified as either short-term or long-term were selected for inquiry. Although it was initially proposed to include all five of BC's health authorities in this study, Vancouver Island Health Authority (VIHA) was not included because of an extended approval process, and therefore no BWAs were selected for investigation from this health authority. 
Upon selection of the BWAs, personnel from the Drinking Water Program (45) for each $\mathrm{BC}$ health authority were contacted by telephone or email to identify the health authority official responsible for each of the selected BWAs. Full contact information of the health authority officials was acquired and the officials were subsequently contacted with an invitation to participate in the study (see Appendix A for a copy of the letter). Upon making contact with the health authority officials and after receiving their consent to participate, full contact information of the water suppliers was acquired. The water suppliers for the water systems under investigation were contacted and invited to participate. Initial contact was made through mail and/or email and follow-up contact was made via telephone. All participants were provided a copy of the interview questionnaire along with a letter describing the study and their rights as participants, and requesting participation. The selection of BWAs was initiated in August 2009 and data collection took place thereafter. Follow-up via telephone was initiated one week after primary contact and continued until contact was made or until July 15, 2010, the deadline established for data collection. If for any reason, further information on a randomly selected BWA was not obtainable or if the health authority official was not willing to participate, further investigation on that BWA was aborted, random selection was repeated and a replacement BWA was selected. The goal was to include at least 2 short-term and 2 long-term advisories from each health authority (a minimum of $16 \mathrm{BWAs}$ ), and to obtain interview data from both the health authority official and water supplier for each selected BWA. Therefore, the total number of BWAs selected for investigation in this thesis was increased in an attempt to meet this goal.

Health authority officials included in the study were required to be employees of the Province of British Columbia or their respective Health Authority and hold any of the following titles (or the equivalent): Medical Health Officer, Drinking Water Officer, Public Health Engineer, Public Health Inspector or Environmental Health Officer. Water suppliers were individuals deemed owners of the water system, the water system operator, those with whom health authority officials primarily kept contact with at the water system or those who the health authority official deemed most appropriate for participation in this study. Health authority officials or water suppliers who had retired or changed post as of January 1, 2009 were excluded from the study. 
In-person or telephone interviews were conducted using scripted, structured questionnaires that were very similar for the health authority and water supplier personnel (see Appendix B to view the questionnaires). Both health authority officials and water suppliers were asked about the process by which the decision to issue the BWA took place. Specifically, the criteria considered in deciding to issue as well as the rationale for issuing the BWA were investigated. Questions were asked to determine what actions had been taken since the issuing of the BWA to improve water quality and what obstacles, if any, were impeding the timely rescinding of the notification. The health authority officials and water suppliers were asked about the process by which the BWA was communicated to the public and their perception of public compliance towards the BWA. Also in the interview were questions on their personal opinions on water treatment and public notifications as well as some questions on the challenges faced during the public notification decision-making and communication process.

As part of the study, a randomly selected sample of individuals receiving water from the selected water systems under a BWA, were interviewed. The purpose was to obtain data on the effectiveness of public communication by the health authorities and/or the water suppliers and to assess public compliance towards the BWAs. The selected members of the public were asked questions to determine whether they were aware of the BWA and whether they understood the message. To assess compliance towards the BWA, participants were asked about any behavioural risk-reduction measures they had taken in response to the public notification. As the final sample size was very small, this investigation was excluded from the thesis, however, the interview questionnaire along with the tabulated results has been included in Appendix C.

Telephone interviews were audio-recorded using an electronic call recording software and device (Algo) and in-person interviews with a handheld tape recorder (Sony). The interview responses were transcribed into Microsoft Excel and Microsoft Word and coded for quantitative (and, where applicable, qualitative) analysis. Data analyses were done using S-PLUS (Version 8.0 for Windows, TIBCO Software Inc., Somerville, MA). 


\subsection{Results}

A total of 31 BWAs were investigated in this study; 18 of these were "long-term" and 13 "short-term". Table 2 summarizes the number of long-term and short-term BWAs selected by health authority. Also in this table are the range of durations of the BWAs and the range of start dates, to provide some insight into how long the advisories had been in place. The longest duration BWA investigated was issued on January 1, 1990; at the time of interview with the health authority official, it had been in place for more than 20 years.

Table 2: The number of boil water advisories investigated in four health authorities in BC

\begin{tabular}{|c|c|c|c|c|}
\hline Health Authority & Number of & $\overline{\mathrm{VAs}}$ & $\begin{array}{l}\text { Range in } \\
\text { duration" } \\
\text { (days) }\end{array}$ & $\begin{array}{c}\text { Range of BWA } \\
\text { start dates }\end{array}$ \\
\hline \multirow{3}{*}{$\begin{array}{c}\text { Fraser Health } \\
\text { Authority }\end{array}$} & Long-term: & 3 & $606-6420$ & $\begin{array}{c}\text { July } 1992- \\
\text { Auoust } 2008\end{array}$ \\
\hline & Short-term: & 5 & $43-59$ & $\begin{array}{l}\text { August } 2009- \\
\text { December } 2009\end{array}$ \\
\hline & Total: & 8 & & \\
\hline \multirow{3}{*}{$\begin{array}{c}\text { Interior Health } \\
\text { Authority }\end{array}$} & Long-term: & 3 & $2193-6594$ & $\begin{array}{l}\text { April } 1992- \\
\text { March } 2004\end{array}$ \\
\hline & Short-term: & 3 & $78-103$ & $\begin{array}{c}\text { December } 2009- \\
\text { February } 2010\end{array}$ \\
\hline & Total: & 6 & & \\
\hline \multirow{3}{*}{$\begin{array}{c}\text { Northern Health } \\
\text { Authority }\end{array}$} & Long-term: & 7 & $202-2557$ & $\begin{array}{c}\text { March } 2003- \\
\text { July } 2009\end{array}$ \\
\hline & Short-term: & 2 & $33-44$ & $\begin{array}{l}\text { February } 2010- \\
\quad \text { March } 2010\end{array}$ \\
\hline & Total: & 9 & & \\
\hline \multirow{3}{*}{$\begin{array}{c}\text { Vancouver } \\
\text { Coastal Health } \\
\text { Authority }\end{array}$} & Long-term: & 5 & $583-7423$ & $\begin{array}{c}\text { January } 1990- \\
\text { August } 2008\end{array}$ \\
\hline & Short-term: & 3 & $75-96$ & November 2009 \\
\hline & Total: & 8 & & \\
\hline \multicolumn{5}{|c|}{31 Boil Water Advisories } \\
\hline
\end{tabular}

* Duration at time of interview with health authority official. Some of the BWAs are still in place and ongoing today. 


\subsubsection{Participation}

In total, 38 individuals participated in the study; 24 were health authority officials ( 7 of whom were interviewed more than once because they were responsible for more than one randomly selected BWA) and 14 were water suppliers. Two health authority officials were interviewed in-person and the rest via telephone. 12 of the 14 water suppliers were interviewed via telephone; two water suppliers mailed or emailed their responses. Figure 3 illustrates the number of participants and their gender by health authority. $77 \%$ of the participating health authority officials and $64 \%$ of the water suppliers were male.

For each of the $31 \mathrm{BWAs}$, a health authority official was interviewed, but the water supplier was only interviewed for 14 of these systems. Therefore, there is a complete set of data for a subset of 14 water systems and BWAs, for which the health authority official and the water supplier were both interviewed. 17 water suppliers could not be interviewed: 13 refused to participate; and 4 could not be directly contacted or an interview time scheduled.

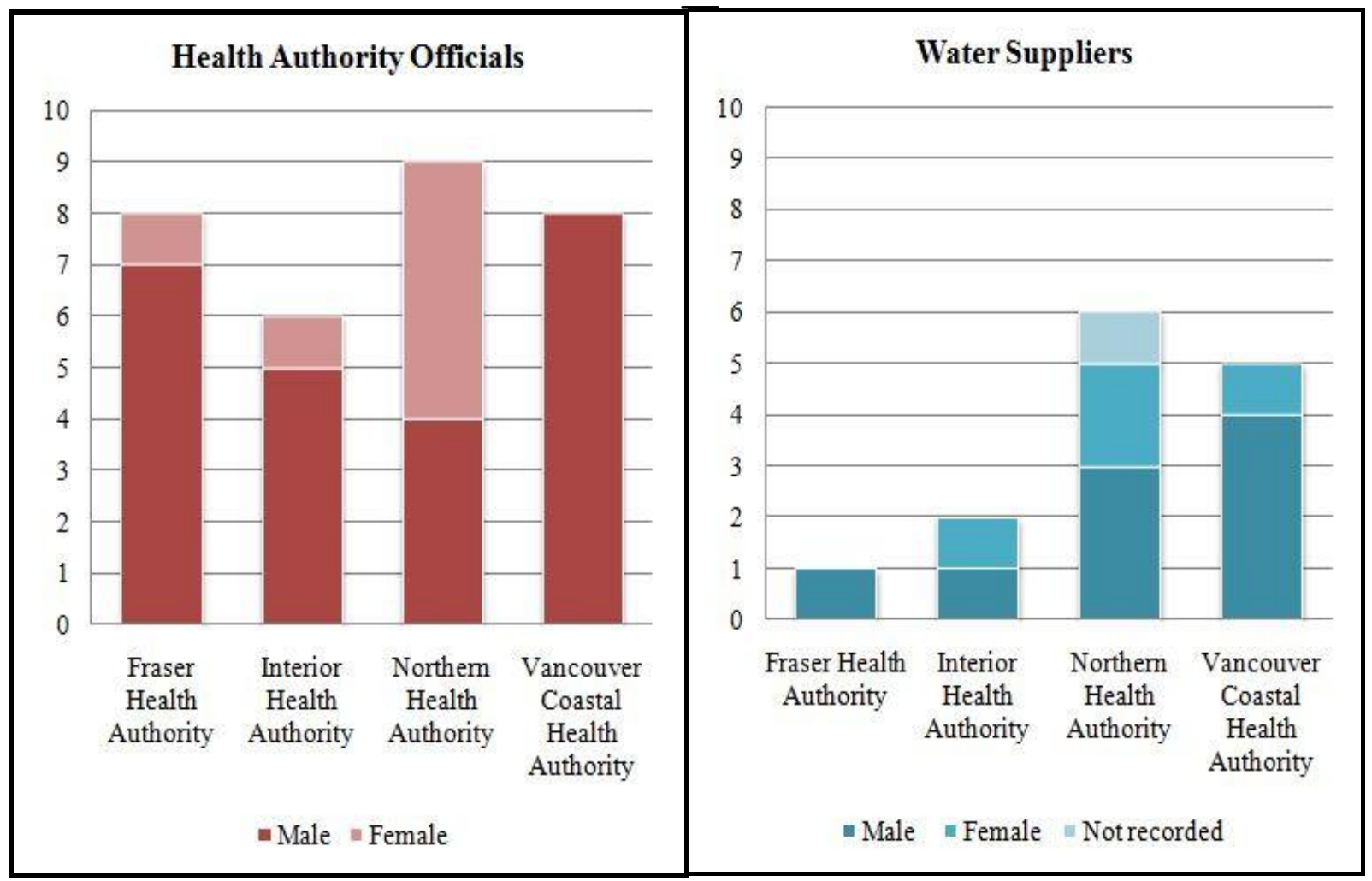

Figure 3: Number of participants (health authority officials and water suppliers) and gender distribution by health authority 
For three of the randomly selected BWAs, the health authority official in-charge could not participate (these BWAs were not investigated further and 3 other BWAs were re-selected for investigation in their place). Reasons for foregoing participation that were encountered included: discomfort with discussing the selected BWA and being unable to participate due to sensitive legal circumstances that involved the selected water system and the complexity of its ownership. One health authority official was away on extended medical leave and so another official temporarily overseeing his water systems was interviewed in his place. Other than that, all health authority officials interviewed were the ones designated and deemed responsible for the water system, at the time of the interview.

As few as 1 and as many as 21 phone calls were made in attempt to directly contact water suppliers for participation, post initial communication via mail or email. During the duration of the study, an interview could not be scheduled with four water suppliers. Two of these water suppliers were directly contacted but could not participate at the time of call and said to call back at another time; subsequent efforts to contact these water suppliers were unsuccessful and direct contact was unable to be made since. No contact at all was made with two water suppliers during the duration of the study; however, contact was made with individuals other than the water supplier, such as a spouse. One of these individuals stated that the water supplier was seriously ill and would likely not participate. Another individual stated that the water supplier discarded the study information and invitation and would not participate as he was leaving for vacation. Some attempts to directly speak with these water suppliers were still attempted, but to no avail.

Of the 13 water suppliers who refused to participate, 7 (54\%) stated they were not interested in participating. Thirty-eight percent (5/13) reported that they had no time or were busy and therefore did not want to participate. One of the $13(8 \%)$ could not participate due to serious illness and $2(15 \%)$ water suppliers were not able to participate as the water system was not under operation anymore and/or facing bankruptcy.

Table 3 lists the job titles of the health authority officials and water suppliers as selfreported by the participants. Thirteen of the thirty-one (42\%) health authority officials had a job title related to drinking water, such as Drinking Water Officer and nearly half of the water 
suppliers (43\%) reported a job title specific or linked to the water system. The majority of health authority officials were EHOs (84\%). The title of EHO was common to all health authorities; however, among participants, VCHA had a greater variety of job titles (such as Drinking Water Quality Specialist and Public Health Engineer) other than EHO. FHA was the only health authority with officials who were all EHOs. It is important to keep in mind that these observations were solely based on the sample of participants in this study and therefore may not be generalisable to all officials in the health authorities.

Table 3: Job titles of participants at the time of interview

\begin{tabular}{|l|l|}
\hline \multicolumn{1}{|c|}{ Health Authority Officials } & \multicolumn{1}{c|}{ Water Suppliers } \\
\hline Non-specific: & Non-specific: \\
- Environmental Health Officer & - Secretary of Strata Council \\
- Senior Environmental Health & - Process Management Consultant \\
Official & - Dock Manager \\
- Public Health Engineer & - Public Works Superintendant \\
& - Chair of Board \\
Water-specific: & - Facility Parks Operator \\
- Drinking Water Officer & - Manager of Utility Services \\
- Drinking Water Leader & - Informal/No formal title \\
- Drinking Water Quality Specialist & Water system-specific: \\
- Water Specialist & - Facility Manager \\
& - President of Water Users Community \\
& - Manager of Water Users Community \\
& - Co-owner of water system \\
& - Water Systems Operator \\
& - Owner of water system
\end{tabular}

The participating health authority officials had worked for an average of 9.6 years (minimum 0.17 years, maximum 22 years) in their current position at the time of the interview. The officials had at least 0.75 years and at most 36 years of experience with drinking water and drinking water programs, averaging 13.4 years. Of these years, health authority officials spent 0 - 9 years (average 1.7 years) doing nothing but drinking water, that is, focusing solely on drinking water related work during their career. 
Participating water suppliers reported working in their current position (or carrying out their current responsibility as 'water supplier') at the water system for $1-20$ years (average: 7.6 years). The water suppliers had an average of 7.0 years ( 0 - 25 years range) experience in the field of water management. Fifty percent of the water suppliers (7/14) self-reported some training or certification related to water system operations. At 10 of the 31 water systems, health authority officials reported that the water supplier had some training or certification.

\subsubsection{The water systems}

Thirty-one different water systems across the FHA, NHA, IHA and VCHA were investigated. Table 4 summarizes the details of each water system as reported in the health authority official and (where applicable) water supplier's interviews.

The water systems investigated consisted of those serving the public via private residences (examples: Water System-3 and Water-System-4) as well as those serving broader groups of the public via institutions or businesses (examples: Poultry Farm Water System-1 and Resort Water System-2). In some cases, the differing nature of service had determined the level of priority the system had over others. For example, the health authority official overseeing the BWA at Community Water System-23, required the system to immediately install a point-ofentry treatment device at the school post-issuance of the BWA, but was relatively lenient in requiring the same at the households connected to the system. Systems linked to institutions or businesses may have been subject to regulations other than those specific to drinking water (e.g., food handling regulations for restaurants). The additional regulations can provide an extra lever of control for the health authority.

Within the last 5 years, 8 of the 31 water systems had been under a BWA prior to the one being investigated in the study. Almost all of the water systems with this history of having been under a previous BWA were ones facing a short-term BWA at the time of interview. In 5 of these water systems (63\%), the BWA immediately prior to the one investigated was issued for the same reason as the current one. 
Table 4: The story of each water system. Some main facts about the 31 water systems investigated in this study by health authority

\begin{tabular}{|c|c|c|c|c|c|c|c|c|c|}
\hline & & $\begin{array}{l}\text { Water System } \\
\text { Name }\end{array}$ & Source $^{1}$ & $\begin{array}{c}\# \\
\text { Connections } \\
\end{array}$ & $\begin{array}{l}\text { \# Pop. } \\
\text { Served }\end{array}$ & $\begin{array}{c}\text { Central } \\
\text { Treatment }\end{array}$ & Reason for BWA & Actions & About the Water System \\
\hline & & $\begin{array}{l}\text { Poultry Farm Water } \\
\text { System - } 1\end{array}$ & $\begin{array}{l}\text { Deep } \\
\text { well }\end{array}$ & 4 & 6 & $\mathrm{UV}^{*}$ & $\begin{array}{l}\text { Repeated presence } \\
\text { of } E . \text { coli in water }\end{array}$ & $\begin{array}{l}\text { New UV treatment system } \\
\text { installed; BWA removal pending } \\
\text { negative water sample results }\end{array}$ & $\begin{array}{l}\text { Discovered in } 2006 \text { when owners applied for } \\
\text { food service permit. Family-run business; } \\
\text { water system serves the supplier's house and } \\
\text { farm. }\end{array}$ \\
\hline & ミ气 & $\begin{array}{l}\text { Resort Water } \\
\text { System }-2\end{array}$ & $\begin{array}{l}\text { Surface } \\
\text { (creek) }\end{array}$ & 10 & 50 & Chlorination & $\begin{array}{l}\text { High number of } \\
\text { total coliform } \\
\text { bacteria and E.coli- } \\
\text { positive water } \\
\text { samples }\end{array}$ & $\begin{array}{l}\text { Source inspection and close } \\
\text { monitoring of chlorine residuals. } \\
\text { Point-of-use filters installed at } \\
\text { cabin kitchen taps. BWA removal } \\
\text { pending negative water sample } \\
\text { results }\end{array}$ & $\begin{array}{l}\text { Discovered in 1994; Water system serves an } \\
\text { 8-cabin motel-type of resort. Main public } \\
\text { served is transient and the resort functions } \\
\text { seasonally. }\end{array}$ \\
\hline & $\frac{\pi}{2}$ & Water System - 3 & $\begin{array}{l}\text { Deep } \\
\text { well }\end{array}$ & 3 & 6 & None & $\begin{array}{l}\text { High repeated } \\
\text { presence of total } \\
\text { coliform in water }\end{array}$ & $\begin{array}{l}\text { No action since. Water supplier } \\
\text { required to install new water } \\
\text { treatment system. }\end{array}$ & $\begin{array}{l}\text { Developed in the } 1960 \text { s. Water system } \\
\text { serves water supplier's house and } 2 \text { rental } \\
\text { units. }\end{array}$ \\
\hline & & Water System - 4 & $\begin{array}{l}\text { Deep } \\
\text { well }\end{array}$ & 5 & 15 & $\begin{array}{l}\text { Filtration, } \\
\text { Chlorination }\end{array}$ & $\begin{array}{l}\text { High repeated } \\
\text { presence of total } \\
\text { coliform in water }\end{array}$ & $\begin{array}{l}\text { Repaired malfunctioning } \\
\text { chlorinator injection; BWA } \\
\text { removal pending negative water } \\
\text { sample results }\end{array}$ & $\begin{array}{l}\text { Approximately } 25 \text { year old system serving a } \\
\text { property of } 5 \text { homes. Maintained by a } \\
\text { management company and a contracted } \\
\text { water supplier. }\end{array}$ \\
\hline 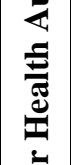 & & Water System - 5 & $\begin{array}{l}\text { Deep } \\
\text { well }\end{array}$ & 5 & 7 & None & $\begin{array}{l}\text { Presence of repeat } \\
\text { total coliform counts } \\
\text { in water samples }\end{array}$ & $\begin{array}{l}\text { No action since. Water supplier to } \\
\text { flush system, chlorinate reservoir } \\
\text { and submit more water samples. } \\
\text { Water supplier unwilling to } \\
\text { connect ozone treatment system. }\end{array}$ & $\begin{array}{l}\text { Water system built around an informal } \\
\text { society of homes. All homeowners } \\
\text { voluntarily share role of water supplier. } \\
\text { System has been put back on another BWA. }\end{array}$ \\
\hline 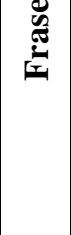 & & $\begin{array}{c}\text { Water Users' } \\
\text { Community Water } \\
\text { System - } 6\end{array}$ & $\begin{array}{l}\text { Surface } \\
\text { (lake) }\end{array}$ & 40 & 100 & None** & $\begin{array}{l}\text { Presence of E.coli in } \\
\text { water samples }\end{array}$ & $\begin{array}{l}\text { All homes have had a point-of- } \\
\text { use/point-of-entry treatment } \\
\text { device installed. BWA removal } \\
\text { pending negative water sample } \\
\text { results }\end{array}$ & $\begin{array}{l}\text { System discovered in } 1976.40 \text { vacation } \\
\text { homes forming a Water Users' Community } \\
\text { where each homeowner is part owner of } \\
\text { water system. Residents are onsite } \\
\text { seasonally. Volunteer water supplier takes } \\
\text { responsibilities of overall maintenance of } \\
\text { water system }\end{array}$ \\
\hline & 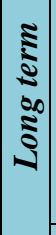 & $\begin{array}{l}\text { Water Users' } \\
\text { Community Water } \\
\text { System }-7\end{array}$ & $\begin{array}{l}\text { Surface } \\
\text { (spring) }\end{array}$ & 22 & 18 & None & $\begin{array}{l}\text { Presence of E.coli } \\
\text { in water samples }\end{array}$ & $\begin{array}{l}\text { No action taken or deemed } \\
\text { necessary as water system will } \\
\text { soon be integrated into a larger } \\
\text { one }\end{array}$ & $\begin{array}{l}\text { Water system developed in } 1948.22 \text { homes } \\
\text { forming a Water Users' Community. Chair } \\
\text { of community is water supplier. This water } \\
\text { system is moving towards integration with a } \\
\text { larger regional district's system; therefore, } \\
\text { the current BWA will be dissolved as will } \\
\text { this water system }\end{array}$ \\
\hline & & $\begin{array}{l}\text { Water Users' } \\
\text { Community Water } \\
\text { System }-8\end{array}$ & Mixed & 19 & 40 & None** & $\begin{array}{l}\text { Water system } \\
\text { without any water } \\
\text { treatment in place }\end{array}$ & $\begin{array}{l}18 / 19 \text { homes have a point-of- } \\
\text { use/point-of-entry device } \\
\text { installed; BWA removal pending } \\
\text { final home's installation with } \\
\text { device and proof of effectiveness } \\
\text { via negative water sample results }\end{array}$ & $\begin{array}{l}\text { Built in the } 1970 \text { 's and serves } 19 \text { homes. } \\
\text { Manager of Water Users' Community serves } \\
\text { as water supplier, although each household is } \\
\text { responsible for treatment. }\end{array}$ \\
\hline
\end{tabular}




\begin{tabular}{|c|c|c|c|c|c|c|c|c|c|}
\hline \multirow{7}{*}{ 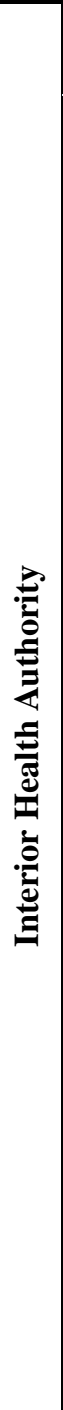 } & & $\begin{array}{c}\text { Water System } \\
\text { Name }\end{array}$ & Source $^{1}$ & $\begin{array}{c}\# \\
\text { Connections }\end{array}$ & $\begin{array}{l}\text { \# Pop. } \\
\text { Served }\end{array}$ & $\begin{array}{l}\text { Central } \\
\text { Treatment }\end{array}$ & Reason for BWA & Actions & About the Water System \\
\hline & \multirow{3}{*}{$\frac{\pi^{2}}{5}$} & $\begin{array}{c}\text { Resort RV Park } \\
\text { Water System - } 9\end{array}$ & $\begin{array}{l}\text { Surface } \\
\text { (creek) }\end{array}$ & 20 & 50 & Chlorination & $\begin{array}{l}\text { Water supplier lacking } \\
\text { knowledge on water } \\
\text { system [brought into } \\
\text { radar of health } \\
\text { authority via an E.coli } \\
\text { positive water sample] }\end{array}$ & $\begin{array}{l}\text { Meetings with engineers to } \\
\text { develop operation and } \\
\text { maintenance and monitoring } \\
\text { plans; BWA removal pending } \\
\text { negative water sample results and } \\
\text { plan approvals }\end{array}$ & $\begin{array}{l}\text { A } 15 \text { year old strata water system owned } \\
\text { by an Alberta-based business. Local } \\
\text { resident caretaker presumes } \\
\text { responsibilities of water supplier. } \\
\text { System serves } 6 \text { homes, } 14 \text { mobile } \\
\text { homes and RVs in summer; campground } \\
\text { opens seasonally during which } \\
\text { population served can go up to } 150 \text {. }\end{array}$ \\
\hline & & $\begin{array}{l}\text { Strata Water } \\
\text { System - } 10\end{array}$ & Deep well & 15 & 60 & $\begin{array}{l}\text { Chlorination, } \\
\text { Filtration, UV }\end{array}$ & $\begin{array}{l}\text { No water supplier } \\
\text { onsite to maintain } \\
\text { treatment and sample } \\
\text { during off-season }\end{array}$ & $\begin{array}{l}\text { Prior to opening for the season, } \\
\text { site was inspected, system flushed } \\
\text { and sampling resumed; BWA } \\
\text { removal pending negative water } \\
\text { sample results }\end{array}$ & $\begin{array}{l}\text { Opened in } 2007 \text {. Strata water system } \\
\text { managed by a property management } \\
\text { company; water supplier is a contracted } \\
\text { operator. System serves } 15 \text { vacation } \\
\text { homes; residents are onsite seasonally. }\end{array}$ \\
\hline & & Water System - 11 & Deep well & 111 & 300 & None & $\begin{array}{l}\text { Presence of fecal } \\
\text { coliform and low } \\
\text { numbers of total } \\
\text { coliform }\end{array}$ & $\begin{array}{l}\text { Reviewed water supplier's } \\
\text { sampling procedure to rule out } \\
\text { possibility of sampling } \\
\text { contamination. Leak detection } \\
\text { tests performed and illegal } \\
\text { connections repaired. BWA } \\
\text { removal pending negative water } \\
\text { sample results }\end{array}$ & $\begin{array}{l}\text { Established in 1919; updated 3-4 times } \\
\text { since. System was taken over by } \\
\text { regional district in 2000. Small town } \\
\text { serving residents, resort visitors and } \\
\text { water skiers. System has history of } \\
\text { frequent BWAs }\end{array}$ \\
\hline & \multirow{3}{*}{ 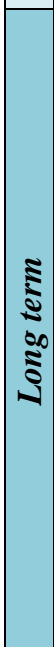 } & $\begin{array}{c}\text { General Store } \\
\text { Water System - } \\
12\end{array}$ & Deep well & 2 & 10 & None & $\begin{array}{l}\text { Source contaminated } \\
\text { with fecal coliform }\end{array}$ & $\begin{array}{l}\text { Past attempts to drill new, deeper } \\
\text { well failed to rid contamination; } \\
\text { now considering installing water } \\
\text { treatment }\end{array}$ & $\begin{array}{l}\text { System identified in 2003. Water } \\
\text { supplier is sole-proprietor of business } \\
\text { and homeowner. System serves his } \\
\text { home (household members, tenants) and } \\
\text { store (staff, customers who use } \\
\text { washroom and outdoor tap) }\end{array}$ \\
\hline & & $\begin{array}{l}\text { Mobile Home } \\
\text { Park Water } \\
\text { System - } 13\end{array}$ & $\begin{array}{l}\text { Surface } \\
\text { (creek) }\end{array}$ & $<15$ & 20 & None & $\begin{array}{l}\text { Presence of total and } \\
\text { fecal coliform in } \\
\text { water; no disinfection } \\
\text { at system }\end{array}$ & $\begin{array}{l}\text { No actions taken other than site } \\
\text { inspections and yearly reminder } \\
\text { letters to water system to } \\
\text { strategise and set goals towards } \\
\text { BWA removal. Health authority } \\
\text { requires staged improvements to } \\
\text { system beginning with } \\
\text { introduction of disinfection }\end{array}$ & $\begin{array}{l}\text { Discovered in } 1995 \text {; owned and } \\
\text { operated by water supplier and spouse. } \\
\text { System serves } 8 \text { trailers and } 1 \text { home. } \\
\text { Population served varies depending on } \\
\text { occupancy. }\end{array}$ \\
\hline & & $\begin{array}{c}\text { Campground } \\
\text { Water System - } 14\end{array}$ & Deep well & 1 & No data & None & $\begin{array}{l}\text { No maintenance or } \\
\text { testing program in } \\
\text { place; hand pump is of } \\
\text { unknown design }\end{array}$ & $\begin{array}{l}\text { Water supplier has submitted new } \\
\text { hand pump design for approval } \\
\text { by health authority engineers; } \\
\text { development and approval of } \\
\text { maintenance and monitoring } \\
\text { plans to follow }\end{array}$ & $\begin{array}{l}\text { Built in } 1964 \text {. Water system consists of } \\
\text { well connected to hand pump and spout. } \\
\text { Population served is transient as it } \\
\text { consists of park attendees. Water } \\
\text { supplier contracted by BC Parks }\end{array}$ \\
\hline
\end{tabular}




\begin{tabular}{|c|c|c|c|c|c|c|c|c|}
\hline & $\begin{array}{c}\text { Water System } \\
\text { Name }\end{array}$ & Source $^{1}$ & $\begin{array}{c}\# \\
\text { Connections }\end{array}$ & $\begin{array}{l}\text { \# Pop. } \\
\text { Served }\end{array}$ & $\begin{array}{c}\text { Central } \\
\text { Treatment }\end{array}$ & $\begin{array}{l}\text { Reason to } \\
\text { Issue }\end{array}$ & Actions & About the Water System \\
\hline \multirow{2}{*}{$\overbrace{\substack{2 \\
2}}^{5}$} & $\begin{array}{l}\text { Community } \\
\text { Water System - } \\
15\end{array}$ & 2 Deep wells & 220 & 500 & $\begin{array}{l}\text { Aeration, } \\
\text { Clarification, } \\
\text { Filtration }\end{array}$ & $\begin{array}{l}\text { Presence of total } \\
\text { coliform }\end{array}$ & $\begin{array}{l}\text { Introduced and implemented } \\
\text { disinfection protocol } \\
\text { (chlorination); BWA removal } \\
\text { pending negative water sample } \\
\text { results }\end{array}$ & $\begin{array}{l}30 \text { year old system serving mostly residential } \\
\text { homes and a few tourist/commercial } \\
\text { enterprises. Water supplier works as part of a } \\
\text { management team and reports to elected council } \\
\text { and Mayor }\end{array}$ \\
\hline & $\begin{array}{c}\text { Water System - } \\
16\end{array}$ & Well & 5 & 100 & $\begin{array}{l}\text { Filtration, } \\
\text { Softening, } \\
\text { Chlorination }\end{array}$ & $\begin{array}{l}\text { Water samples } \\
\text { did not meet } \\
\text { microbiological } \\
\text { standards }\end{array}$ & $\begin{array}{l}\text { Distribution system was cleaned } \\
\text { and disinfected; BWA removal } \\
\text { pending negative water sample } \\
\text { results }\end{array}$ & $\begin{array}{l}\text { Water system upgraded in } 2006 \text {. Hired } \\
\text { maintenance staff manages water system } \\
\text { operations. System serves hotel, restaurant, an } \\
\text { oil and gas camp and lodge for industrial } \\
\text { workers. }\end{array}$ \\
\hline & $\begin{array}{l}\text { Lake Water } \\
\text { System - } 17\end{array}$ & Shallow well & 25 & 70 & Chlorination* & $\begin{array}{l}\text { Water samples } \\
\text { did not meet } \\
\text { microbiological } \\
\text { standards; no } \\
\text { treatment in place }\end{array}$ & $\begin{array}{l}\text { Chlorinator was installed; BWA } \\
\text { removal pending negative water } \\
\text { sample results }\end{array}$ & $\begin{array}{l}\text { System discovered in } 1995 \text {. Essentially a } \\
\text { collection of } 25 \text { homes near a lake sharing a } \\
\text { well. Informally organised management system. }\end{array}$ \\
\hline \multirow{4}{*}{$\frac{\sqrt{3}}{3}$} & $\begin{array}{l}\text { Lake Water } \\
\text { System - } 18\end{array}$ & Cistern & 1 & 5 & $\begin{array}{l}\text { Filtration, } \\
\text { Chlorination }\end{array}$ & $\begin{array}{l}\text { Water samples } \\
\text { did not meet } \\
\text { microbiological } \\
\text { standards }\end{array}$ & $\begin{array}{l}\text { Site was inspected and the } \\
\text { cistern was found to be } \\
\text { compromised. Cistern to be } \\
\text { replaced. Proposals for new } \\
\text { water system are being } \\
\text { discussed. }\end{array}$ & $\begin{array}{l}\text { Water is trucked in from a filtered lake source } \\
\text { and stored in a cistern which serves a welding } \\
\text { shop and its employees. Water system is } \\
\text { managed by a large company overseeing a } \\
\text { number of systems in the region }\end{array}$ \\
\hline & $\begin{array}{l}\text { Lake Water } \\
\text { System - } 19\end{array}$ & $\begin{array}{l}\text { Surface } \\
\text { (lake) }\end{array}$ & 8 & 100 & None & $\begin{array}{l}\text { Microbiologically } \\
\text { unsafe water; no } \\
\text { treatment or } \\
\text { disinfection in } \\
\text { place }\end{array}$ & $\begin{array}{l}\text { Ongoing discussions and } \\
\text { inquiries on options. To be } \\
\text { decided whether existing system } \\
\text { be improved or secure an } \\
\text { alternative source for water } \\
\end{array}$ & $\begin{array}{l}\text { Decades old water system serving a mill site } \\
\text { and its employees. Source water is very poor } \\
\text { quality and has never been potable. A company } \\
\text { owns water system and has hired operators to } \\
\text { manage day to day system operations }\end{array}$ \\
\hline & $\begin{array}{c}\text { Abattoir Water } \\
\text { System - } 20\end{array}$ & $\begin{array}{l}\text { Deep well or } \\
\text { Surface? }\end{array}$ & $<10$ & 15 & None & $\begin{array}{l}\text { Presence of } \\
\text { coliform }\end{array}$ & $\begin{array}{l}\text { System cleaned and disinfected. } \\
\text { Health authority has asked for } \\
\text { information about the water } \\
\text { system to decide on solutions. } \\
\text { Water supplier suspects health } \\
\text { authority wants water treatment } \\
\text { installed. }\end{array}$ & $\begin{array}{l}19 \text { year old water system primarily serving } 3 \\
\text { homes, a dairy farm, a meat cut-wrap facility } \\
\text { and fire hydrants. Public being served } \\
\text { encompasses farm/business owner (the water } \\
\text { supplier), his family and staff. Brought into } \\
\text { radar of health authority when owner opened } \\
\text { cut-wrap facility in } 2009 \text {; was not under } \\
\text { government surveillance prior }\end{array}$ \\
\hline & $\begin{array}{l}\text { Camp Water } \\
\text { System - } 21\end{array}$ & $\begin{array}{c}\text { Surface } \\
\text { (lake) }\end{array}$ & 10 & 150 & $\begin{array}{l}\text { Filtration, } \\
\text { Chlorination, } \\
\text { UV* }\end{array}$ & $\begin{array}{l}\text { Microbiologically } \\
\text { unsafe water; no } \\
\text { water treatment } \\
\text { in place }\end{array}$ & $\begin{array}{l}\text { Health authority required } \\
\text { installation of a new treatment } \\
\text { system. Brand new water } \\
\text { system installed; BWA removal } \\
\text { pending negative water sample } \\
\text { results }\end{array}$ & $\begin{array}{l}\text { Water system serves a camp site for kids. Open } \\
\text { seasonally and population served varies } \\
\text { depending on number of camp attendees. Board } \\
\text { of members run camp and have delegated a } \\
\text { caretaker for managing water system } \\
\text { operations. Prior to new treatment system, } \\
\text { served raw water directly from lake. }\end{array}$ \\
\hline & $\begin{array}{l}\text { Lake Water } \\
\text { System - } 22\end{array}$ & Deep well & 10 & 300 & Chlorination & $\begin{array}{l}\text { Continuing } \\
\text { presence of } \\
\text { coliform in water } \\
\text { samples }\end{array}$ & $\begin{array}{l}\text { Corrected any equipment } \\
\text { malfunctions; improved } \\
\text { chlorinator. BWA removal } \\
\text { pending negative water sample } \\
\text { results }\end{array}$ & $\begin{array}{l}1.5 \text { year old water system serving a seasonally } \\
\text { open BC Provincial Park and camp site. } \\
\text { Population largely served are transient park } \\
\text { visitors and some staff members. Onsite Parks } \\
\text { Officer overlooks system management }\end{array}$ \\
\hline
\end{tabular}




\begin{tabular}{|c|c|c|c|c|c|c|c|c|c|}
\hline & & $\begin{array}{c}\text { Water System } \\
\text { Name }\end{array}$ & Source $^{1}$ & $\begin{array}{c}\# \\
\text { Connections }\end{array}$ & $\begin{array}{l}\text { \# Pop. } \\
\text { Served }\end{array}$ & $\begin{array}{c}\text { Central } \\
\text { Treatment }\end{array}$ & $\begin{array}{l}\text { Reason to } \\
\text { Issue }\end{array}$ & Actions & About the Water System \\
\hline & & $\begin{array}{l}\text { Community } \\
\text { Water System - } \\
23\end{array}$ & $\begin{array}{l}\text { Surface } \\
\text { (spring) }\end{array}$ & 7 & 50 & None** & $\begin{array}{l}\text { Presence of total } \\
\text { coliform in water } \\
\text { samples }\end{array}$ & $\begin{array}{l}\text { No action taken other than site } \\
\text { inspection. Installation of water } \\
\text { treatment at source is required } \\
\text { for BWA removal. }\end{array}$ & $\begin{array}{l}20 \text { year old water system serving a "good } \\
\text { neighbourhood" community of } 6 \text { homes, a } \\
\text { school (which has point of entry treatment } \\
\text { installed), and a church. Informal governance } \\
\text { system, where all members are deemed } \\
\text { responsible for water system. }\end{array}$ \\
\hline \multirow{6}{*}{ 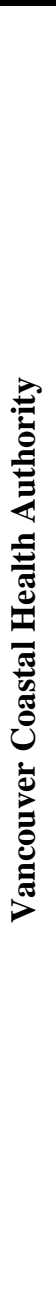 } & \multirow{3}{*}{$\frac{\sqrt[n]{2}}{5}$} & $\begin{array}{l}\text { Camp Water } \\
\text { System - } 24\end{array}$ & Shallow well & 12 & 175 & Chlorination & $\begin{array}{l}\text { Presence of total } \\
\text { coliform } \\
\text { indicating } \\
\text { disinfection } \\
\text { failure }\end{array}$ & $\begin{array}{l}\text { Disinfection equipment was } \\
\text { repaired and chlorine residuals } \\
\text { closely monitored. BWA } \\
\text { removal pending negative water } \\
\text { sample results }\end{array}$ & $\begin{array}{l}\text { Decades old water system owned by a society } \\
\text { and managed by onsite operator as part of } \\
\text { newly appointed board-based governance } \\
\text { structure. System serves } 3 \text { homes and a } \\
\text { seasonally running camp site. Public served } \\
\text { includes resident staff and camp guests. }\end{array}$ \\
\hline & & $\begin{array}{l}\text { Rehabilitation } \\
\text { Centre Water } \\
\text { System - } 25\end{array}$ & $\begin{array}{l}1 \text { Shallow } \\
\text { well, } 1 \text { Deep } \\
\text { well }\end{array}$ & 6 & 25 & None & $\begin{array}{l}\text { Presence of high } \\
\text { total coliform } \\
\text { counts }\end{array}$ & $\begin{array}{l}\text { Permit to install chlorination } \\
\text { system has been submitted by } \\
\text { water supplier for review by } \\
\text { health authority. BWA removal } \\
\text { upon installation of chlorinator } \\
\text { and demonstration of } \\
\text { satisfactory water quality }\end{array}$ & $\begin{array}{l}\text { Water system serves newly developed drug and } \\
\text { alcohol rehabilitation centre and its residents } \\
\text { and staff. Consultant to centre owner overlooks } \\
\text { business operations, including water system } \\
\text { indirectly via certified onsite water operator. }\end{array}$ \\
\hline & & $\begin{array}{l}\text { Water Works } \\
\text { System - } 26\end{array}$ & Deep well & 6 & 20 & None & $\begin{array}{l}\text { Presence of total } \\
\text { coliform }\end{array}$ & $\begin{array}{l}\text { System was flushed and water } \\
\text { re-sampled. Discussions of } \\
\text { introducing disinfection via } \\
\text { super-chlorination at water } \\
\text { system. BWA removal pending } \\
\text { negative water sample results }\end{array}$ & $\begin{array}{l}\text { Old water system (records dating as far back as } \\
\text { 1980) with sole proprietor and single owner as } \\
\text { water supplier and the only one managing water } \\
\text { system operations. }\end{array}$ \\
\hline & \multirow{3}{*}{$\frac{\sqrt{3}}{30}$} & $\begin{array}{c}\text { Strata } \\
\text { Association } \\
\text { Water System - } \\
27\end{array}$ & Shallow well & 5 & 10 & None** & $\begin{array}{l}\text { Consistently poor } \\
\text { bacteriological } \\
\text { quality of water; } \\
\text { [Was issued as } \\
\text { part of a blanket } \\
\text { advisory in } \\
\text { region] } \\
\end{array}$ & $\begin{array}{l}\text { Water system upgraded as per } \\
\text { health authority } \\
\text { recommendations. BWA } \\
\text { removal upon each house } \\
\text { installing a point-of-entry } \\
\text { device and demonstration of } \\
\text { satisfactory water quality }\end{array}$ & $\begin{array}{l}40 \text { year old water system on small strata lot, } \\
\text { shared by } 8 \text { homes and jointly managed by an } \\
\text { elected strata council. }\end{array}$ \\
\hline & & $\begin{array}{l}\text { Resort Water } \\
\text { System - } 28\end{array}$ & $\begin{array}{l}\text { Surface } \\
\text { (creek) }\end{array}$ & 1 & 40 & None & $\begin{array}{l}\text { Untreated surface } \\
\text { water }\end{array}$ & $\begin{array}{l}\text { Conducted water analysis on } \\
\text { source to determine water } \\
\text { treatment options. Health } \\
\text { authority requires staging } \\
\text { upgrades to the water system, } \\
\text { starting with microbiological } \\
\text { control measures. }\end{array}$ & $\begin{array}{l}30 \text { year old water system serving a seasonally } \\
\text { operating resort. Population served is primarily } \\
\text { resident staff and guests who come for } \\
\text { boating/fishing. Business is suffering } \\
\text { financially and water supplier considering } \\
\text { closing business or filing for bankruptcy }\end{array}$ \\
\hline & & $\begin{array}{l}\text { Sportsman's Club } \\
\text { Water System - } \\
29\end{array}$ & $\begin{array}{l}\text { Surface } \\
\text { (lake) }\end{array}$ & 1 & 40 & None & $\begin{array}{l}\text { Untreated surface } \\
\text { water }\end{array}$ & $\begin{array}{l}\text { Conducted water analysis on } \\
\text { source to determine water } \\
\text { treatment options. Health } \\
\text { authority requires staging } \\
\text { upgrades to the water system, } \\
\text { starting with microbiological } \\
\text { control measures. }\end{array}$ & $\begin{array}{l}\text { Similar to Resort Water System-28, the system } \\
\text { serves a seasonally operating lodge. Population } \\
\text { served is primarily resident staff and tourists. } \\
\text { Business is suffering financially and water } \\
\text { supplier (owner) considering closing business } \\
\text { or filing for bankruptcy }\end{array}$ \\
\hline
\end{tabular}




\begin{tabular}{|c|c|c|c|c|c|c|c|}
\hline $\begin{array}{c}\text { Water System } \\
\text { Name }\end{array}$ & Source $^{1}$ & $\begin{array}{c}\# \\
\text { Connections }\end{array}$ & $\begin{array}{l}\text { \# Pop. } \\
\text { Served }\end{array}$ & $\begin{array}{c}\text { Central } \\
\text { Treatment }\end{array}$ & $\begin{array}{l}\text { Reason to } \\
\text { Issue }\end{array}$ & Actions & About the Water System \\
\hline $\begin{array}{c}\text { Club Office } \\
\text { Water System - } \\
30\end{array}$ & Deep well & $2-10$ & 15 & None & $\begin{array}{l}\text { Presence of } \\
\text { E.coli and total } \\
\text { coliform }\end{array}$ & $\begin{array}{l}\text { Well was chlorinated. Health } \\
\text { authority expressed need for the } \\
\text { initiation of regular water } \\
\text { sampling by water supplier. } \\
\text { BWA removal pending negative } \\
\text { water sample results }\end{array}$ & $\begin{array}{l}\text { Water system serves water to an outstation } \\
\text { facility/office where campers (kids) are } \\
\text { temporarily held before being transported to a } \\
\text { camp site. Public served is primarily campers } \\
\text { and staff. Hired caretaker manages water } \\
\text { system operations. }\end{array}$ \\
\hline $\begin{array}{c}\text { Water User's } \\
\text { Community } \\
\text { Water System - } \\
31\end{array}$ & $\begin{array}{l}\text { Surface } \\
\text { (creek) }\end{array}$ & 14 & 30 & None & $\begin{array}{l}\text { Inconsistent } \\
\text { presence of total } \\
\text { coliform; no } \\
\text { water treatment } \\
\text { in place }\end{array}$ & $\begin{array}{l}\text { Water treatment options were } \\
\text { considered and plans for point- } \\
\text { of-use devices were submitted } \\
\text { to health authority for approval. } \\
\text { BWA removal pending } \\
\text { installation of devices in each } \\
\text { house and demonstration of } \\
\text { satisfactory water quality }\end{array}$ & $\begin{array}{l}60 \text { year old water system serving a small } \\
\text { community of neighbours ( } 10 \text { homes) and is } \\
\text { owned and managed by the water supplier. }\end{array}$ \\
\hline
\end{tabular}

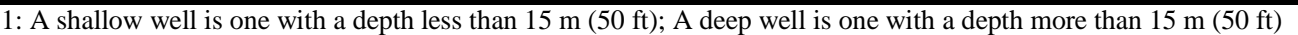

* Prior to the BWA's issue, there was no central water treatment at the system. Some form of treatment or disinfection had been installed by the time of the interview.

** No central treatment at water system, but water was being treated via a point -of-use or point-of-entry device, by at least one unit/household in the community, at the time of interview 
Table 5 summarizes water system characteristics in three ways: as reported by health authority officials for all 31 water systems; as reported by health authority officials for the subset of 14 water systems for which water supplier interviews were also completed; and as reported by water suppliers for the subset of 14 water systems about which they were interviewed. This reporting style is followed elsewhere in this report, and allows information about all water systems to be shown, as well as comparisons between responses of health authority officials and water suppliers on the same systems. The majority of the water systems investigated served 50 persons or fewer. Among the set of water systems investigated, 8 (26\%) operated only seasonally, and $16(52 \%)$ operated and served drinking water all year round; this information was unavailable for the remaining 7 water systems. For seasonally operating water systems and water systems serving transient populations, the numbers of people served drinking water varied depending on the time of year. The table reports population sizes at maximum capacity.

Among all 31 water systems, the size of water system (number of connections and population served) did not differ between long-term and short-term BWAs. Interestingly and counter-intuitively, the majority of the BWAs were on water systems deriving water from a groundwater source (58\%), and specifically deep wells (45\%). It is unclear why this was the case. It should be noted that the majority of short-term BWAs were on a groundwater source $(85 \%)$, whereas $39 \%$ of long-term BWAs were on a water system on groundwater source and $50 \%$ of them on surface water. There was a statistically significant difference between BWA category (short-term, long-term) and whether the source was surface, ground or other (Chisquare $=6.71, \mathrm{df}=2, \mathrm{p}$-value $=0.035)$. When comparing specific types of water sources, $69 \%$ of short-term BWAs were at water systems on a deep well water source versus $28 \%$ of long-term BWAs. This difference was also statistically significant (Chi-square $=4.69, \mathrm{df}=1, \mathrm{p}$-value $=$ 0.03). A greater proportion of long-term BWAs were on systems on surface water $(50 \%)$ than short-term $(15 \%)$, but this difference was not significant (Chi-square $=2.58, \mathrm{df}=1, \mathrm{p}$-value $=$ $0.11)$.

Nine of the 31 water systems had some form of barrier or safeguard in place at the source of the water, such as a trench in between the lake and well to allow for crude infiltration, the implementation of a source water protection plan or fencing off the source to prevent human and wildlife access. Of the water systems reported to have some barrier or safeguard in place, 8 of 
the 9 were on a groundwater source. A greater proportion of water systems that had some safeguard to the source water were on a short-term BWA (46\%) rather than a long-term one (17\%), but this difference was not statistically significant.

As shown in Table 5, close to half of the water systems had some form of treatment in place. Please note, as a clarification and for the purpose of this thesis, treatment may refer to any physical or chemical processing of the water. This may include anything from simple disinfection procedures to more sophisticated processes such as filtration. Ten of the 14 systems with treatment had it at the system level, whereas 4 had treatment at the tap via point-of-use or point-of-entry devices. Chlorination and filtration were the most common forms of water disinfection and treatment. None of the systems implemented ozone, although this form of disinfection was mentioned by a number of participants as possibly being used in the future. Most of the systems on a short-term BWA had treatment (62\%) but this was not the case for long-term BWAs (33\%); this difference was not statistically significant.

Seven water systems had barriers or safeguards in place at the level of distribution. These included such measures as the maintenance and monitoring of a secondary disinfection (chlorine) residual, back-flow protection and cross-connection control programs. Of those systems with such barriers in place, the majority (86\%) were under a short-term BWA as opposed to a longterm BWA (14\%). This difference was statistically significant (Chi-square $=4.98, \mathrm{df}=1, \mathrm{p}$ value $=0.026)$.

The overall level of agreement between health authority officials and water suppliers, as measured by the Kappa statistic (Table 5) is fairly strong. Except for the question on the presence of protective barriers at the water source, there was at least $50 \%$ agreement beyond chance in all responses from water suppliers and health authority officials on factual questions based on the water system's characteristics. It is interesting to note that although the same proportion of health authority officials and water suppliers reported that the water supplier was trained - making it seem like there was complete agreement between the two groups - this was actually not the case and the crude percent agreement between them was $71 \%$ agreement. 
Other notable inconsistencies between health authority officials and water suppliers' responses were found on the type of water source and on the presence of water treatment. In one case, the health authority official reported that the water source was a deep well, whereas the water supplier reported the source to be a spring (surface) source. In this case, the health authority official may have been incorrect, as he expressed that not much was known about this system. In 3 cases, the water supplier and the health authority official gave opposing responses on whether there was water treatment at the water system. It is very likely that this may be because by the time the water supplier was interviewed (which at times was months after the health authority official was interviewed), some form of water treatment or disinfection had been introduced at the water system.

With regards to the date the BWA was issued, only 6 of the 14 health authority officials and water suppliers provided the same date. Two health authority officials and 3 water suppliers did not know when the BWA was issued nor had any record of the date. On a related note, 6 health authority officials (19\%) among all 31, and 6 of the 14 water suppliers interviewed (43\%) provided a BWA issue date that was different than that posted online on the health authority's website. 
Table 5: Characteristics of all 31 randomly selected water systems under BWA, and on the subset of 14 systems with reported information from both health authority officials and water suppliers. Percent agreement and kappas for agreement beyond chance between health authority officials and water suppliers on information reported on the subset of 14 water systems.

\begin{tabular}{|c|c|c|c|c|c|}
\hline \multirow[t]{2}{*}{ (20) } & \multicolumn{3}{|c|}{ \# Of water systems (\%) } & \multirow[b]{2}{*}{$\begin{array}{c}\% \\
\text { Agreement }\end{array}$} & \multirow[b]{2}{*}{$\begin{array}{c}\text { Kappa } \\
\text { Statistic** }\end{array}$} \\
\hline & $\begin{array}{l}\text { As reported by } \\
\text { health authority } \\
\text { officials } \\
\text { (31 water } \\
\text { systems) }\end{array}$ & $\begin{array}{l}\text { As reported by } \\
\text { health authority } \\
\text { officials } \\
\text { (14 water } \\
\text { systems) }\end{array}$ & $\begin{array}{l}\text { As reported } \\
\text { by water } \\
\text { suppliers } \\
\text { (14 water } \\
\text { systems) }\end{array}$ & & \\
\hline \multicolumn{4}{|l|}{ Is the water supplier trained? } & \multirow{3}{*}{$71 \%$} & \multirow{3}{*}{0.43} \\
\hline - Yes & $10(32 \%)$ & $7(50 \%)$ & $7(50 \%)$ & & \\
\hline - No & $19(61 \%)$ & $7(50 \%)$ & $7(50 \%)$ & & \\
\hline \multicolumn{4}{|l|}{$\begin{array}{l}\text { The number of active } \\
\text { connections: }\end{array}$} & \multirow{4}{*}{$86 \%$} & \multirow{4}{*}{0.65} \\
\hline - 2-14 & $23(74 \%)$ & $10(71 \%)$ & $10(71 \%)$ & & \\
\hline - $15-300$ & $8(26 \%)$ & $4(29 \%)$ & $4(29 \%)$ & & \\
\hline - $301+$ & $0(0 \%)$ & $0(0 \%)$ & $0(0 \%)$ & & \\
\hline \multicolumn{4}{|l|}{ Population served: } & \multirow{5}{*}{$69 \%$} & \multirow{5}{*}{0.57} \\
\hline - $1-10$ persons & $9(29 \%)$ & $3(21 \%)$ & $1(7 \%)$ & & \\
\hline - 11-50 persons & $10(32 \%)$ & $4(29 \%)$ & $6(43 \%)$ & & \\
\hline - 51-100 persons & $5(16 \%)$ & $2(14 \%)$ & $2(14 \%)$ & & \\
\hline - 101-500 persons & $6(19 \%)$ & $4(29 \%)$ & $5(36 \%)$ & & \\
\hline \multicolumn{4}{|l|}{ Water source: } & \multirow{3}{*}{$92 \%$} & \multirow{3}{*}{0.81} \\
\hline - Surface water & $11(35 \%)$ & $3(21 \%)$ & $4(29 \%)$ & & \\
\hline - Ground water & $18(58 \%)$ & $10(71 \%)$ & $9(64 \%)$ & & \\
\hline - Shallow well ${ }^{1}$ & $5(16 \%)$ & $4(29 \%)$ & $3(21 \%)$ & $92 \%$ & 0.81 \\
\hline - Deep well ${ }^{2}$ & $14(45 \%)$ & $7(50 \%)$ & $5(36 \%)$ & $86 \%$ & 0.70 \\
\hline - Well (unknown depth) & $1(3 \%)$ & $1(7 \%)$ & $1(7 \%)$ & $100 \%$ & 1.00 \\
\hline - Mixed sources & $1(3 \%)$ & $1(7 \%)$ & $1(7 \%)$ & $100 \%$ & 1.00 \\
\hline - Cistern & $1(3 \%)$ & $0(0 \%)$ & $0(0 \%)$ & $100 \%$ & $\mathrm{n} / \mathrm{a}$ \\
\hline \multicolumn{4}{|l|}{$\begin{array}{l}\text { Are there barriers/safeguards at } \\
\text { source? }\end{array}$} & \multirow{3}{*}{$43 \%$} & \multirow{3}{*}{-0.14} \\
\hline - Yes & $9(29 \%)$ & $7(50 \%)$ & $13(93 \%)$ & & \\
\hline • No & $22(71 \%)$ & $7(50 \%)$ & $1(7 \%)$ & & \\
\hline \multicolumn{4}{|l|}{$\begin{array}{l}\text { Is there any water treatment } \\
\text { and/or disinfection in place? }\end{array}$} & \multirow[b]{2}{*}{$71 \%$} & \multirow[b]{2}{*}{0.43} \\
\hline - Yes & $14(45 \%)$ & $7(50 \%)$ & $9(64 \%)$ & & \\
\hline - Chlorination ${ }^{3,4}$ & $9 / 14(64 \%)$ & $4 / 7(57 \%)$ & $5 / 9(56 \%)$ & $83 \%$ & 0.64 \\
\hline - Filtration ${ }^{3,4}$ & $5 / 14(36 \%)$ & $3 / 7(43 \%)$ & $3 / 9(33 \%)$ & $92 \%$ & 0.75 \\
\hline - Ultra-violet ${ }^{3,4}$ & $2 / 14(14 \%)$ & $1 / 7(14 \%)$ & $3 / 9(33 \%)$ & $83 \%$ & 0.43 \\
\hline - Ozone $e^{3,4}$ & $0 / 14(0 \%)$ & $0 / 7(0 \%)$ & $0 / 9(0 \%)$ & $100 \%$ & $\mathrm{n} / \mathrm{a}$ \\
\hline $\begin{array}{l}\text { - Point of use/Point of } \\
\text { entry device } \\
\text { ent, }^{3}\end{array}$ & $4 / 14(29 \%)$ & $2 / 7(29 \%)$ & $1 / 9(11 \%)$ & $100 \%{ }^{6}$ & $1.00^{6}$ \\
\hline - No & $17(55 \%)$ & $7(50 \%)$ & $5(36 \%)$ & $71 \%$ & 0.43 \\
\hline \multicolumn{4}{|l|}{$\begin{array}{l}\text { Are there barriers/safeguards at } \\
\text { distribution level? }\end{array}$} & \multirow{3}{*}{$69 \%$} & \multirow{3}{*}{0.40} \\
\hline - Yes & $7(23 \%)$ & $5(36 \%)$ & $7(50 \%)$ & & \\
\hline - $\mathrm{No}$ & $24(77 \%)$ & $9(64 \%)$ & $6(43 \%)$ & & \\
\hline
\end{tabular}

1: A well with a depth less than $15 \mathrm{~m}(50 \mathrm{ft})$

2: A well with a depth more than $15 \mathrm{~m}(50 \mathrm{ft})$

3: Proportions and percentages calculated with the denominator as the number of participants who answered "yes" to water treatment/disinfection

4: Treatment at the central/water system-level 
5: Such devices usually incorporate filtration and UV light disinfection as the means for water treatment

6: The Kappa test removes all unpaired data from analysis (such as those where only the health authority answered and not the water supplier and vice versa). As a result, the remaining data set of pairs of health authority and water supplier responses included only those where both groups were in full agreement, hence a percent agreement of $100 \%$ and a perfect Kappa score.

* A Kappa statistic equal to 1 indicates perfect agreement and equal to 0 indicates agreement due to chance (46)

\subsubsection{What's in a name? - A clarification of terminologies and semantics}

To get some understanding of the terminology being used among health authority officials and water suppliers, participants were asked which types of public notification(s) are used by their health authority (or water systems) and the definition of the notification(s). Box 1 summarizes this information. The terms "boil water notices" (74\%), "water quality advisories" (84\%) and "do not drink advisories" (45\%) were the most common among health authority officials. The same was true for water suppliers, except that the term "boil water advisories" (79\%) was more commonly used than "boil water notices" (29\%).

While many of the health authority officials provided 'text-book', almost theoretical definitions of the types of public notifications and when they are issued - largely referenced from the Drinking Water Officer's Guide - the water suppliers' awareness and knowledge on this topic was relatively limited and largely dependent on and reflective of their personal and practical experiences at the water system. Table 6 provides information on the numbers of health authority officials and water suppliers who correctly identified and defined the three main types of public notifications, as per the Drinking Water Officer's Guide. Many water suppliers provided examples of when a particular type of public notification would be issued, rather than a definition of it; nearly all examples were reflective of the BWA on their water system. All but one water supplier identified that BWAs are issued because there is "something" in the water that renders it potentially unsafe to drink. Reasons for when to issue a BWA included "when the lab reports on the water samples indicate so", "when there is high total coliform" or "when the health authorities say so". The water suppliers' understanding of other types of public notifications in relation to BWAs was poor. Only two water suppliers correctly articulated the differences between BWAs, water quality advisories and do not drink advisories. Others simply stated that they did not know what the other notifications meant or when they would be issued and a few others saw no difference in BWAs and other notifications. This may be because many water suppliers had never encountered any type of public notification, other than BWAs, despite having heard of them. 
Box 1: Terminology for public notifications of drinking water quality used by health authority officials

\begin{tabular}{|c|c|c|c|}
\hline & Boil water notices & Water quality advisories & Do not drink advisories \\
\hline & Boil water advisories & Drinking water advisories & Do not use water notices \\
\hline & Boil water orders & Water quality notifications & Do not use \\
\hline $\begin{array}{c}\text { Least } \\
\text { Common }\end{array} \mid$ & & Drinking water notifications & Do not use advisories \\
\hline When to & $\begin{array}{l}\text { When there is a known } \\
\text { or potential threat to } \\
\text { drinking water that is } \\
\text { microbiological } \\
\text { (bacterial, viral or } \\
\text { parasitic) in nature. } \\
\text { The risk can be } \\
\text { adequately addressed by } \\
\text { boiling the water as a } \\
\text { short-term form of } \\
\text { treatment. }\end{array}$ & $\begin{array}{l}\text { When there is some level of } \\
\text { threat particularly affecting } \\
\text { a subset of the general } \\
\text { population (susceptible } \\
\text { individuals) and not } \\
\text { necessarily everyone; the } \\
\text { threat is not significant } \\
\text { enough to require a boil } \\
\text { water advisory or do not } \\
\text { drink advisory }\end{array}$ & $\begin{array}{l}\text { When there is a risk that } \\
\text { cannot be adequately } \\
\text { addressed by issuing a } \\
\text { water quality advisory or } \\
\text { by boiling the water (and } \\
\text { in some cases, boiling } \\
\text { would worsen the water } \\
\text { quality); the water is not } \\
\text { safe for domestic use. }\end{array}$ \\
\hline & $\begin{array}{l}\text { A boil water order is a } \\
\text { legal written order and } \\
\text { formalized version of a } \\
\text { boil water advisory } \\
\text { requiring water supplier } \\
\text { to operate under boil } \\
\text { water conditions. }\end{array}$ & & \\
\hline $\begin{array}{c}\text { Common } \\
\text { Reasons to } \\
\text { Issue: }\end{array}$ & $\begin{array}{l}\text { - Waterborne outbreak } \\
\text { conditions } \\
\text { - Bacteriological } \\
\text { quality of water is } \\
\text { below the accepted } \\
\text { standard (presence of } \\
\text { E.coli in water and/or } \\
\text { repeated trends of } \\
\text { total coliform) } \\
\text { - No treatment in place } \\
\text { at water system } \\
\text { - Water system } \\
\text { treatment breakdown } \\
\text { - Inadequate } \\
\text { disinfection at water } \\
\text { system } \\
\text { - High turbidity }\end{array}$ & $\begin{array}{l}\text { - Chemical exceedances } \\
\text { (such as high sodium or } \\
\text { high iron levels, which } \\
\text { may affect individuals } \\
\text { on a sodium-restricted } \\
\text { diet or infants, } \\
\text { respectively) } \\
\text { - Water is not aesthetically } \\
\text { pleasing (colour, odour) } \\
\text { - Medium-to-high turbidity }\end{array}$ & $\begin{array}{l}\text { - Chemical } \\
\text { contamination or } \\
\text { exceedances (such as } \\
\text { unacceptable levels of } \\
\text { naturally occurring } \\
\text { nitrates, lead or arsenic) } \\
\text { - Accidents - such as } \\
\text { chemical spills } \\
\text { - Acts of vandalism, } \\
\text { sabotage or } \\
\text { bioterrorism that may } \\
\text { affect water supply } \\
\text { - Natural disasters, such } \\
\text { as earthquakes or } \\
\text { mudslides } \\
\text { - For precautionary } \\
\text { purposes when there is } \\
\text { some unknown threat } \\
\text { that will require time to } \\
\text { determine }\end{array}$ \\
\hline
\end{tabular}


Table 6: The number of health authority officials and water suppliers who were at least partially correct in identifying and defining the three main types of public notifications, as per the Drinking Water Officer's Guide

\begin{tabular}{|c|c|c|c|}
\hline & Type of Public Notice and Definition & $\begin{array}{l}24^{*} \text { Health } \\
\text { Authority } \\
\text { Officials }(\%)\end{array}$ & $\begin{array}{l}14 \text { Water } \\
\text { Suppliers } \\
\text { (\%) }\end{array}$ \\
\hline $\begin{array}{l}\text { Boil } \\
\text { Water } \\
\text { Advisory }\end{array}$ & $\begin{array}{l}\text { When there is a known or potential threat to drinking } \\
\text { water that is microbiological (bacterial, viral or } \\
\text { parasitic) in nature. The risk can be adequately } \\
\text { addressed via boiling the water as a short-term form of } \\
\text { treatment. }\end{array}$ & $\begin{array}{c}24 \\
(100 \%)\end{array}$ & $\begin{array}{c}11 \\
(79 \%)\end{array}$ \\
\hline $\begin{array}{l}\text { Water } \\
\text { Quality } \\
\text { Advisory }\end{array}$ & $\begin{array}{l}\text { When there is some level of threat particularly } \\
\text { affecting a subset of the general population (susceptible } \\
\text { individuals) and not necessarily everyone; the threat is } \\
\text { not significant enough to require a boil water advisory } \\
\text { or do not drink advisory }\end{array}$ & $\begin{array}{c}21 \\
(87.5 \%)\end{array}$ & $\begin{array}{c}2 \\
(14 \%)\end{array}$ \\
\hline $\begin{array}{l}\text { Do Not } \\
\text { Drink } \\
\text { Advisory }\end{array}$ & $\begin{array}{l}\text { When there is a risk that cannot be adequately } \\
\text { addressed by issuing a water quality advisory or by } \\
\text { boiling the water (and in some cases, boiling would } \\
\text { worsen the water quality); the water is not safe for } \\
\text { domestic use. }\end{array}$ & $\begin{array}{c}22 \\
(92 \%)\end{array}$ & $\begin{array}{c}2 \\
(14 \%)\end{array}$ \\
\hline
\end{tabular}

* As 7 of the 31 health authority officials had participated in more than one interview, the data presented is based on responses from 24 different health authority officials.

In response to the known, mixed usage of the terms "boil water advisories" and "boil water notices" and the confusion with the purpose of "boil water orders", one health authority official had this to say:

"Practically we use the terms interchangeably but to be consistent, we try to move towards the term "boil water notice" to be consistent with [the] Drinking Water Officer's Guide. [The] challenge with that is, internationally and in other jurisdictions, "boil water advisory" is still called. [The] media will often times use "boil water advisory". [It's a] bit of a struggle to change well established labels to something new... [The] "boil water order" is a misnomer. You can't order someone to boil their water. We can only order a purveyor to issue a boil water advisory."

Are BWAs issued because water is unsafe to drink and a risk to health or when water quality is compromised and suboptimal? Figure 4 graphically illustrates how health authority officials and water suppliers answered this question. 


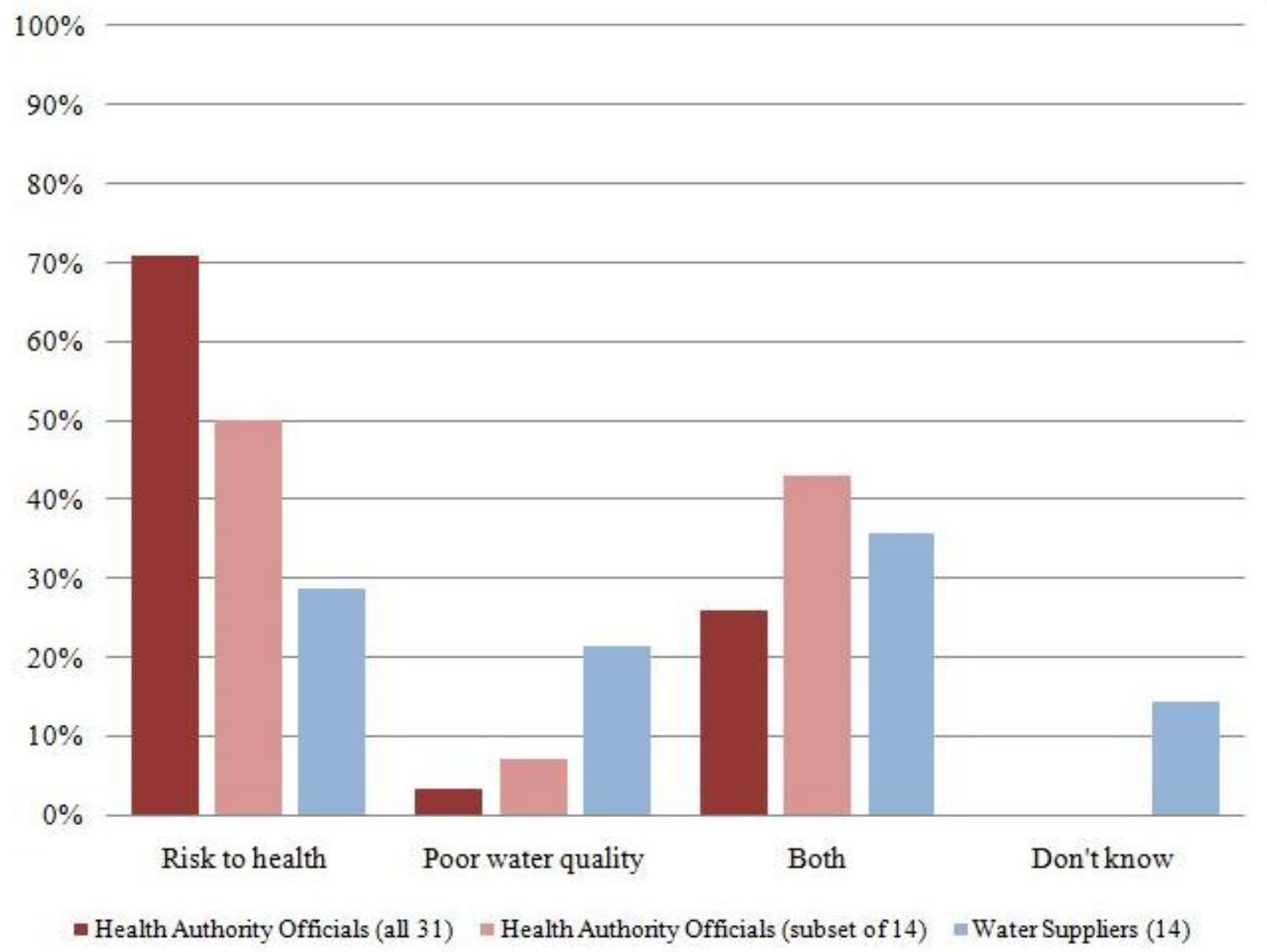

Figure 4: Why are boil water advisories primarily issued? A comparison of responses from health authority officials and water suppliers

\subsubsection{To issue or not to issue? That is the question}

Fifty-two percent of health authority officials and 57\% of water suppliers interviewed were not present at the time the BWA was issued. This may be because the BWA was issued long before the official or water supplier started work in their current position, the official or water supplier recently took-over management or oversight of the water system under BWA, or they were temporarily away from work or offsite at that time. The health authority officials, who were not present at the time of decision-making depended heavily on documented records to answer questions on the process behind the decision to issue. Some officials communicated with the health authority officials who had been the one who issued the BWA in preparation for the interview, whereas some took an educated guess as to what would have likely taken place at that time. 
Table 7 provides the reasons why the BWAs were in place in all 31 and in the subset of 14 water systems. The reasons listed are not mutually exclusive and at 9 water systems, more than one reason for issuing was identified. At 8 of these, the reason for issuing the BWA was in part due to positive bacteriological results.

Table 7: Reasons for issuing boil water advisories at all 31 water systems and at the 14 water systems with data from both health authority officials and water suppliers

\begin{tabular}{lccc}
\hline \multicolumn{1}{c}{ The primary reason for } & \multicolumn{1}{c}{$\begin{array}{c}\text { All 31 Water } \\
\text { Systems }\end{array}$} & \multicolumn{2}{c}{ Subset of 14 Water Systems } \\
\cline { 2 - 4 } $\begin{array}{l}\text { issuing the boil water advisory } \\
\text { \#ositive bacteriological water }\end{array}$ & $\begin{array}{c}\text { \# of systems as } \\
\text { reported by HA } \\
\text { officials }\end{array}$ & $\begin{array}{c}\text { \# of systems as } \\
\text { reported by HA } \\
\text { officials }\end{array}$ & $\begin{array}{c}\text { \# of systems as } \\
\text { reported by } \\
\text { Water Suppliers }\end{array}$ \\
$\begin{array}{l}25(81 \%) \\
\text { sample results }\end{array}$ & $9(29 \%)$ & $12(86 \%)$ & $10(71 \%)$ \\
$\begin{array}{l}\text { No treatment and/or } \\
\text { disinfection in place at the } \\
\text { water system }\end{array}$ & $2(6 \%)$ & $3(21 \%)$ & $1(7 \%)$ \\
$\begin{array}{l}\text { Lack of information about the } \\
\text { water system }\end{array}$ & $2(6 \%)$ & $1(7 \%)$ & $0(0 \%)$ \\
\hline $\begin{array}{l}\text { Unprotected or poor source } \\
\text { No sampling or maintenance } \\
\text { in place }\end{array}$ & $1(3 \%)$ & $2(14 \%)$ & $1(7 \%)$ \\
$\begin{array}{l}\text { Treatment and/or disinfection } \\
\text { failure }\end{array}$ & $1(3 \%)$ & $0(0 \%)$ & $2(14 \%)$ \\
\hline
\end{tabular}

There was at least partial agreement on the reason behind issuing of the BWA between 8 health authority officials and water suppliers. Three water suppliers mentioned that they were not completely sure why the BWA was in place, but did provide a reason based on their assumptions.

Table 8 compares the reasons why the BWAs were issued by BWA category (long-term versus short-term). Microbiologically unsafe water and treatment or disinfection failure were reasons common to short-term BWAs, the latter only being cited for short-term BWAs. A lack of water treatment or disinfection, a lack of information about the water system, and a lack of sampling or maintenance at the water system were only cited as reasons for long-term BWAs. These differences were not statistically significant $($ Chi-square $=10.4, \mathrm{df}=5, \mathrm{p}$-value $=0.065)$, but still provide insight into the differing system characteristics that become apparent for advisories that become long-term. 
Table 8: Reasons for issuing long-term versus short-term BWAs, as reported by 31 health authority officials

\begin{tabular}{|c|c|c|}
\hline \multirow[t]{2}{*}{ The primary reasons for issuing the boil water advisory } & \multicolumn{2}{|c|}{ \# of Water Systems (\%) } \\
\hline & $\begin{array}{l}\text { Long-Term } \\
\quad(n=18)\end{array}$ & $\begin{array}{l}\text { Short-Term } \\
\quad(n=13)\end{array}$ \\
\hline Positive bacteriological water sample results & $13(72 \%)$ & $12(92 \%)$ \\
\hline $\begin{array}{l}\text { No treatment and/or disinfection in place at the water } \\
\text { system }\end{array}$ & $9(50 \%)$ & $0(0 \%)$ \\
\hline Lack of information about the water system & $2(11 \%)$ & $0(0 \%)$ \\
\hline Unprotected or poor source & $1(6 \%)$ & $1(8 \%)$ \\
\hline No sampling or maintenance in place & $1(6 \%)$ & $0(0 \%)$ \\
\hline Treatment and/or disinfection failure & $0(0 \%)$ & $1(8 \%)$ \\
\hline
\end{tabular}

Figure 5 shows the criteria or factors that were considered by health authority officials and water suppliers when deciding whether to issue the BWA or not. In general, the decision to issue a BWA was made within the same day that the (potential or confirmed) risk to water quality became known.

According to the health authority officials, 19 of all 31 BWAs (61\%) were on systems where both the lack of water treatment and positive bacteriological water samples triggered the issuing of the BWA, that is, both were mentioned as either the reason for issuing or as a factor considered in the decision-making process. However, 5 of these water systems were reported to have some form of water treatment or disinfection in place at the time of interview. It is therefore possible (especially in the case of long-term BWAs) that the lack of water treatment was considered in the decision to issue a BWA at the time of issue, but at the time of interview, some type of water treatment and/or disinfection had been since introduced and was being used. The majority of systems that lacked treatment and had microbiologically unsafe water (12/19) were on long-term advisories. 


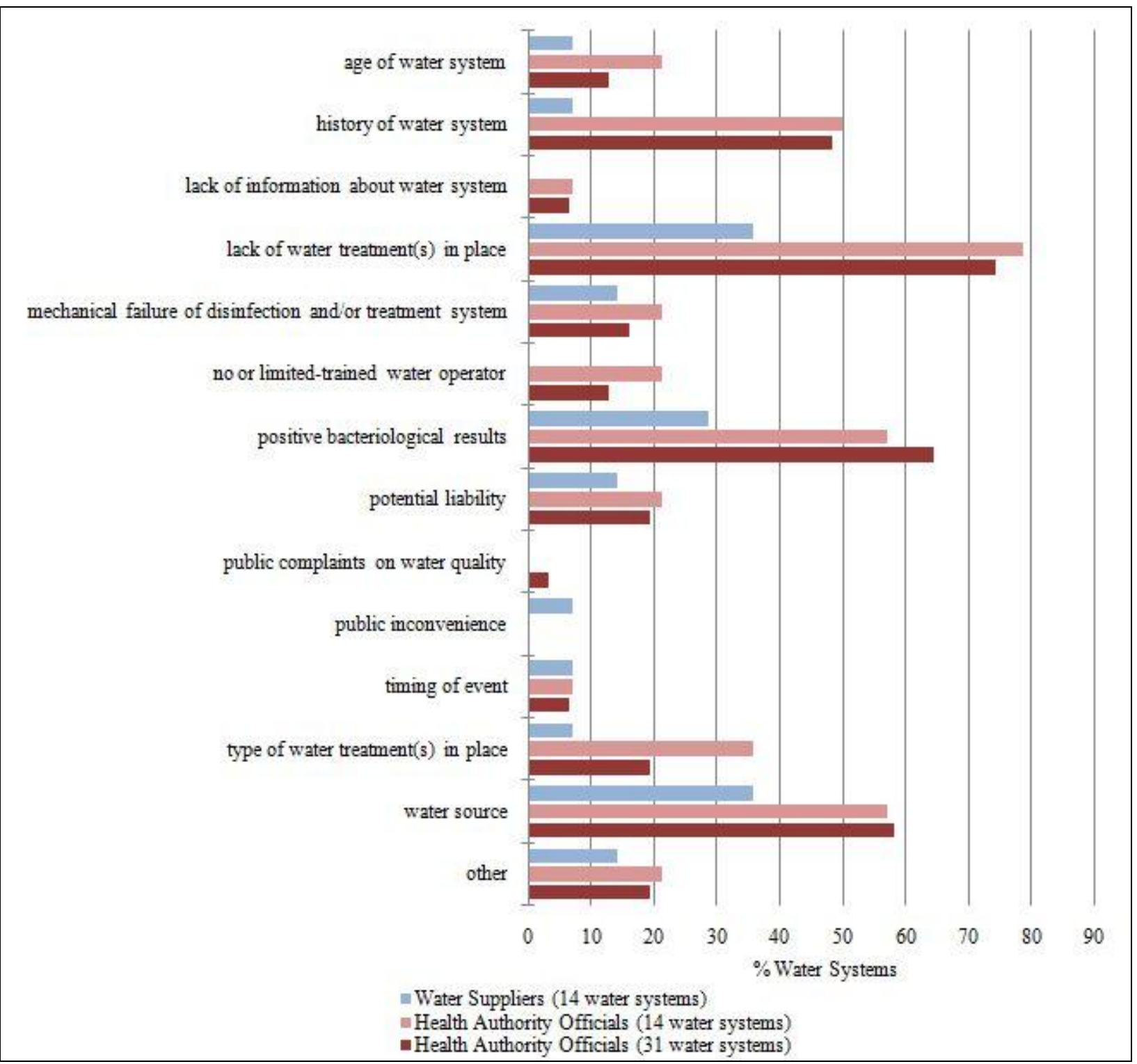

Figure 5: Decision-making criteria for issuing boil water advisories as reported by health authority officials and water suppliers

Table 9 lists the individuals who were involved in the decision-making process for issuing BWAs at the 31 water systems. Where more than one person was involved, most teams were made up by three individuals: a primary decision-maker, a consultant and a water system representative. In $77 \%$ of all 31 cases, as reported by health authority officials, more than one individual was involved in the decision-making process and in $38 \%$ of these (9/24), the BWA was issued after multiple deliberations between those involved. In the subset of 14 water systems, where both water suppliers and health authority officials were interviewed, $50 \%$ and $71 \%$ mentioned that multiple individuals were involved with the decision, respectively. Of these, 
$57 \%$ (4/7) of water suppliers and 50\% (5/10) of health authority officials indicated that multiple deliberations took place before issuing the subset of 14 BWAs. There was no notable relationship observed between the involvement of more than one person in decision making and the size of the water system.

Table 9: Individuals who were involved in the decision to issue boil water advisories

\begin{tabular}{|c|c|c|}
\hline $\begin{array}{l}\text { Primary Decision-Makers } \\
\text { (\% water systems) }^{*}\end{array}$ & Consultant Roles & $\begin{array}{l}\text { Stakeholders/System-leve } \\
\text { Information Providers }\end{array}$ \\
\hline $\begin{array}{ll}\text { - } & \text { Environmental Health } \\
\text { Officers }(45 \%) \\
\text { - } \\
\text { Drinking Water Officers }{ }^{1} \\
(16 \%) \\
\text { - } \text { Drinking Water Leaders } \\
(13 \%) \\
\text { - Public Health Inspectors }{ }^{2} \\
(6 \%) \\
\text { - Drinking Water Quality } \\
\text { Specialists }(6 \%) \\
\text { - Water Suppliers }(6 \%)\end{array}$ & $\begin{array}{l}\text { - Public Health or Drinking } \\
\text { Water Engineers (from } \\
\text { health authority) } \\
\text { - Engineers (from water } \\
\text { system) } \\
\text { - Drinking Water Leaders } \\
\text { - Chief Environmental } \\
\text { Health Officers } \\
\text { (Supervisors) } \\
\text { - Medical Health Officers }\end{array}$ & $\begin{array}{l}\text { - Water suppliers (or water } \\
\text { system representatives) }\end{array}$ \\
\hline
\end{tabular}

* Out of 31. At 2 water systems (6\%) no primary decision-maker was identified

1: All Drinking Water Officers interviewed were also Environmental Health Officers, but this may not be the case across the province.

2: The title 'Public Health Inspector' is equivalent to Environmental Health Officer, but the table reflects information as reported by participants in interviews

In 9 of the 31 BWAs (29\%), the health authority official reported that the water supplier was involved in the decision-making process. In the subset of 14 BWAs, 5 (36\%) and $6(43 \%)$ health authority officials and water suppliers, respectively, indicated that the water supplier or someone from the water system was involved. In 2 cases, the health authority official stated that the water supplier was involved when the water supplier stated otherwise.

Among water systems where multiple individuals were involved in the decision-making process, no health authority official or water supplier answered in the affirmative, when asked if any of the members involved in the decision, the interviewee included, were openly opposed to issuing the BWA $(0 / 24$ and $0 / 7$, respectively).

Eighty-seven percent of all health authority officials interviewed reported that there were no reasons not to issue the BWA. The officials who did consider some reasons not to issue the BWA, largely reflected the lack of definitive evidence or supporting information on hand and the 
need to act for precautionary purposes. For instance at one water system, there was no inspector on site who knew the system well; in another case, the health authority official was dealing with contradictory and inconclusive water bacteriological results. One other official considered not issuing the BWA as the water system was new and the positive bacteriological results (which were the reason for considering a BWA in the first place) were sporadic. One water supplier mentioned that his reason not to issue was based on his knowledge of the public's opinion and perception of their water supply. In his words: "[The] people didn't want it. The people wanted the choice of their water and no one ever had any sickness from it". This community was receiving raw drinking water from a mountain spring water source and were opposed to having any form of treatment installed at the system (which would be a requirement for the BWA's removal).

When asked if any alternative actions were considered in place of issuing a BWA, such as issuing some other form of public notification, the majority of the officials (27/31) responded "no" while also mentioning that some accompanying actions were considered (such as trucking water in or using alternative forms of disinfection), but these were in addition to issuing the BWA and did not replace the need for a BWA. Three officials and two water suppliers mentioned that alternative actions were considered, such as issuing a water quality advisory in place of a BWA. In one community, where the water was not being disinfected, the option of initiating the maintenance of a disinfection residual was discussed, as an alternative to issuing a BWA, but this idea was later decided against due to pressures from community members.

\subsubsection{The use of guidelines}

The majority of health authority officials reported that they do have access to guidelines to support their decision on when to issue or rescind BWAs (Figure 6a and b, respectively). Water supplier's access to and/or usage of guidelines was not as prominent. 


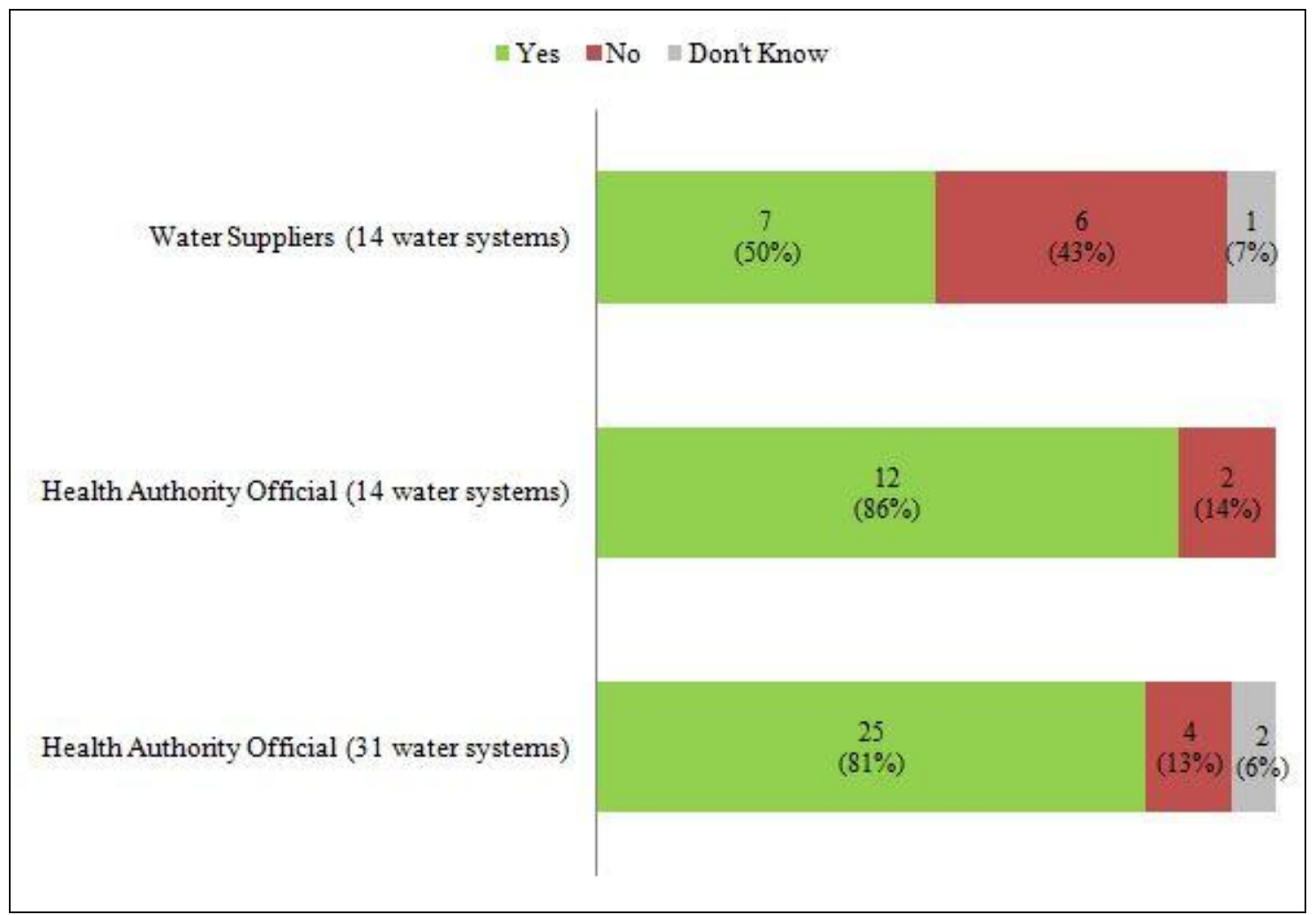

Figure 6a: Do you follow any written guidelines on when to issue boil water advisories?

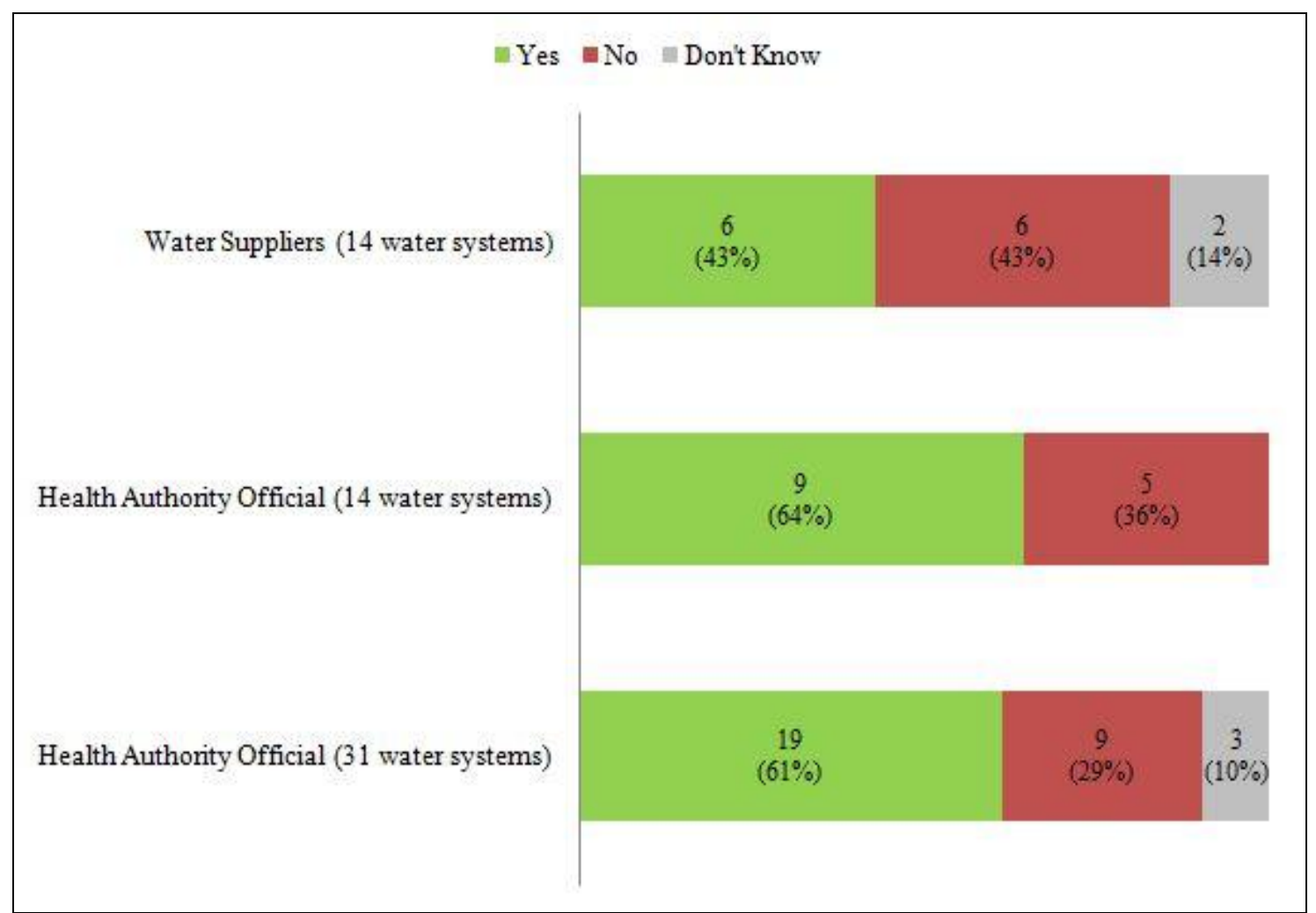

Figure 6b: Do you follow any written guidelines on when to rescind boil water advisories? 
The following documents were referenced as resources used by health authority officials to guide the issuing and rescinding of BWAs (note: the health authority level documents were only referenced by those participants in that specific health authority):

At the Local/Health Authority Level:

- Fraser Health Authority's Guideline: Issuing and Rescinding a Drinking Water Public Notice

- Interior Health Issue Paper: Drinking Water Public Notification Policy and Guidance Recommendation

- Northern Health Authority: Guideline for Boil Water Notice Decision Process

- Northern Health Authority, Drinking Water Program Section, Public Health Protection Policy Manual: Orders to Provide Public Notice About Drinking Water (PHP410) and Notification Protocol (PHP420)

- Northern Health Authority: Policy and Procedures Handbook - Drinking Water Protection Act (Appendix 21)

At the Provincial/Territorial Level:

- The Drinking Water Protection Act and Regulation

- The Drinking Water Officer's Guide

- Ministry of Health Services: Decision Tree for Responding to a Turbidity Event in Unfiltered Drinking Water

At the Federal Level:

- Health Canada: Guidance for Issuing and Rescinding Boil Water Advisories

Table 10 provides an overview and comparison of the information contained in the above listed resources, with respect to the issuing and removal of BWAs. It is important to note that the above list is not an exhaustive one. It includes only those documents cited in interviews with health authority officials as reference tools to which they have access.

Water suppliers who indicated that they used guidelines to issue and/or rescind BWAs referred to water system-specific emergency response plans or supplemental reference material provided by their health authority, for example the IHA's Turbidity Education and Notification Campaign. 
Table 10: A comparison of the content of regulations, policies and other supporting documents, as referenced by health authority officials, for use in guiding the management of public notifications, specifically the issuing and rescinding of BWAs

\begin{tabular}{|c|c|c|c|c|c|c|c|c|}
\hline & $\begin{array}{l}\text { Defines the } 3 \\
\text { types of } \\
\text { public } \\
\text { notifications } \\
\text { and when } \\
\text { each may be } \\
\text { issued }\end{array}$ & $\begin{array}{l}\text { Mentions } \\
\text { responsibilities } \\
\text { of health } \\
\text { authority and } \\
\text { water system } \\
\text { with respect to } \\
\text { BWAs }\end{array}$ & $\begin{array}{l}\text { Provides } \\
\text { steps to take } \\
\text { when } \\
\text { deciding to } \\
\text { issue BWA }\end{array}$ & $\begin{array}{l}\text { Provides } \\
\text { criteria or } \\
\text { factors to } \\
\text { consider } \\
\text { into } \\
\text { decision to } \\
\text { issue BWA } \\
\end{array}$ & $\begin{array}{l}\text { Provides } \\
\text { information } \\
\text { on actions } \\
\text { to take after } \\
\text { issuing } \\
\text { BWA }\end{array}$ & $\begin{array}{l}\text { Provides } \\
\text { steps to take } \\
\text { when } \\
\text { deciding to } \\
\text { lift BWA }\end{array}$ & $\begin{array}{l}\text { Provides } \\
\text { criteria or } \\
\text { factors to } \\
\text { consider } \\
\text { into } \\
\text { decision to } \\
\text { lift BWA } \\
\end{array}$ & $\begin{array}{l}\text { Provides } \\
\text { templates of } \\
\text { BWA } \\
\text { notices or } \\
\text { orders }\end{array}$ \\
\hline \multicolumn{9}{|l|}{ Federal Level } \\
\hline $\begin{array}{l}\text { Health Canada: Guidance for Issuing and } \\
\text { Rescinding Boil Water Advisories }\end{array}$ & $x_{3}$ & $x$ & $\checkmark_{6}$ & $\checkmark$ & $\checkmark_{6}$ & $\checkmark_{6}$ & $\checkmark$ & $x$ \\
\hline \multicolumn{9}{|l|}{ Provincial/Territorial Level } \\
\hline $\begin{array}{l}\text { The Drinking Water Protection Act and } \\
\text { Regulation }\end{array}$ & $x$ & $\checkmark_{1}$ & $x$ & $x$ & $x$ & $x$ & $x$ & $x$ \\
\hline The Drinking Water Officer's Guide & $\checkmark$ & $\checkmark$ & $x$ & $x_{2}$ & $x$ & $x$ & $\checkmark_{1}$ & $\checkmark$ \\
\hline $\begin{array}{l}\text { Ministry of Health Services: Decision } \\
\text { Tree for Responding to a Turbidity Event } \\
\text { in Unfiltered Drinking Water }\end{array}$ & $\checkmark$ & $x$ & $\sqrt{6,7}_{6}$ & $x$ & $\sqrt{6,7}_{6}$ & $\sqrt{6,7}_{6}$ & $x$ & $x$ \\
\hline \multicolumn{9}{|l|}{ Local/Health Authority Level } \\
\hline $\begin{array}{l}\text { Fraser Health Authority's Guideline: Issuing } \\
\text { and Rescinding a Drinking Water Public } \\
\text { Notice }\end{array}$ & $\checkmark$ & $\checkmark$ & $\checkmark_{1}$ & $\checkmark_{1}$ & $\checkmark_{1}$ & $\checkmark_{1}$ & $\checkmark_{1}$ & $\checkmark$ \\
\hline $\begin{array}{l}\text { Interior Health Issue Paper: Drinking Water } \\
\text { Public Notification Policy and Guidance } \\
\text { Recommendation }\end{array}$ & $\checkmark$ & $\checkmark$ & $x$ & $x_{2}$ & $\checkmark$ & $x$ & $\checkmark_{1}$ & $x$ \\
\hline
\end{tabular}




\begin{tabular}{|c|c|c|c|c|c|c|c|c|}
\hline & $\begin{array}{l}\text { Defines the } 3 \\
\text { types of } \\
\text { public } \\
\text { notifications } \\
\text { and when } \\
\text { each may be } \\
\text { issued }\end{array}$ & $\begin{array}{l}\text { Mentions } \\
\text { responsibilities } \\
\text { of health } \\
\text { authority and } \\
\text { water system } \\
\text { with respect to } \\
\text { BWAs }\end{array}$ & $\begin{array}{l}\text { Provides } \\
\text { steps to take } \\
\text { when } \\
\text { deciding to } \\
\text { issue BWA }\end{array}$ & $\begin{array}{l}\text { Provides } \\
\text { criteria or } \\
\text { factors to } \\
\text { consider } \\
\text { into } \\
\text { decision to } \\
\text { issue BWA }\end{array}$ & $\begin{array}{l}\text { Provides } \\
\text { information } \\
\text { on actions } \\
\text { to take after } \\
\text { issuing } \\
\text { BWA }\end{array}$ & $\begin{array}{l}\text { Provides } \\
\text { steps to take } \\
\text { when } \\
\text { deciding to } \\
\text { lift BWA }\end{array}$ & $\begin{array}{l}\text { Provides } \\
\text { criteria or } \\
\text { factors to } \\
\text { consider } \\
\text { into } \\
\text { decision to } \\
\text { lift BWA } \\
\end{array}$ & $\begin{array}{l}\text { Provides } \\
\text { templates of } \\
\text { BWA } \\
\text { notices or } \\
\text { orders }\end{array}$ \\
\hline $\begin{array}{l}\text { Northern Health Authority: Guideline for Boil } \\
\text { Water Notice Decision Process }\end{array}$ & $x_{3}$ & $x$ & $x$ & $\checkmark$ & $\checkmark_{4}$ & $x$ & $x$ & $x$ \\
\hline $\begin{array}{l}\text { Northern Health Authority Public Health } \\
\text { Protection Policy Manual: Orders to Provide } \\
\text { Public Notice About Drinking Water (PHP410) } \\
\text { and Notification Protocol (PHP420) }\end{array}$ & $x$ & $\checkmark_{1}$ & $x$ & $x$ & $x_{5}$ & $x$ & $x$ & $\checkmark$ \\
\hline $\begin{array}{l}\text { Northern Health Authority: Policy and } \\
\text { Procedures Handbook - Drinking Water } \\
\text { Protection Act (Appendix 21) }\end{array}$ & $\checkmark$ & $x$ & $x$ & $x$ & $x$ & $x$ & $x$ & $\checkmark$ \\
\hline
\end{tabular}

1: Information is general to any type of public notice and not specific to BWAs

2: Includes a list of factors to consider when deciding what type of public notification to issue; not for when deciding to issue a BWA or not

3: Only information pertaining to BWAs was included

4: Only specific to BWAs due to poor bacteriological quality of drinking water

5: Only information with respect to who to report to or consult with after issuing a BWA was provided

6: In the form of a decision-tree

7: Information was specific to public notifications, including BWAs, due to turbidity 


\subsubsection{Public communication}

When health authority officials were asked who was responsible for communicating the BWA at each of the 31 water systems to the public, $30(97 \%)$ of them stated that the water supplier is solely responsible. No official said that the health authority is solely responsible, but one official mentioned that the responsibility lies in the hands of both the health authority and water supplier. In the subset of 14 water systems, 13 of the health authority officials said the onus is on the water suppliers. Only $11(79 \%)$ of all water suppliers interviewed, on the other hand, recognized that it is their responsibility to communicate the BWA to the system's consumers. Three water suppliers $(21 \%)$ stated that the responsibility lies with the health authority, but no water supplier reported the responsibility as shared between both the health authority and water supplier. The difference between the health authority officials' response and the water supplier's response was not statistically significant. Figure 7 illustrates the availability and usage of documentation to assist in the development of the BWA message.

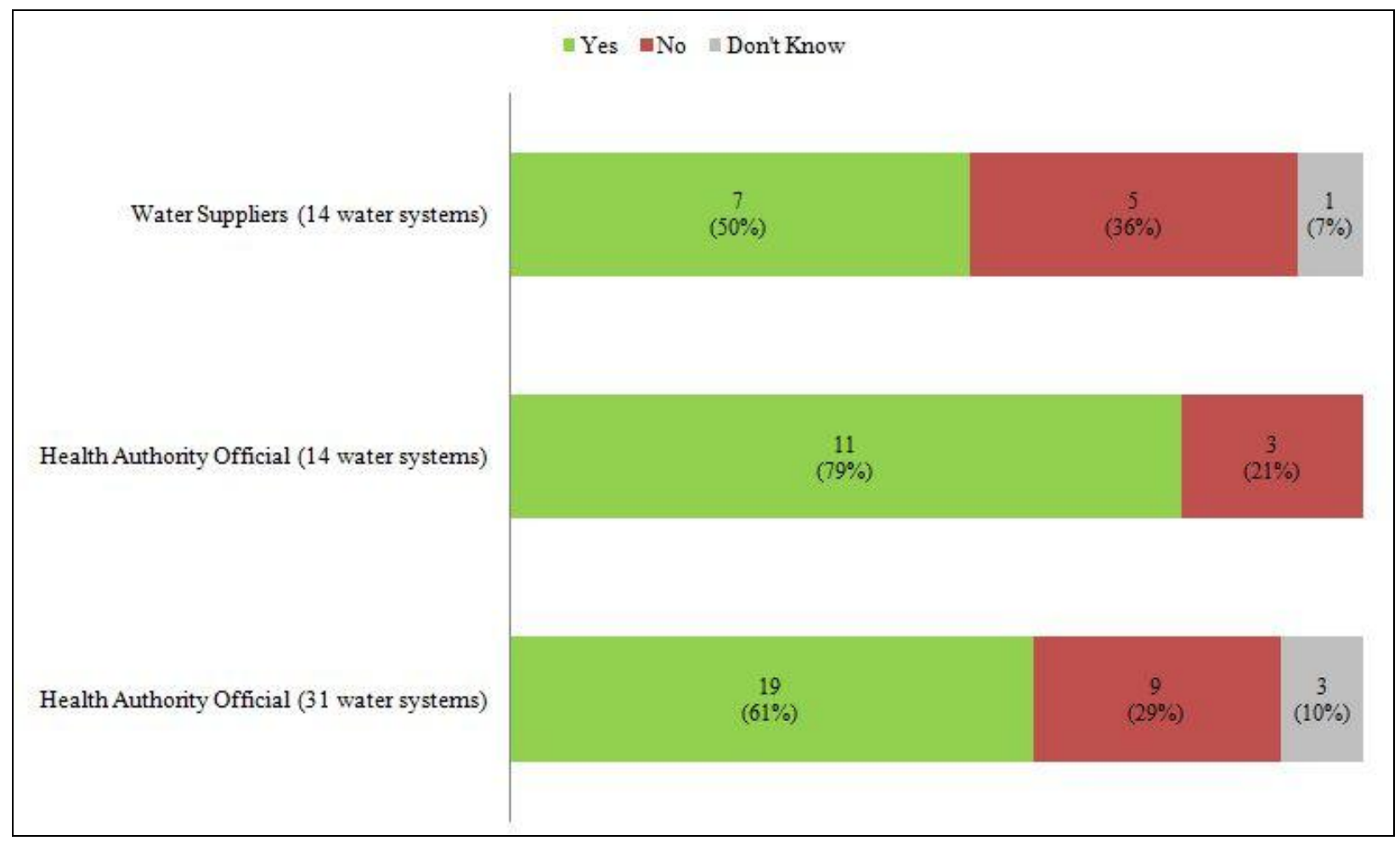

Figure 7: Is there a template or checklist available that lists what information to include in a boil water advisory? 


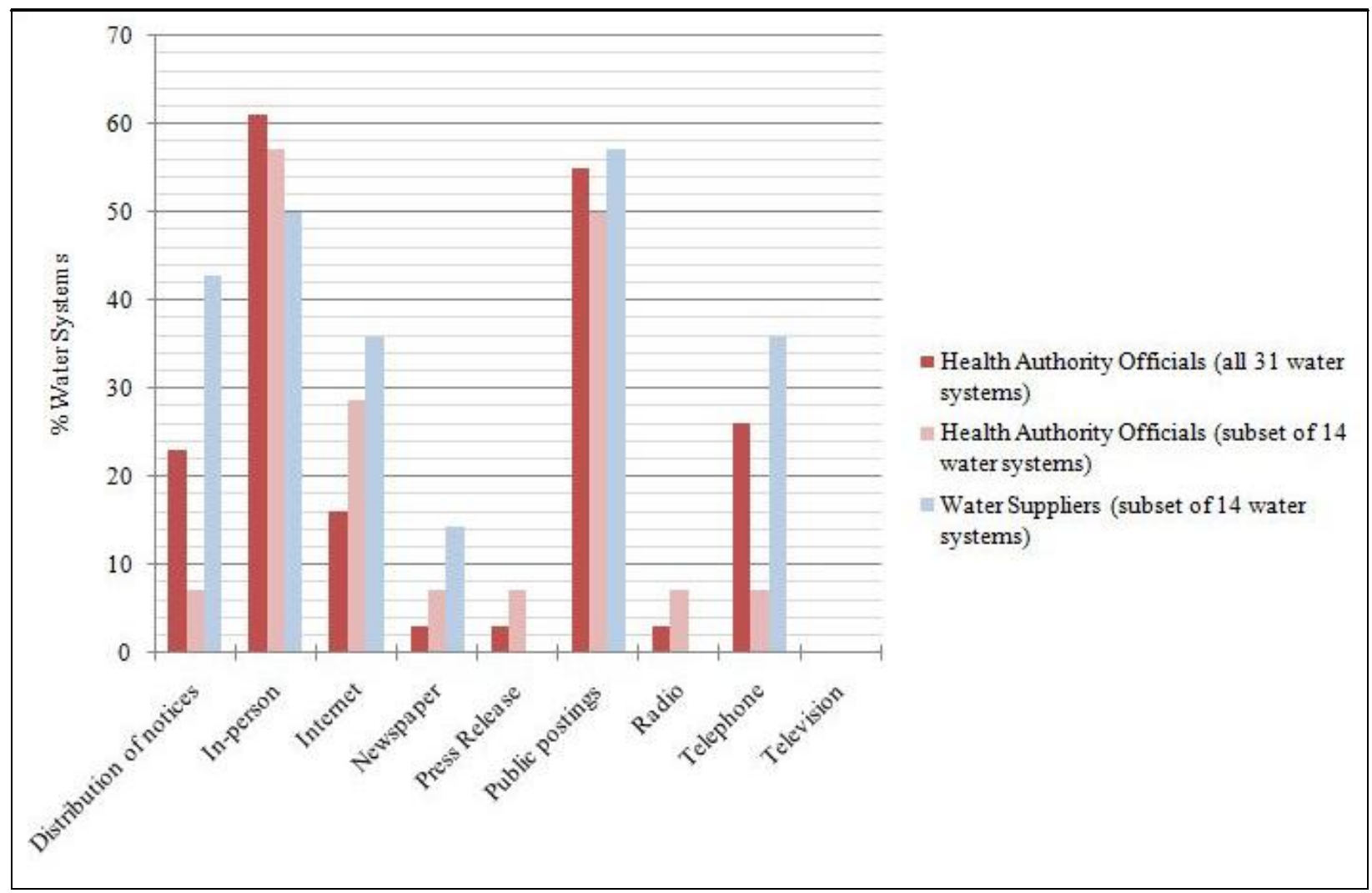

Figure 8: The means by which the boil water advisory was communicated to the public as reported by health authority officials and water suppliers

* Distribution of notices included any written documentation, such as letters or flyers; Internet included notifications via website and/or email. In-person included door-to-door canvassing and annoucements in group meetings with public (example: in annual general meetings or at camp orientation)

Figure 8 illustrates the methods by which the public was informed of the BWA and Table 11 details the specific information and recommendations that the BWA message included. The total number of different methods used to communicate the BWA (at 31 water systems) to the public did not differ according to long-term or short-term classification; an average of 2.1 methods were used in short-term BWAs and 1.8 in long-term. In $71 \%$ of all 31 water systems, at least two different methods to communicate the BWA were implemented, according to health authority officials. According to water suppliers at the 14 water systems, $86 \%$ had used as few as 2 and at most 6 different methods to communicate the BWA. While there was no major overlap between any two methods to communicate the BWA (at all 31 water systems), informing the public in-person and via public postings were done together $30 \%$ of the time. 
Only $29 \%$ of all health authority officials and $79 \%$ (11/14) of all water suppliers interviewed had a record of the BWA as it was communicated to the public. It is therefore important to keep in mind that the details on the BWA message provided were dependent on the participant's memory and/or assumptions of what may have been disseminated to the public, especially by those lacking records.

Boiling water is more commonly recommended in BWAs than bottled water use. All health authority officials reported that the BWA message included information on what to do to avoid health risk and for all cases it was recommended that the public boil their water. 5 (38\%) water suppliers recommended boiling over bottled water use, $4(31 \%)$ recommended both and 3 (23\%) recommended bottled water over boiling water in the BWA message to the public. Some reasons for why bottled water use was recommended rather than boiling water for drinking included: aesthetic reasons (tap water didn't taste good due to high iron content and boiling wouldn't help), the water supplier was providing bottled water anyway (as in camps and resorts), and the fact that some water suppliers would rather encourage their consumers to depend on an alternative source of drinking water (like bottled water) or install their own water treatment (such as point-of-use/point-of-entry treatment systems) in their home as a better and more permanent solution, than having to boil tap water. On average, health authority officials reported more information in the BWA message if the BWA was long-term (the mean number of types of information given in long-term BWAs was 3.3 and 2.5 in short-term) but this difference was not statistically significant $(0.85$ mean difference, $\mathrm{t}$-value $=1.17, \mathrm{df}=27, \mathrm{p}=0.25)$. Recommending the public to use some other form of water treatment as a measure to protect health was also only mentioned in the long-term BWAs and not for any short-term ones.

When asked if there were any complaints or questions about the clarity of the BWA message, $24(77 \%)$ of all health authority officials said "no". Only 1 official came across questions asking for clarification on what the BWA was and what some of its recommendations meant. Thirteen out of fourteen water suppliers, who were asked the same question, indicated that they did not come across any complaints on the clarity of the message. Timeliness of the receipt of the BWA message did not seem to be an issue as most health authority officials (84\% of 31) and water suppliers (93\% of 14) did not encounter any public complaints on this. 
According to health authority officials, the number of times the BWA message was communicated to the public (ie. reminders) was low - in $65 \%$ of the systems, the BWA was communicated to the public just once, at time of issue or at time of first contact with a member of public (such as upon camp or resort registration), but this was accompanied by public postings remaining in place. In $10 \%$ of the systems, the BWA message was reiterated at least annually in gatherings such as annual general meetings. Among the water suppliers interviewed, 7 (50\%) mentioned that the BWA was communicated once, 3 (21\%) stated that the message was reiterated daily, and the remaining said that annual reminders were given. 
Table 11: Information provided to public about the boil water advisory at a subset of water systems as reported by health authority officials and water suppliers

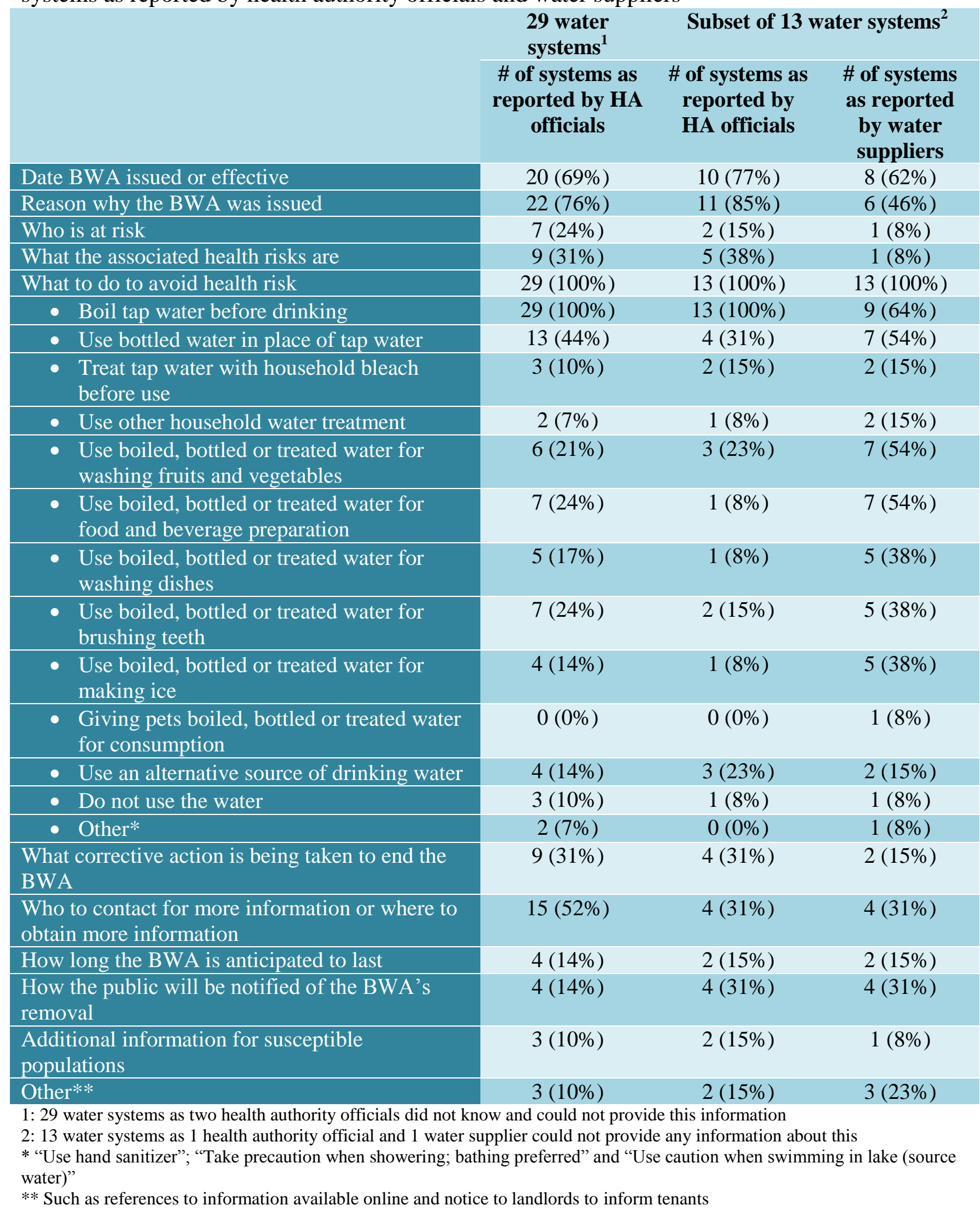




\subsubsection{Public compliance}

Figure 9 compares the perception of public compliance among health authority officials and water suppliers. Interestingly, it appears that health authority officials have less faith in the public, in terms of their compliance towards BWAs, than water suppliers do, as a greater proportion of water suppliers believed that the public is complying with the BWA than health authority officials. This difference, however, was not statistically significant (Chi-square $=2.74$, $\mathrm{df}=2, \mathrm{p}$-value $=0.25)$.

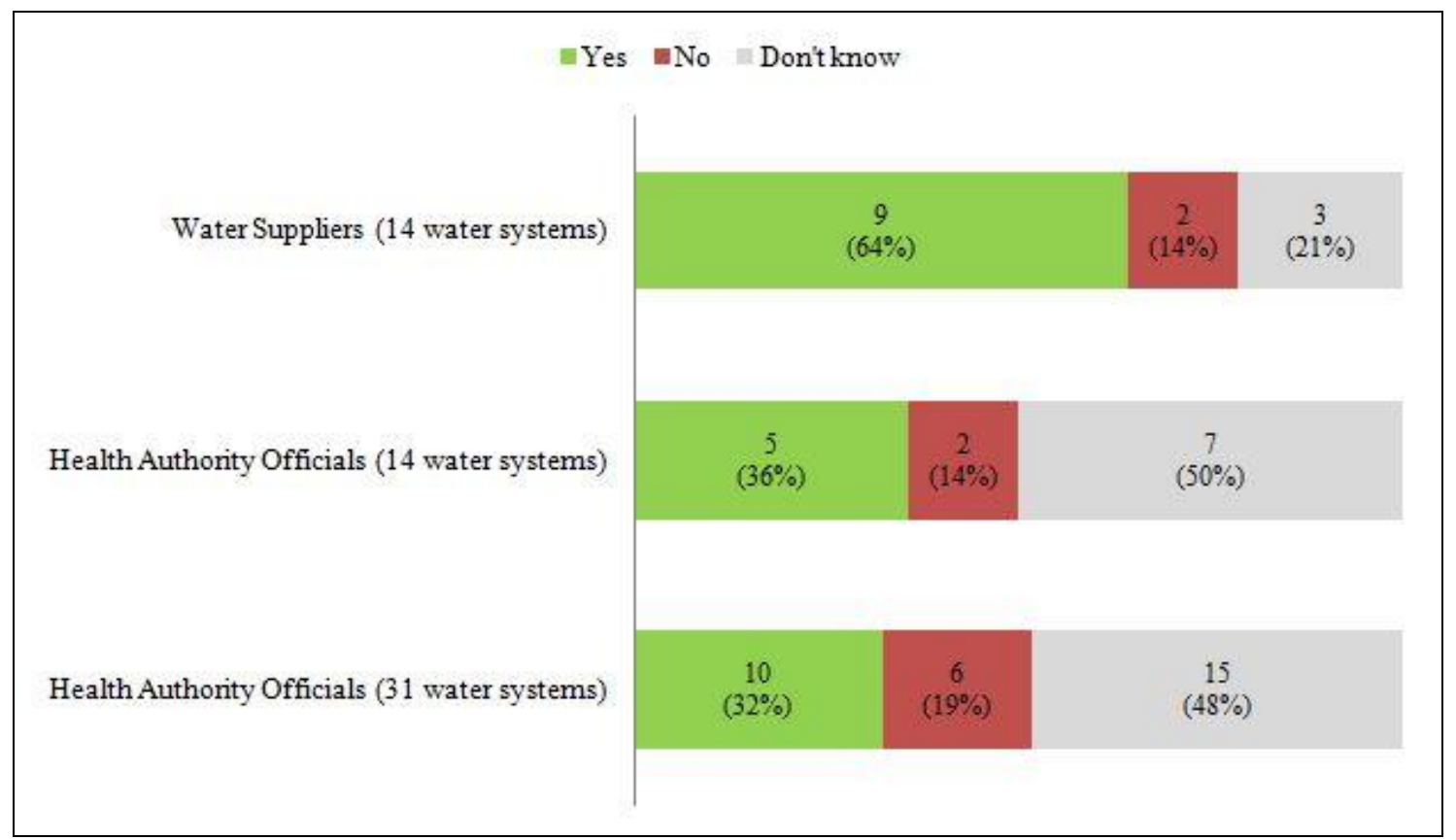

Figure 9: Do you think the public complied with the boil water advisory?

In Figure 10, data on whether or not efforts were taken by health authority officials and water suppliers to ensure public compliance is shown. Health authority officials admitted that not much could be done on their end to ensure public compliance, as stated by one official:

No,[ensuring public compliance is] very difficult. You'd have to have staff go out and visit each family to ask what they're doing about [the boil water advisory]. And that's a real weakness. We don't know how many people heed the warning and how many don't. It's a weakness in our data.

The two health authority officials who took extra measures (in the form of increased follow up with water supplier and frequent onsite visits) to address compliance did so because one system served a resort and the other a school; the officials wanted to ensure those who can't 
make an informed choice (children) or those not a part of the local community (tourists) were being taken care of.

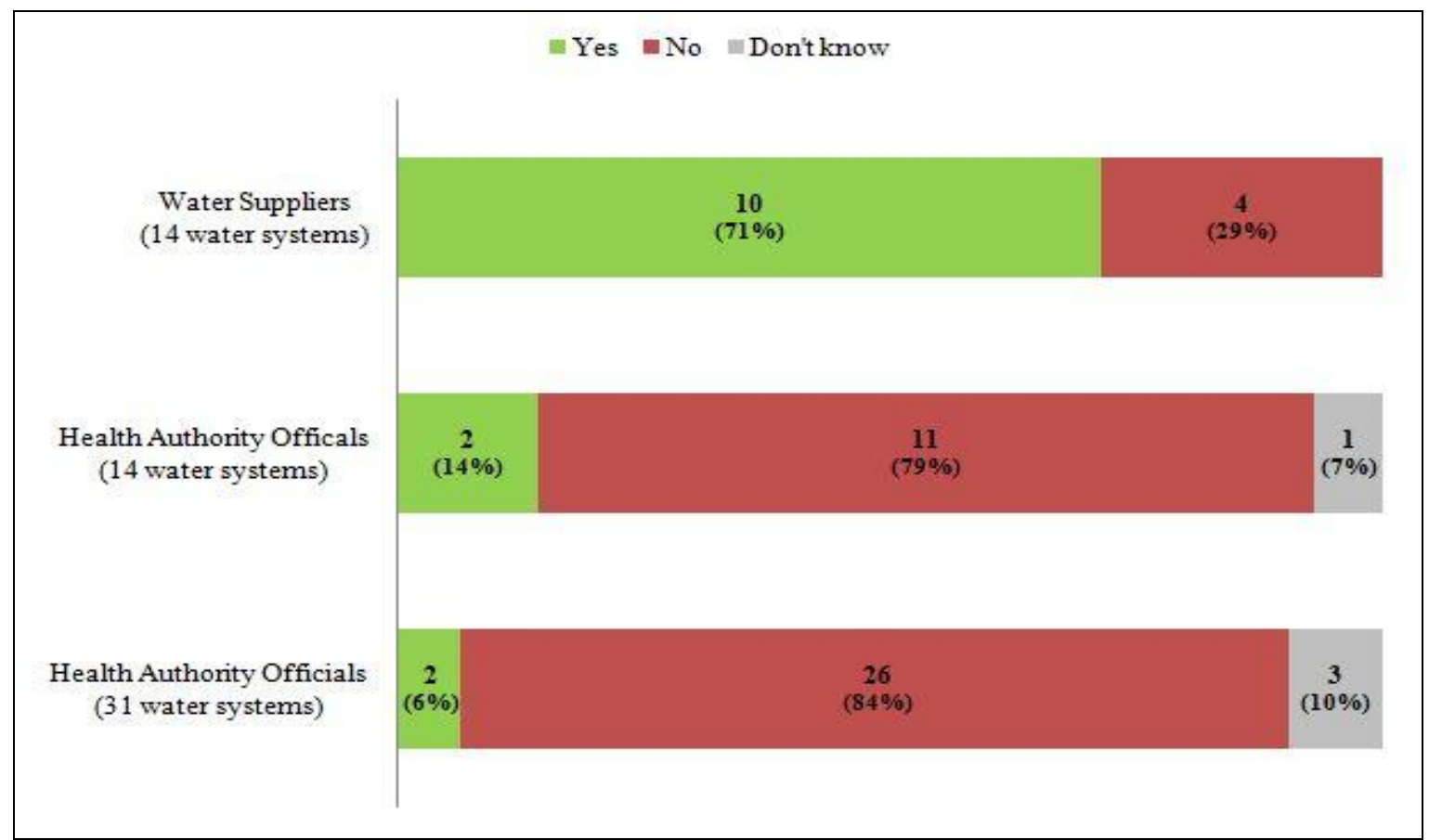

Figure 10: Were any efforts taken to ensure public compliance?

The greater responsibility for ensuring public compliance was on the shoulders of water suppliers, who were deemed in charge of the public communication of BWAs to those being served water by their system. To encourage public compliance, water suppliers relied on the use of signage and frequent verbal reminders. At water systems primarily serving businesses (such as resorts or children's summer camps), additional measures included:

- Ensuring proper signage at each public tap or faucet

- Kitchen staff being reminded about BWA

- Kids at summer camp being supervised when using washroom (so they don't drink faucet water)

- Ensuring visitors are informed about the BWA upon arrival or registration

- Supplying free bottled water to visitors 


\subsubsection{The consequences}

Ninety percent of all health authority officials and $86 \%$ of all water suppliers interviewed reported that there are criteria or benchmarks set in place to help guide and indicate when the BWA can be lifted. However, it was not known whether these criteria were established at the time the BWA was issued. Figure 11 diagrammatically illustrates the steps needed to lead to the removal of BWAs. In Table 4 above, information about what main actions had been taken since the issuing of the BWA and what was pending, was tabulated for each water system.

About half of all health authority officials interviewed (52\%) stated that the primary responsibility for ensuring that the BWA was moving towards removal and any problems that triggered the issuing of the BWA, were being addressed, was in their hands and $42 \%$ put the onus on the water supplier. One health authority official stated that both are responsible and one other replied that s/he did not know. In contrast, $79 \%$ of water suppliers said they themselves are responsible while $14 \%$ put the onus on health authority officials; one water supplier said the onus is on both individuals. The Kappa statistic for agreement beyond chance was 0.21 , indicating poor agreement between health authority officials and water suppliers on who is ultimately responsible (crude percent agreement was 36\%). In $19 \%$ of the water systems, a ballpark date by which the BWA's removal was anticipated was set, as reported by health authority officials. In the subset of 14 water systems, $14 \%$ of health authority officials and $43 \%$ of water suppliers indicated that there was a date set. There was agreement that health authority officials are ultimately the ones who decide on when to rescind the BWA; 93\% of all participating health authority officials and $86 \%$ of all water suppliers indicated this.

All health authority officials and water suppliers mentioned that intercommunication and following-up did take place (at least once) after the issuing of the BWA. In most cases, water suppliers were reminded of corrective actions required by health authority officials and any progress made since was reviewed. In a of couple instances, the health authority officials reported that there had been no communication with the water supplier since their last attempt to contact them, which was a few weeks before the time of interview. 
In about half of the cases, health authority officials (45\%) and water suppliers (50\%) reported that members of the public were being regularly updated on the current situation with the BWA. These updates were largely conveyed when there was something to update the public about, and usually upon personal contact or through updates online via health authority and/or water system websites. There was not much contact made to health authority officials and water suppliers by the public for matters regarding the BWA. Only 13\% (4/31) of health authority officials and $21 \%(3 / 14)$ water suppliers reported that they were contacted by the public.

\subsubsection{A snapshot of the current situation of boil water advisories in BC}

Figure 11 attempts to illustrate the overarching goal of this study - to provide some insight on the situation with BWAs in BC - from why they are issued, what actions are required to lead to its removal and what challenges are in the way that hinder their timely removal. 


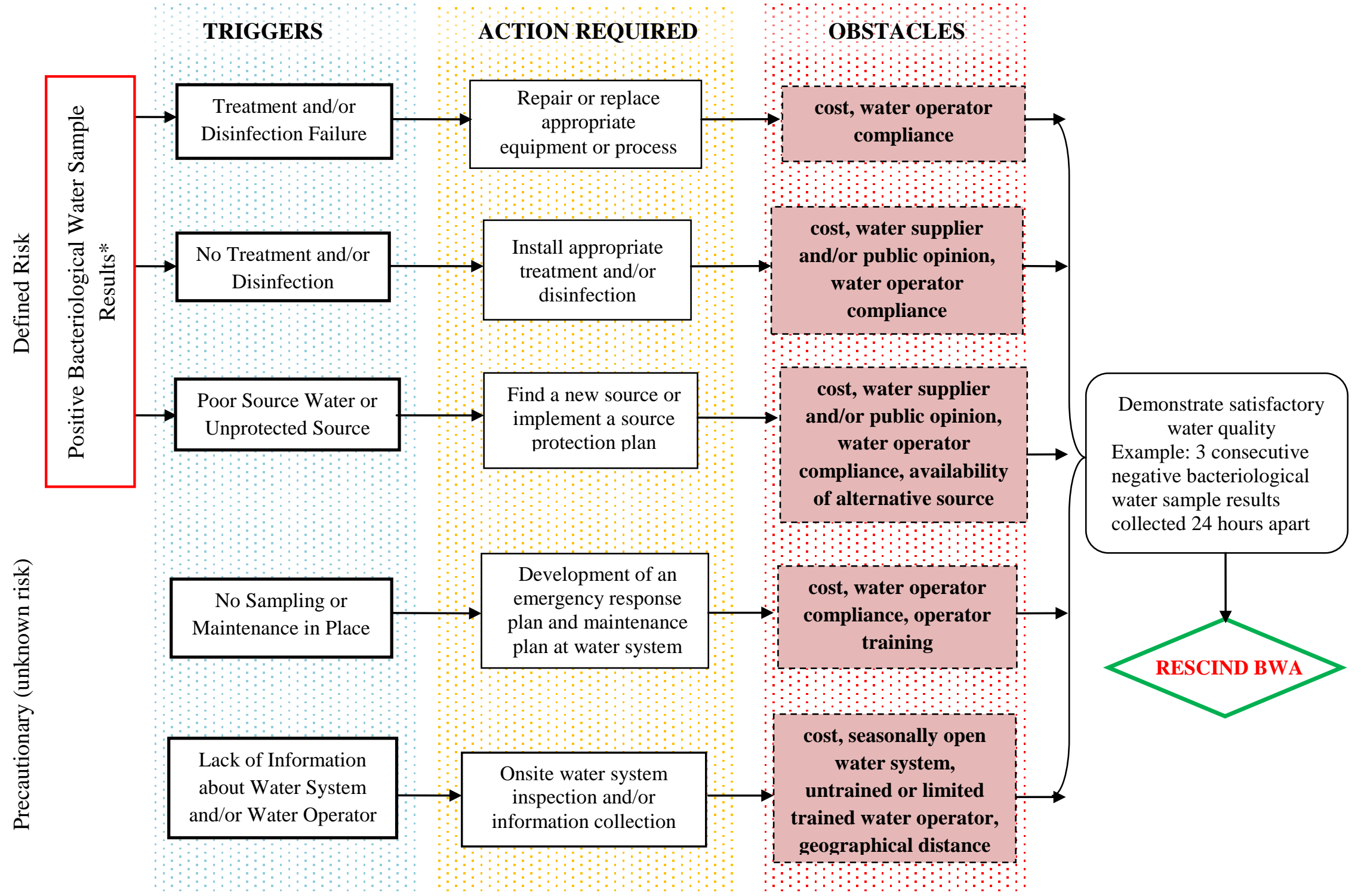

Figure 11: Common triggers leading to the issuing of BWAs, the specific actions required and the obstacles that hinder removal, as identified in this study. (* Lab tests showing evidence for E.coli, fecal coliform and/or total coliform) 


\section{Discussion of Results}

This study is the first of its kind to investigate the process by which BWAs are issued in $\mathrm{BC}$, including the factors and criteria considered by decision-makers, how BWAs are communicated to the public and what actions are taken after, or are pending, since the advisory's issuance, to allow for its removal. The unique methodology involving the collection of data from both health authority officials and water suppliers allowed for an interesting comparison of perceptions and a more complete picture of the story of BWAs at water systems in four of BC's health authorities. The nature of the study also allowed for the collection of detail rich narratives that described some of the main challenges and obstacles faced by health authority officials and water suppliers in managing BWAs.

\subsection{Reasons to Issue Boil Water Advisories \& The Decision-Making Process}

The main reasons for issuing a BWA, as determined in this investigation, were: 1) evidence of water contamination with bacteria (E.coli, fecal or total coliforms) via lab test results $(81 \%) ; 2$ ) the lack of water treatment or disinfection in place $(29 \%) ; 3$ ) source waters being of poor quality or being unprotected $(6 \%) ; 4)$ the lack of information available to health authority officials about the water system under question $(6 \%) ; 5)$ the failure to treat or disinfect water due to mechanical breakdown of equipment (3\%); and 6) the lack of any sampling and/or maintenance plan in place at the water system (3\%). Other than the unavailability of information about the system, the aforementioned reasons coincided with those cited as known reasons for issuing BWAs in government reports and guidelines $(8,23)$.

Water suppliers seem more inclined to indicate water quality issues alone or in addition to evidence for risk to health, as reasons for issuing a BWA. This may be reflective of water suppliers' awareness for the need to maintain aesthetic quality of water, especially since consumers tend to measure the safety of their drinking water based on taste, odours or discolouration (47). In a paper by Hrudey and Hrudey (2007), it was emphasised that water suppliers should not only aim to meet the minimums set in drinking water regulations, but also equally aim meet the consumer's highest standards for taste, odour and appearance (47). Aesthetic factors could be used as early warning signs for an impending water safety issue, as 
evidence indicates that the impact of some past outbreaks may have been reduced had such warning signs been pursued early on by water suppliers (47).

Water quality advisories? I personally struggle with this term... I can't tell you where I would use this over [a] boil water advisory. [In my] person opinion, I always felt concerned for my communities because it's difficult for them to decide what to do - when to use this public notification over others.

- An Environmental Health Officer

To know when to issue a BWA, or any type of public notification, first depends on the decision-makers' awareness of what types of public notices they have at their disposal and their knowledge and understanding of what each public notification is, by definition and in practice. The Drinking Water Officer's Guide theoretically describes the three types of public notices that are issuable on water systems in BC (8). Despite this, not all health authority officials and water suppliers were aware of these notices and correctly defining them. Perhaps this speaks of lack of experience in facing situations where the other types of public notifications might be used.

"Water quality advisories" and "do not drink advisories" are not issued as often as BWAs in all health authorities. There is a need for consistent terminology and definitions, across BC as well as a need for better awareness among decision-makers on the different types of public notifications. This becomes especially important as such confusion may affect the public's interpretation and perception of risk as well as their likelihood to comply, as was hypothetically demonstrated in one study (40).

Let's be very clear about what triggers a 'boil water advisory', a 'water quality advisory', or a 'stop water use' before those are instituted. Another health region had a positive count out of a garden hose. Why was the river [being sampled] out of a garden hose?... Anyway, they got an E.coli so they put a boil water advisory in place. Was it warranted to issue a boil water advisory with just one result? My first question would be how did you sample? The [water supplier] could have contaminated the sample. What is the history of that sampling location? You got to answer all those questions before you formulate a conclusion and issue an advisory... When I hear things like that I just shake my head. No wonder the public loses faith on what we're trying to say... we lose credibility because we're not all doing the same thing. Some health authorities are doing some things differently. That to me is not proper notification to the public. Questions have to be answered... A checklist, if you have it, or a guideline or a policy - they have to go through that and when they do, it's more of a sound decision... There needs to be consistency among health authorities to limit confusion.

An Environmental Health Officer

The decision-making processes varied from one case to the next - within and across health authorities. A different set of criteria or factors were considered and different members were involved in the decision-making process, depending on the water system in consideration. 
The four most common factors considered by health authority officials, when deciding to issue a BWA, were: 1) the absence of water treatment or disinfection - either because the system did not have any in place or due to its temporary malfunctioning $(90 \%) ; 2)$ evidence for poor microbiological quality $(64 \%)$; 3 ) the water source $(58 \%)$; and 4) the history of the water system (48\%). Public inconvenience was only considered by water suppliers. Although data from the four health authorities were not compared with each other with respect to the decision-making process for issuing BWAs, some health authority officials did mention that the processes are not consistent and standardized (as in the above boxed comment).

Documentation to help support the issuing and rescinding of BWAs is available, but not all health authority officials and water suppliers are aware of such documents and not all utilise them. Health Canada's Guidance for Issuing and Rescinding Boil Water Advisories, for instance, lists conditions that may trigger the need for a BWA and conditions for when BWAs are usually rescinded (23). According to this guidance document, BWAs should be issued immediately upon evidence for the confirmed presence of E.coli in water, as this microorganism is an indicator of human or animal fecal contamination and the possible presence of other pathogenic microorganisms (23). Health authority officials and water suppliers may issue a BWA upon evidence indicating the presence of fecal coliform in water, but since certain species in this group are ubiquitous in nature and not indictors of fecal contamination, it is recommended that the identity of the coliform be determined prior to considering the need for a BWA (23). Species in this group, other than E.coli, may indicate bacterial re-growth in the distribution system (23). The presence of total coliform does not indicate a health risk, but BWAs may be issued if remedial measures, such as flushing the system or increasing chlorine residuals do not prove effective (23). In relation to this, the DWPR in Schedule A prescribes standards for potable water with respect to E.coli, fecal coliform and total coliform in water samples (48). In $\mathrm{BC}$, the standards indicate that no fecal coliform and E.coli be detectable in water samples and at least $90 \%$ of the samples in a 30 day period not have any detectable total coliform (48). Interestingly, whereas most published research on BWAs has focused on Cryptosporidium as indicators for the need for BWAs, testing for these water parasites is not prescribed in BC, and is not factored into the decision-making process, despite $\mathrm{BC}$ having a high prevalence of these parasites $(33,37)$. Current methodology for parasite testing is only indicative of the presence or 
absence of the parasite and doesn't indicate its viability, so the use of such data as triggers for issuing BWAs may not be appropriate.

With respect to bacterial testing, the guidelines and the regulations do provide fairly clear directives on when BWAs can be issued. However, there is not much instruction on situations where there is uncertainty about the presence of risk. For instance, does the presence of one positive E.coli sample establish enough risk to justify the need for a BWA? The possibility of false-positive water samples, which has been mentioned by many health authority officials, may further complicate and add to the uncertainty faced by decision-makers. The FHA in their regional guidelines for issuing and rescinding a public notification, provide step by step instructions for the issuing and removal process, in addition to a flowchart, and further information depending on whether the risk is low or moderate to high (49). The steps as outlined in this document are shown in Box 2. Similarly, but not as expansive, the NHA in its Guideline for Boil Water Notice Decision Process, provides information to guide the decision to issue a BWA, but only for situations when given evidence for low or high E.coli or total coliform counts (50). Such supporting documentations, if standardized (to a certain extent) and implemented across BC, may alleviate some of the problems with inconsistency in approaches from decisionmaker to decision-maker. However, with BC's de-centralised approach to decision-making, it is important that such documentation allow for enough flexibility in allowing the management of each unique water system appropriately and optimally on a case-by-case basis. 
Box 2: The process for determining if a public notice is required and for rescinding a public notice (adapted from the Fraser Health Authority's Guideline for Issuing and Rescinding a Drinking Water Public Notice)

\begin{tabular}{|c|c|}
\hline \multicolumn{2}{|r|}{ ISSUING A PUBLIC NOTIFICATION } \\
\hline STEP 1 & $\begin{array}{l}\text { Health authority official notification } \\
\text { The health authority official is notified of some risk to drinking water, commonly via lab notification } \\
\text { or the water supplier. }\end{array}$ \\
\hline STEP 2 & Notify Drinking Water Manager and the geographic MHO \\
\hline STEP 3 & $\begin{array}{l}\text { Consult water supplier } \\
\text { To gather information on the nature of the threat, the case and any actions planned to mitigate or } \\
\text { correct the threat, according to the water system's Emergency Response and Contingency Plan }\end{array}$ \\
\hline STEP 4 & $\begin{array}{l}\text { Water system file review } \\
\text { A number of factors relating to the water system characteristics should be reviewed }\end{array}$ \\
\hline STEP 5 & $\begin{array}{l}\text { Confirmatory testing } \\
\text { The water system should be re-sampled }\end{array}$ \\
\hline STEP 6 & $\begin{array}{l}\text { Consultation with the Drinking Water Manager and geographic MHO } \\
\text { Preliminary determination of the risk posed to consumers (whether low or moderate to high) }\end{array}$ \\
\hline STEP 7 & $\begin{array}{l}\text { Site investigation prior to a public notification being issued (where applicable) } \\
\text { To verify and collect additional information for risk assessment }\end{array}$ \\
\hline STEP 8 & $\begin{array}{l}\text { Issuance of a public notification } \\
\text { Decision to issue and type of public notification to be made with Manager, geographic MHO and in } \\
\text { consultation with water supplier. Water supplier to activate Emergency Response and Contingency } \\
\text { Plan, submit a copy of the notice to health authority for review and indicate how the notice will be } \\
\text { communicated to consumers. }\end{array}$ \\
\hline STEP 9 & $\begin{array}{l}\text { Notification of health authority staff and support agencies affected by the notice } \\
\text { Such as clerical staff who update the health authority's website with information about the public } \\
\text { notification }\end{array}$ \\
\hline STEP 10 & $\begin{array}{l}\text { Confirm notification has been issued } \\
\text { On the same day the notice is to go out, the health authority official should verify by appropriate } \\
\text { means that the notice was issued by water supplier }\end{array}$ \\
\hline \multicolumn{2}{|r|}{ RESCINDING A PUBLIC NOTICE } \\
\hline STEP 1 & $\begin{array}{l}\text { Water supplier to notify health authority } \\
\text { Health authority official to review relevant factors and criteria for the purpose of removing public } \\
\text { notification, with water supplier }\end{array}$ \\
\hline STEP 2 & $\begin{array}{l}\text { Health authority official to consult MHO and Program Manager } \\
\text { Present information indicating the condition for a public notice no longer exists }\end{array}$ \\
\hline STEP 3 & $\begin{array}{l}\text { Authorise rescinding of the public notice } \\
\text { Contact water supplier and review wording of rescind notice. Notify staff and support agencies. }\end{array}$ \\
\hline
\end{tabular}

The IHA, in their Drinking Water Public Notification Policy and Guideline Recommendation, provide a risk evaluation checklist for decision-making teams to review for consideration when deciding which form of public notification to issue on a water system, as well as a checklist for when deciding to rescind the notice (51). Also in this document are details on what actions need to be implemented once a specific public notice has been issued (51). In 
2006, the IHA developed the Turbidity Education and Notification Campaign, in response to inconsistent messaging to the public about their drinking water, especially during spring runoff time (when turbidity tended to increase) (52). The goal of this campaign was to educate water consumers, particularly high-risk groups such as infants and elderly, about turbidity and the associated health risks and notify them of elevated turbidity levels (52).

A turbidity index rating system was created, where water with turbidity less than 1 NTU is considered "good"; turbidity exceeding 1 NTU, but less than 5 NTU is "fair" and requires the issuing of a water quality advisory; and water with a turbidity greater than 5 NTU is "poor" and requires a BWA to be issued $(52,53)$. As part of this initiative, a toolkit was developed for water suppliers within the region, containing ready-to-use material, such as news release and notification letter templates, to use for notifying their consumers in a professional, timely, and consistent manner (53). The effectiveness of this method of issuing public notifications is unknown, but some early criticism from water suppliers included: complaints of additional workload; downloading the decisions about risk to the lay public themselves; the possibility of inundating the public with information and creating message fatigue and public complacency; and the need for public notifications to consider other factors in addition to turbidity and not solely turbidity (52). Further, a government report on turbidity and microbial risk in drinking water stated that a water quality index is not a scientifically feasible and practical option as an algorithm for issuing public notices, as the need for judgement based on various factors, in addition to the triggering event, would lead to better decision-making (10). 
Determining if it's warranted... it's not always clear cut. We have a complication of presumptive results from the lab at times. They're not certain, so you have to decide to take the step or not or whether to wait.

- A Drinking Water Officer

We're not going to issue [a boil water advisory] unless we see that there's a risk. We're not going to waste everybody's time. If you start issuing advisories just for the sake of doing it - if you think there may be a questionable [water sample] result [and] if it only took one result that was bad [to issue an advisory] you get message fatigue... When people see that they think - "they don't really mean it this time". There has to be a reason.

- An Environmental Health Officer

The challenges are trying to ascertain what would be considered an acceptable risk or conversely an unacceptable risk in terms of issuing an advisory. There are criteria in the Drinking Water Officer's Guide and we use that, but there's a lot of interpretation that goes along with that, so we tend to be conservative with our risk acceptance. In a sense if we don't issue an advisory we're accepting risk on someone else's behalf... We tend to not want to do that. We tend to issue advisories fairy liberally. That is a little bit of a challenge, [since] there's nobody overseeing the boundaries of that. Because of that independence that we have, we tend to issue advisories on a fairly liberal basis and that leads to a relatively high number of advisories.

A Drinking Water Quality Specialist

The 2000 Walkerton outbreak and the 1998 Sydney water crisis are prime examples of outcomes that may result when: 1) a decision is made not to act when it is truly needed; and 2) a decision is made to act when it is not truly needed (16). There were warning signs at Walkerton that were ignored for 20 years, which ultimately led to the tragedy (16). It is therefore, generally thought valid for decision-makers to take the precautionary approach to public health decisions in order to avoid such severe consequences. However, there are inevitable consequences to taking action when not required either. In the Sydney case, a BWA was issued based on erroneous water monitoring results $(16,54,55)$. As a result, tens of millions of dollars from public funds were spent on a situation where the public was not in any danger of being harmed (16). There are also disadvantages to issuing BWAs - or liberally issuing BWAs, which include: message fatigue, creating unnecessary panic among the public, the potential for the public to lose confidence in their water system, increasing the risk of burn injuries among the public from boiling water, and the possibility of creating a "cry wolf" situation, where the public may not take future BWAs seriously (16). Many of these are further discussed and brought up throughout this report.

Making decisions within a short time frame is a challenge, especially when decisionmakers are given little information to work with and the outcome of the decision may have public health implications (10). Often times, health authority officials and water suppliers are 
faced with water sample lab results which don't clearly indicate that there is a definite risk to the drinking water. Decision-makers are left with the dilemma of taking immediate action based on presumptive results or submitting more water samples and waiting another 24-48 hours for the water sample results before making a decision. A number of health authority officials mentioned the 'Friday 4:30 pm' scenario - when a possible BWA trigger is identified late Friday afternoon, and the official is left to decide what to do at that point when evidence of threat to drinking water is insufficient, the water supplier may not be available, colleagues have already left for the weekend and further sampling cannot occur until Monday. Should the decision-maker err on the side of caution and immediately issue a BWA for the sake of precaution or should s/he wait for more definite evidence that ensures the threat is real? Dealing with such uncertainty is probably the biggest challenge health authority officials and water suppliers face, as it was raised by many participants. To issue on the basis of precaution, even when the BWA is not warranted, may create panic among the public and unnecessary harm to the public's confidence over the water system. The over-issuing of BWAs at a water system may lead to message fatigue - the public will not comply with the message recommendations and the BWA would lose its value.

The decision to issue a BWA, especially under uncertainty can be very subjective. Some health authority officials prefer to issue a BWA based on any slight trigger, whereas others would hesitate to issue unless sufficient evidence is available and would issue as a last resort. Some health authority officials provided examples of past BWA issues by colleagues in and outside of their health authority, where they did not agree that it was warranted. How much evidence is enough evidence for issuing a BWA? In the current process, it is a judgement call left in large part to the decision-makers' discretion, after taking into account criteria that they have deemed appropriate. Their personal beliefs, perceptions of perceived risk, past experiences, and history with the water system all come into play. A report by the Technical Advisory Committee indicated that a universal index cannot be created for decision-making with regards to BWAs and other types of public notifications (10). Informed decisions are best made on a case-by-case basis after considering a number of system specific risk factors $(10,41)$. 
We have an idea ahead of time; it's not normally a surprise when something happens.

- A recently retired health authority official with 36 years of experience

I've been with some water systems for 14 years... If we have a positive sample I'd have a pretty good idea why it happened. [I'd] understand the operator, [I'd know] where the faults are in the system, [and] the quality of the water.

A health authority official with 20 years of experience

Sometimes decisions are not made by experienced [officials]. Erring on side of caution is ok, but [we] need to avoid unnecessary issuing of boil water advisories under situations that didn't require it or [when] there was not enough evidence to show unsafe water. It's unnecessary harm over the confidence of the system.

- An Environmental Health Officer

The amount of experience health authority officials have with BWAs and the competency or amount of training water operators have may influence how the decision is made or whether a BWA is issued, respectively. A more experienced health authority official may not find the need to consult with other individuals within the health authority as much as someone with less experience. S/he would also have more years of experience in working with the water supplier and the water system under question and would know what to anticipate with regards to the need for a BWA and whether compliance can be expected from the water supplier. On the other hand, less experienced health authority officials may be more likely to issue BWAs under uncertain conditions, as was raised by some health authority officials. Based on interview data pertaining to discussions on how much evidence is enough for the issuing of BWAs, when comparing health authority officials with less than 15 years of experience with drinking water or drinking water programs, to those with more than 15 years of experience, the former group mentioned the need to "err on the side of caution" or "take a proactive approach over any potential concern" and that "concrete evidence need not be present" more so, than the latter, more experienced group. The observation, however, should be interpreted with caution as the sample size was low to allow for a fair comparison between the groups.

A lot of the time, when doing inspections, I ask water operators to draw a schematic of the system and they don't know where the pipes are.

An Environmental Health Officer

If a water system lacks a trained operator, there may be a higher tendency for a health authority official to issue a BWA on the system versus one where the operator is well-trained and competent in maintaining the water system, including routinely submitting water samples for testing. There is no requirement for small water suppliers to be formally certified in $\mathrm{BC}$ and it is under the discretion of the DWO to decide whether a supplier requires certification or not. 
Common certification program for water suppliers in $\mathrm{BC}$ include the Environmental Operators Certificate Program (EOCP), training programs offered by the BC Water and Waste Association and courses offered by institutions like Thompson Rivers University. The DWPR, however, specifies that an EOCP certified water supplier is considered qualified to operate, maintain or repair a water system (48). Regardless, to protect public health, attention to the training and certification of small and remote water system suppliers is crucial (12).

Small water systems with a high turn-over in water suppliers and/or operating staff are problematic, as are those where longstanding, highly-experienced water operators are retiring. The challenge of dealing with an increasing population of retiring water operators - particularly in remote communities, has been documented in other literature (12). The opportunities for retiring water suppliers to pass on their historical operational knowledge on the water system becomes limited (12). Finding qualified staff or replacements in remote locations is challenging (12). With the change in water supplier, the need for re-establishing a new relationship between the health authority official and new water supplier becomes important. The importance of a good working relationship will be discussed later. With staffing changes, as when a new health authority official or a new water supplier or operator takes oversight of a water system, the value of good record keeping is realised. Many small water systems do not keep historical records of the water system, making transition to new operating staff difficult (12). While conducting an in-person interview of a health authority official, it was observed how much the officials depended on records to answer some of the interview questions. Some records were poor and incomplete, and there were information gaps that the health authority official had no way of filling. Without thorough knowledge of a water system, the decision to issue a BWA, by health authority officials and water suppliers, inevitably relies on a much smaller set of information.

UBC is closer to San Francisco than we are to our branch office... It's difficult for us to get around our area [and] it is a problem geographically. When we go to the branch office it takes us 10 days. We do all the water systems all the way up and all the way down, but it takes three days driving plus all our stops. Three days one way. It's a different scale of things up here... Certainly geography's a challenge for Northern Health that the other health units don't have to the same degree. 
A long-term goal of health authority officials is to inspect each water system at least once a year (20). In 2000, only $24 \%$ of all BC public water systems ${ }^{3}$ were inspected at least once during the fiscal year (20). Data on the number of drinking water staff employed that year was not available. This goal, however, is not being met as there is insufficient staffing. Some officials have responsibilities for other (non-drinking water) programs and therefore tend to focus on high priority water systems, like those that impact the largest number of consumers (20). Another challenge to regular inspections that health authority officials face - and that was revealed in this study - is the challenge of geography, especially in northern BC. Because of the remote locations of many small water systems, commuting to such sites can take days. The geographical locations of water systems may also delay the testing of water samples, as in some cases, water samples must be transported from the sampling site to another location prior to being shipped to labs for testing, since onsite or local water testing facilities are not available near rural water systems. Getting contractors, workers or equipment for the construction or upgrade of a remote water system may also be delayed due to such reasons.

I have unsuccessfully tried to get more testing facilities in Chilliwack. We need more testing facilities [here]. My wife drives 18 kilometres to the Fraser Health Authority to drop samples off Monday morning. From there it goes to Abbotsford and then it's sent to Burnaby for testing. It's a waste of energy and resources. We get the sample data 3-4 days later by telephone after the case.

- Water System Operator and Manager of Water User's Community

With the advancement of remote sensing and data transmission technology, perhaps a better long-term goal of BC Public Health Protection could be to implement such technologies at water systems (especially remote ones), such that continuous, real-time data on water quality factors are available to health authority officials, reducing the need for inspections of water systems and providing better information on water quality to base public health decisions on. Not only may this allow for the collection of complete data on water quality and reduce the ecological footprint from long distance commuting to remote and rural sites, but this initiative would also address one of the information gaps, as identified in an report by the Provincial Health Officer in 2007, on the need for province-wide, real-time tracking system on water quality data (6). However, the logistics of implementing this across $\mathrm{BC}$ would be challenging,

\footnotetext{
${ }^{3}$ An estimated 3,016 water systems as of March 31, 2000
} 
and would likely take many years, especially given the current impoverished state of many small water systems.

\subsection{Public Communication of Boil Water Advisories \& Public Compliance}

Although water suppliers are deemed responsible for issuing BWAs, as per the DWPR, in most cases and especially in small water systems, it is the health authority officials who initiate the process and issue directives to the water suppliers to take appropriate action starting with communicating the BWA to the system's consumers. The majority of water suppliers are aware that the responsibility of informing their consumers about a BWA is primarily theirs. The two most common methods used to communicate the advisory to the public in this study were: 1) inperson, including door-to-door canvassing and announcements at gatherings, like in meetings or at camp or resort registration and orientation (61\%); and 2) public postings, such as signage at water faucets $(55 \%)$. Not all water suppliers had access to or made use of templates for informing the public about the BWA. Water systems with an emergency response plan in place had ready-to-use templates of signage available. The Drinking Water Officer's Guide also provides generic templates, as do some health authority specific guidelines $(8,49)$.

I don't communicate it as a boil water advisory. I tell the public that the water is polluted, the government is on my back [and] this is what we have to do [to end the advisory]. Each house is dealing with this their own way. Some are using roof-water in addition to community water, some are on [a] point-of-use or point-of-entry [device]; [some] own a refurbished well at their property, etc.

- Water supplier of a Water User's Community Water System

There are no standardized ways of communicating a BWA across BC, nor any consistency in the type of information that is provided to the public. Rather, in most scenarios, health authority officials and water suppliers tailor-make the BWA message according to the water system under question. To some extent this is appropriate, given the diversity of water systems in BC. For instance, in water user group communities under long-term BWAs, rather than the advice to boil water before use, many water suppliers encouraged the consumers to invest in the use of alternate treatment, such as the installation of point-of-use/point-of-entry devices. In such scenarios, water suppliers have complained that the label "boil water advisory" does not fit them. 
A number of studies have examined the public's perception of drinking water quality and their water consumption patterns and preferences $(14,56,57)$. In the BWAs investigated in this study, all health authority officials and most water suppliers recommended boiling water prior to use. It would be interesting to see what proportion of these water consumers actually boiled water during the BWAs, rather than use bottled water or some alternative source. Studies have indicated that communities under a long-term BWA are more likely to use bottled water over boiling tap water (22). One BC study found higher bottled water use in East Kootenay (IHA) than in Vancouver (14). This was attributed to the region experiencing a high number of BWAs that may have consequently resulted in the negative perception of tap water among the public (14). In the NHA, conversely, it was found that bottled water use, in addition to in-home treatment devices use, was lower than that in Vancouver (14). Perhaps this is due to the majority of the population's belief on the pristine nature of their drinking water supply.

Use of clear wording and the inclusion of specific recommendations in BWAs may improve public compliance. One study mentioned that the clear indication of risk reduction measures in the BWA message, such as using boiled or treated water for brushing teeth or washing fruits and vegetables, could be helpful (26). Specific advice for vulnerable populations should also be included (26), but such information was lacking in the BWAs investigated in this study, as was information on how long the BWA was expected to last and what corrective actions were being taken. Another study found that the public affected by a BWA wanted to know when the BWA was anticipated to be lifted and updates informing of any progress or actions done to correct or address the BWA triggering event (18).

Health authority websites vary in the amount of detail and type of information that is available for the public on BWAs. All five of BC's health authorities provide an updated list of BWAs. Of all health authorities, NHA and FHA provide the most comprehensive information about their water systems, via facility information, inspection history, inspection reports and water and chemical test results (4). All, but VCHA, had a link to more information about BWAs (ie. what they are, when they are issued, what to do during a BWA) on the webpage with the listing of current BWAs (4). All health authority's online lists of BWAs provided at least the name of the water system and the BWA effective or issue date. VCHA and IHA also provided the reason for the BWA. As internet use is increasing, better public access to clear, 
understandable and adequate information about their drinking water will become more and more essential. One water supplier utilised a Facebook group as a means of notifying its consumers about the BWA.

Communication with the operator: We call, we leave a message. It might be 3 or 4 days before they get back. If it's urgent we have to drive out there or use another means, such as asking the RCMP to pop in and ask them to call us - because of service not for enforcement - but when it's urgent, we don't have a choice. There are times when we are unable to contact the water operator. We have to drive up there and knock on [the consumer's] doors ourselves (to inform the consumers about the BWA) and this is not because the water operator was not willing, but because he couldn't get back in time from the bushes or camp.

A Drinking Water Leader

Water suppliers of small water systems, in particular, are not easy to contact at times, and so health authority officials are forced to make a number of phone calls before contact is made. This was a challenge faced during the recruitment of water suppliers in this thesis as well. One specific water supplier's phone number suddenly became 'out of service' during attempts to contact him. Health authority officials have faced circumstances where the water supplier's contact information has changed, without their knowing. In some instances, updates to the water supplier's contact information are only possible once an onsite visit to the water system is made by the health authority official. Related to this is the health authority's lack of information on the public being served water from specific water systems. While trying to obtain the contact information of members of the public being served water from specific water systems on a BWA (as part of an excluded segment of the thesis), it was found that only the water suppliers hold this information. If such is the case, does this indicate that the health authority would not be able to assist a member of the public who was to call them for information about their drinking water, but did not know which water system they receive water from?

[We have] busy lives. [There is] not one source of information anymore. We each get different sources of information... Making sure what method and format is going to capture 100\% of the people who need to know [is a challenge].

- $\quad$ An Environmental Health Officer

Effective communication with the public is getting the [boil water advisory] message out to the different facets of the public. We're dealing with different parties: people who listen to media, people who read signs and people who don't get out to get those various messages. You've got your various age groups who take messages differently. [For] effective communication [you need] clear wording [and] multiple points of communication... We need a feedback mechanism, which we don't have, to see how effective that communication was.

- Water Specialist 
The outcome of negligence in informing the public about risks to their drinking water and inadequacy in the methods of communicating BWAs was realised and documented as early as 1930 (35). Since then, a vast amount of literature has emphasised the importance of risk communication through various channels and approaches, particularly when communicating public notices of water quality $(10,18,25,26,58,59)$. For effective communication, various factors specific to the target audience need to be considered $(10,58,59)$.

The water supplier, being a part of the affected community (in most cases), has an important role in conveying the BWA message as s/he would know the more about their consumers than the health authority - this is especially true for small water systems. The water supplier's personal beliefs about the water quality and tone by which s/he verbally conveys the BWA message to the consumers can influence the public's perception of risk and consequently public compliance. If the water supplier doesn't believe the BWA is necessary, how will s/he convince the public to heed the BWA's recommendation to protect their health?

You are leaving it at the hands of the water operator to notify the users of that system. Unless we physically go out and actually have a look to see that notices have been provided, people have been informed... There's no 100\% certainty that everybody's been informed - that's the weak link here.

An Environmental Health Officer

There's no verification that [the consumers] are receiving the message or if they care. There's no emotional impact of the advisories. You want people to understand that there is a risk. Tone can be a huge indication of risk. If I come to your door and say - "there's some stuff going on, maybe you should boil your water... Whatever, I'm drinking it. It's fine, I'm healthy as a horse" - it downplays the message that was embedded. You can have a message but it's how it's delivered that's going to have an impact... and there's no control over that. We just can't be there. We don't have the resources to be there. We can have a meeting [with the water supplier] to talk about it. Not being able to interact with our clientele (the public) [is a challenge]. Ultimately we're working for the people who are drinking this water and not the water supplier.

- An Environmental Health Officer

On a similar note, there is the challenge of changing a community's perception on the safety of their drinking water. Targeting all members of the public affected by a BWA, itself, is a challenge, but ensuring that the public is complying by following the advisory's risk reduction measures are made even more difficult in communities consisting of non-believers. One health authority official narrated an experience s/he had with a community that considered their water supply a 'fountain of youth'. When a BWA was issued on their water system, community members tore down the BWA signs. Such strong opinions and beliefs about drinking water supplies are common in rural communities in $\mathrm{BC}$, where there are a large number of people who 
oppose chlorination, perceive their water as pure and pristine and are defiantly trying to keep their drinking water supplier from being chemically disinfected. One study on a noncompliant town under a boil water order, qualitatively described the conflicts between the townspeople and regulators in their opposing beliefs and opinions on risk perception (28). If the targeted population is not adhering to the BWA's advice, then the issuing of a BWA becomes a wasted effort (41). In such situations, health authority officials and water suppliers must be careful, as studies on risk communication indicate that when the public receives advice (such as to boil water or chlorinate) which they oppose, they may lose confidence in or begin to distrust the provider of information (the health authority or water supplier) (59).

When you have a situation where people have been consuming water already for long periods of time and we put a boil water notice, it doesn't have credibility for the people - '[I've] been drinking glacier fed river water all my life and I've never been sick. Why are you telling me it's not healthy to drink?' In some situations people will move into an area like that and will ask neighbours about the advisory and be told'Ignore it, I've been drinking it all my life, there's nothing to worry about' - and then they get sick. - $\quad$ A Drinking Water Officer

Risk communication is the biggest challenge. A lot of people up north and on these small water systems really do not like chlorine in the water - or fluoride for that matter. Trying to get them to understand [the] differential risk they are facing from the disinfectant-by-products versus risk of infection from pathogens in the water is a challenge

- $\quad$ An Environmental Health Officer

There's a lot of people [whom] we tell that they should boil [their water] and they simply tell us that they've been using the water for 15 or 20 years and they've never gotten sick. People chose to do what they chose to do regardless of our expertise and advice.

- $\quad$ An Environmental Health Officer

[The health authority] wants treatment at source and that would cost well over $\$ 10,000 \ldots$. It's just families getting water from [the source] and no one has ever been sick. They are very opposed to chlorine. The water is just wonderful water and there's never been any fecal coliform in it.

- Member of Community Water System

A number of health authority officials have emphasised the value of good communication and a good relationship, involving trust, with the water supplier. A relationship in which the health authority official and water supplier agree on the need for a BWA or where the health authority official can convince the water supplier, based on a relationship based on mutual understanding and concern for public health, is likely to lead to better management of the water system than one which forces the water supplier to issue a BWA. Maintaining good relations, however, can be a delicate task. Health authority officials don't want the water supplier to feel incompetent or non-diligent when they are told of the need for a BWA, especially since some 
water suppliers don't perceive the label of being on a BWA positively. Some water suppliers take pride on their water system and take it personally when a BWA becomes required. The reticence of water operators to issue BWAs is understandable, as they fear the consumers may lose faith in their systems, and it is a real possibility having been documented as one of the cons of issuing BWAs (41). However, whilst a BWA may weaken the public's confidence in the water system, it may enhance the public's awareness of their water supply and water system (41) and become a catalyst that triggers further remedial action and improvements to the water system.

If we know the operator out there and have worked with him for a number of years and we know the person - know that they are very good and good with communication and will get the notice out - we got a little trust going on there... [The] weak link is the operator-getting that notification out. It's not up to us to do that, it's up to the operator.

An Environmental Health Officer

Responsible and irresponsible households [are] in the same pot but they are seldom "bad".

- Water supplier at a Water User's Community water system (In reference to how all households under a water systems are perceived as "bad" or "irresponsible" with the boil water advisory tag on the system, when most individual households are complying through the use of point of use/entry devices.)

Message fatigue is a major concern of health authority officials and water suppliers, and a negative consequence of longstanding or frequently issued BWAs. In relatively well-managed systems, where BWAs are scarcely issued, when something goes wrong triggering the need for a BWA, the public takes notice and tends to comply. The BWA that took place in Metro Vancouver in 2006 is an example of such a case, as it was the first BWA in the history of the water system (43). At water systems where BWAs are issued in an on-again-off-again manner or where BWAs have been in place for years, compliance suffers as the public stops taking the BWA seriously. A number of studies have shown that compliance decreases the longer a BWA is in place $(12,22,27,30)$. Most studies were based on short-term, temporary BWAs in outbreak situations. If compliance suffers in such scenarios, then it is worrisome to think what the reality of public compliance must be in longstanding, months-to-years-long BWAs, as there are in BC. This concern has also been raised in past government reports and the seriousness of this matter is re-exemplified in this study (6). 
People are so used to seeing notices and advisories that some of them just ignore it.

- Water System Operator

In some systems [public compliance] is a concern. For instance [at Water System A], where there are multiple boil water advisories [issued], people get used to them and stop listening to them or heeding them. [At Water System B], it's their first one. They got that notice and they didn't freak out but they were shocked and took notice of it. If you get a boil water advisory every month or 2 months, you get complacent and they're not very important to you.

- A Drinking Water Officer

[The] longer [a boil water advisory] goes on, the less people will care about it.

- Environmental Health Officer

If people are making an informed choice to drink water even if it's unsafe, I don't have a problem or concern with that. People can choose to be hit by a car, or eat contaminated food. As long as its informed decision, I don't mind whether they comply or not, because they have a consequence then.

- $\quad$ A Drinking Water Quality Specialist

Public compliance is, therefore, a huge concern that dictates the overall value and effectiveness of issuing a BWA. Although many health authority officials indicate that the primarily goal of the work they do is for the public and maintaining their health, ensuring public compliance towards BWAs is something that they can rarely do anything about. Therefore, water suppliers have a greater responsibility and can do relatively more than health authority officials can to encourage compliance. Health authority official and water suppliers' perception of public compliance may be a reflection of this: a lot of the health authority officials do not know whether the public is complying or not and there is no practical or absolute way for them to determine this. Efforts are therefore best put towards producing a clear, effective BWA message and ensuring that the message is disseminated via numerous methods to capture the entire population affected. Particular care is needed for water systems serving populations with a high turnover rate, such as those serving tourists or seasonal vacationers. For longer running BWAs, regular reminders to the public are essential, but not frequently done, as it's often assumed that everyone already knows.

\subsection{Actions in Effort To Rescind Boil Water Advisories}

At the time a BWA is issued, decision-makers should determine when the BWA may be lifted and what criteria will determine this. This recommendation has been cited in a number of reports $(10,36,37,41)$. Establishing removal criteria may be difficult in long-term BWAs, where the advisory might be used as a band-aid to action or to treatment, or for BWAs issued as 
precautionary purposes, as there is no concrete solution or clear corrective action to take $(10,41)$. Such criteria should be created with the consultation of water suppliers, nevertheless, to help indicate when the BWA can be removed. One study recommended a step-wise BWA, that can be scaled up or down, for situations where concrete solutions or criteria cannot be agreed upon and developed (41).

In the investigation conducted in this study, health authority officials and water suppliers did have some benchmarks or criteria in place to help dictate when the BWA is ready for removal. Actions required for the removal of the BWA included: 1) the installation and introduction of appropriate water treatment and/or disinfection; 2) the repair or replacement of malfunctioning water treatment and/or disinfection systems; 3 ) the creation and implementation of a water system operation and maintenance plan; 4) cleaning, disinfecting and flushing the water system; 5) fulfilling the criteria of having all households on a community water system on a point-of-use/entry device; or 6) the development of a source protection plan. Ultimately, the BWA would be removed upon evidence that the water is of satisfactory quality, as indicated through negative water sample results.

[Boil water advisories] are the best tools that we have for a variety of reasons. One, of course, we notify the public right away so at least they have an opportunity to respond positively at their choice. The other is a reminder to the operator that a change needs to occur. [Boil water notices] are posted publicly; everybody gets the notice... [and] people are really well aware of it. Of course we're doing the follow-up. We keep going back and meeting with the operator to work on a resolution. We just don't issue a boil water advisory and kiss it goodbye. We keep doing the follow-up until it's resolved.

- $\quad$ A Drinking Water Leader

Some systems have been on boil water advisories for 20 years. What happens to that message after 20 yrs? Is there a message anymore? It's weakened and it's not news anymore. Boil water advisories are better for immediate impact and hazards and that have timelines for corrections attached.... Water systems are complicated, not like a fixed facility where you can yank their business license or food permit. It's more hazardous for people to not have water when it's dirty. You can't shut off their water supply [and] you don't want to fine these places. Then they'll have to pay a fine on top of boiling water.

- An Environmental Health Officer

The biggest obstacle to the removal of long-term BWAs is money. Small water systems, especially, lack funds for the addition or improvement of infrastructure. For example, a water system on a poor water source may need to incorporate multiple barriers in the form of treatment for the water to be of satisfactory quality; with the need for more barriers, is the need for more financial investment into the water system. Some small water systems are closely tied to the 
economy. In this study, water systems linked to the tourism industry, fishing industry and lumber industry were identified and investigated. Two water systems, that served resorts and lodges, were undergoing closure and bankruptcy at the time of the study.

There really are no issues for issuing [boil water advisories], the issues come into place with removing them, because of financial roadblocks. The cost of treating or putting treatment in place to solve the problem is very costly and that's the problem -it's the removing. The only reason why these advisories are existing is because these water systems don't have money to replace infrastructure or put in treatment.

An Environmental Health Officer

The biggest obstacle to getting treatment on these water systems is financial. It's not a lack of will - it's finance. The small water systems; the private ones; the ones that are not part of the municipality that can raise taxes. If they had the money they would do it. [There is] nothing out there that would give them money to help them. There is nowhere they can apply for grants.

- An Environmental Health Officer

The problem small water systems face with the lack of funding is one that has been known and documented $(12,13,42)$. Due to the small number of consumers served water by small water systems, the spreading of the cost of operation, water testing, maintenance and longterm planning becomes difficult (12). Some water systems governed by an elected board are limited in their ability to raise water prices, as it tends to lead to consumer outrage (12). Infrastructure grants are not accessible by non-government owned water systems and small water system owners are without options on how to fund changes or upgrades they need in order to have BWAs lifted (42). A number of health authority officials mentioned the financial roadblocks that small water systems face and have reiterated the need for the government to offer financial support or consider other solutions, such as incorporating smaller water systems under the municipal system or create a unique, better fitted governance structure.

Small water systems are often struggling with compliance to safe drinking water legislation. Not all water suppliers understand the standards and feel that the requirements are too stringent and not fitting for their system (42). At present, all public water systems - whether serving a resort of 20 people, a lumber mill with 5 employees, a water pump at a provincial park, a community of 8 households or a metropolitan population of 1 million - fall under the same blanket drinking water regulations. Regardless, water suppliers do want to move towards the removal of BWA, but many are simply limited in resources. There are no funds to cover the costs of infrastructure upgrades or improvement; remote and rural systems are isolated and are 
located far from water labs; water suppliers are busy people who are juggling many roles, as an entrepreneur running a corner store, for instance, and many responsibilities, such as tending to sick family members or running a poultry farm - in addition to the role and responsibilities of a "water supplier", and don't always have an abundance of time. It was interesting to observe that a majority of the health authority officials show understanding and sympathy for the water supplier's predicament. Health authority officials try what they can in accommodating the water supplier's interests and show patience, and at times leniency where deemed appropriate, while working with them towards solutions. Each BWA is, therefore, uniquely managed by health authorities with consideration of the water system and water supplier's story, as much what they are required to enforce in the regulations.

\subsection{Other Toils, Troubles \& Thoughts}

It's precautionary only. It's sort of like after the horses have run out the door, you close the barn doors. It's not really a diagnostic tool [and] not to treat the water system in place. It's a last resort.

- An Environmental Health Officer

Are BWAs an effective tool? The majority of health authority officials and water suppliers tend to think so - but only when intended for short-term, temporary, precautionary purposes and communicated effectively. Some water suppliers, however, believed BWAs were of no value since some have been in place for so long that no one pays attention to them anymore and others believed they were issued by the health authority simply for the sake of "evading liability".

You can't come up with one treatment process that goes with every [water system]. That would be a disservice to the complexity of water. There is no one answer for everything around here. One size does not fit all.

- $\quad$ Environmental Health Officer

If anyone in town heard me say this I'd be hung: chlorination. As far as I know, it's the only way of disinfecting the entire line - every nook and cranny. With UV, it only gets any bacteria that [are] in front of it.

- $\quad$ Public Works Superintendant (On whether s/he thought any form of treatment or disinfection should be mandatory)

If the lack of water treatment or disinfection is a major trigger for the need for BWAs, should water treatment and/or disinfection be mandatory for all public water systems in BC? 
Health authority officials and water suppliers did not support the mandating of treatment and/or disinfection in all water systems, and rather agreed that the best approach would be to evaluate each system's unique requirements individually, as there are water systems that serve good quality water and don't need treatment at all - Chilliwack's water supply being a good example. Groundwater, for instance, in most cases doesn't need filtration as water naturally filters while percolating. Similarly, not all deep wells require disinfection. However, many were in favour of requiring all surface water supplies to be filtered or disinfected, the latter being a requirement as per the DWPR. Some mentioned that chlorination could be a cheap and effective means of disinfecting water supplies. Health authority officials and water suppliers realise that the decision to mandate treatment and/or disinfection in all water systems in BC would be an impractical one. There are not enough resources to put in treatment where it is most needed today and investing in treatment where it is not required would be an unwise use of resources.

There could be some room for non-regulatory approach to these ultra-small systems and dealing with them on a voluntary compliance basis - mindful of resources becoming scarce and budgets getting tighter. Is it in public interest to regulate these ultra-small systems when in fact many other jurisdictions are not? If you've got only finite resources [and are] spending those resources over too large of a capture area, it may be better served to refine, to prioritise, and do a better job on focusing on those larger systems and deal with smaller systems by some other means such as voluntary compliance or [some] educational approach. [It's an example of] diminishing returns. As you get to smaller system-you have more and more challenges with water quality and smaller and smaller proportion of population affected. [From a] population health perspective, there probably is some limit to where we need to draw the line at it.

- A Drinking Water Quality Specialist

In BC, we look down to two houses on a single source [to be considered a public water system]. I don't know if that's a model widely adapted elsewhere. As we get to smaller and smaller [water systems] we spend more and more resources trying to invigilate requirements on [them], that may not want in the first place.

A Drinking Water Quality Specialist

The vast majority of problems with BWAs affect small water systems and consequentially affect a small proportion of BC's population. A lot of health authority officials realise this and emphasise that some alternate form of governance needs to be instituted for small water systems. A number of health authority officials have confessed that they have trouble with the definition of a water system as anything serving more than a single-family home, and would like to see a more restrictive definition that limits what falls under the threshold of regulation and under their jurisdiction. The Provincial Health Officer, in his 2000 annual report, acknowledged this suggestion, but concluded that any water system, regardless of the size, needs to be under public accountability and have their consumers' health protected from waterborne risks (20). In 
this report, it was recommended that other governance models be explored, such as the US's strategy of applying different drinking water standards according to a water system's size and type (20). A system which limits the growth in the number of small water systems, such as through limiting water permits for new systems or encouraging small systems to amalgamate with pre-existing municipal ones, may be better options.

[It's good that] Walkerton happened because it helped us do the work we want to do - which is protect the public... It's too bad it took dead bodies to wake up. It's hard to make a case until dead bodies show up to get politicians' attention. [I'm facing a] problem with hot tubs in my area... Unfortunately someone will have to die in a hot tub before we get things back on track - that's how it is in prevention program.

An Environmental Health Officer

Operators take [the safety of drinking water] much more seriously and are quite concerned about liability. Then the flip-side to it is - often operators will blame any requirements put on them as a reaction to Walkerton. They don't see it as a specific issue for them. [They think] they're being painted with the same brush.

- $\quad$ An Environmental Health Officer

We got a blanket boil water advisory shortly after Walkerton. Politicians became panicky [and] they didn't know about water quality. As soon as they realised that they are liable and panicked, these advisories were coming out left, right and centre. They went overboard and had extreme drastic measures taken.

- $\quad$ Secretary at Strata Water System

What changes have health authority officials and water suppliers witnessed as a consequence of the Walkerton, Ontario outbreak? Since 2000, there has been an increase in the attention given to the importance of ensuring safe drinking water. Provincial regulations improved and numerous drinking water programs developed as a result. Provincial funding for drinking water and drinking water safety increased and some drinking water specific positions were developed. The number of trained water operators increased and the multi-barrier approach was developed. Some health authority officials have noticed a lot more compliance and cooperation from water suppliers and the public's awareness has also heightened with respect to their drinking water quality. A number of water suppliers noticed more scrutiny and caution from the health authority and interpreted this as the health authorities trying to ensure they are not liable. The Walkerton outbreak was an eye-opener; an example of how positive changes and improvements can only result when something goes wrong first. 


\subsection{Strengths \& Limitations of Thesis Study}

This study was developed to address some knowledge gaps and add clarity to how BWAs are managed in BC. It is the first study of its kind to investigate BWAs retrospectively from when they were issued to what the current situation is and what the prospects are with the BWA and the actions for its removal. The unique qualitative data has provided valuable, factual and detailed narratives.

All interviews were conducted and transcribed by the same researcher to maintain consistency in data collection. The order of interview questions did not logically follow the thought process of all participants, and therefore some participants partly answered questions in other questions. Participants provided varying levels of detail as per their comfort level and knowledge.

Participants were provided the interview questions beforehand, at the time introductory information about the study was given. This helped improve participation rates, as participants felt comfortable knowing what questions would be asked in the interview. However, this may have encouraged some participants to prepare their responses in advance of the interview and may not have allowed for an accurate determination of the participant's spontaneous awareness with certain topics - such as what types of public notifications are issuable in their health authority. This would likely have a minimal effect on the thesis overall, as a very limited number of knowledge-testing questions were asked. The majority of questions required the participant to refer to records. For some questions, such as on the decision-making process, participants were dependent on their memory (if they were present at the time of BWA issue) and records of the event. As many BWAs that were investigated were issued months to years back, it is very possible that the participants may not have been able to recall details accurately. Those who were not present at the time of BWA issue and without any detailed records may have guessed what may had happened at that time, or may have provided information as how the decision-making process is done today.

Random selection of water systems allowed for the unbiased selection of water systems in $\mathrm{BC}$ and resulted in a diversified collection of information on very diverse types of water systems. However, also due to random selection and the preponderance of small systems in the 
province, no large water systems were investigated, and the data collected in this study were most pertinent to small water systems. The small sample of water systems investigated limits the generalisation of findings to all water systems in BC or in Canada. The small sample size also

prevented the calculation of statistical significance for some tests of comparison. Chi-square tests that were conducted gave results with a warning that the approximation provided in the test may not be appropriate as the expected counts for certain variables were less than 5 .

Additionally, the results of Kappa tests may not be a true indication of agreement. This test removes any non-paired data (as in data where only the health authority official provided a response and not the water supplier, and vice versa), resulting in a shortened dataset.

\subsection{Significance of Thesis Research \& Concluding Remarks}

This thesis provided a small, illustrative snapshot of the current situation with BWAs in BC. The reasons for issuing BWAs were common to those already documented elsewhere, but this study also revealed that decision-makers facing situations of uncertainty primarily due to the lack of information may issue a BWA on that basis and not solely on the basis of evidence for poor microbiological quality of drinking water. The decision-making processes factored in varying criteria depending on who is involved and the water system under question. Criteria for the removal of BWAs were, for the most part, preconceived, but corrective actions required were delayed due to restraints in resources and technical challenges. The study highlighted the troubles health authority officials and water suppliers face with regards to BWAs: the dilemma of making decisions with presumptive evidence; the challenges with risk communication; and the problem with message fatigue and public compliance with longstanding or frequently issued BWAs. A number of issues illuminated in this study were those mainly encountered in small water systems. Solutions that look to improving the overall management of small water systems in $\mathrm{BC}$ and that provide necessary guidance to decision-makers, before, during and after the issuing of BWAs, would be of great value in alleviating some of the challenges faced with BWAs. 


\section{Implications \& Ideas for Future Research}

\subsection{The Troubles with Boil Water Advisories}

The findings of the thesis illuminate some challenges and weaknesses in the way BWAs are managed in $\mathrm{BC}$, as well as some of the problems identified in previous studies and reports. The three major weaknesses with the current situation with BWAs, as identified in this thesis, are:

1) The lack of funding available to small water systems for infrastructural upgrades or improvements (such as the installation of water treatment) identified by the health authority as necessary for the removal of BWAs, especially long-standing ones.

2) The insufficient follow-up by health authority officials and corrective action by water suppliers to progress to timely removal of BWAs, particularly long-term ones.

3) The surprisingly low proportion of water suppliers or water operators, especially at small water systems, who have the training, education and competence to manage and maintain a water system.

There is a wide gap between what is said to happen - as in regulations, policies and supporting documentations, with respect to how BWAs are managed - and what is really happening in practice. The regulations place the responsibility for BWA management largely on water suppliers. Water suppliers are deemed primarily responsible for responding to and notifying the public when there is risk to their water supply and health authority officials are deemed in charge of enforcing the DWPR. However, in reality the oversight of BWAs largely falls to health authority officials, as not all water suppliers have the resources (education, understanding, competency, funds, time, etc.) to meet the BWA removal criteria and manage the small water systems optimally. In turn, in some cases, health authority officials become overwhelmed and therefore sufficient follow-up after the issuing of BWAs does not always take place; certain BWAs become status quo over time.

In this thesis, the decision-making process was found to be inconsistent from one decision-maker to the next, both within and across health authorities. BWAs were being handled in a case-by-case basis for the most part, depending on the water system in question. This can be both advantageous and disadvantageous. 
The management of each BWA case within the context of the water system's unique circumstance and characteristics allows decision-makers to consider what is likely best for the specific water system in question. BC has a diversity of water systems. Treating all water systems the same way would be a rigid approach; there needs to be some sensitivity to context. However, at present, some of the diversity of approaches is not because of differences between systems but rather differences between decision makers. Some prefer to issue BWAs more liberally, whereas others do not. Some consider numerous factors before making decisions, whereas others do not. Some have years of experience in issuing BWAs and with specific water systems, whereas others do not. There is the potential for such personal preferences and experiences (or the lack of) to affect sound decision-making.

Inconsistent approaches can therefore be problematic from a fairness perspective. This was a concern also raised in the Ombudsman's 2008 report on drinking water in BC (3). The public should rest assured that their drinking water is being managed in the best possible way, regardless of the type or size of their water system or where they live in BC.

Inconsistency also creates confusion. There are many variants for the term "boil water advisory", as well as for the other types of public notifications. This variety exists within health authorities, across the province and the country. The definitions of the terms are also not consistent. For instance the term "boil water order" is defined differently by the province and the federal government. Confusion at the administration level may also encumber sound decision-making. Further, such confusion may extend to the public - for whom these public notices are issued.

The decision-making process for issuing and rescinding BWAs cannot be one that is prespecified entirely in procedural guidance documents and rote rules nor should it be dependent entirely on each decision-maker's personal judgement. Standardization that achieves consistency in goals, approaches and terminology within provinces and territories is needed. However, there needs to be enough flexibility to allow each unique water system to be dealt with appropriately. For this reason, leniency by the health authority, where deemed appropriate and with good reason, might be allowed, however it should not replace the need for further action 
that is required for the removal of a BWA. Rather, it should indicate that the water system is one that requires more support from the health authority, to work towards the goal of BWA removal.

In Chapter 2, Table 10 outlined a list of regulations, policies and supporting documents from the federal, provincial/territorial and local level, and the information they provided. It is useful to consider the types of information that would ideally be provided at each level of government. Supporting documents and guidelines from the federal level should encompass information on the three different types of public notifications and clearly define roles and responsibilities of governing and managerial bodies involved with BWAs, as this would promote consistency in terminology and governance across the country. At present, this is not the case. Some guidance in approaches related to the decision-making process for issuing and rescinding BWAs, including steps to take and criteria to consider, can also be provided federally - as it currently is.

All such information should be further built upon and refined in guidance or regulatory documents developed at the provincial/territorial level, since this is the jurisdiction with primary regulatory authority. Specifically, provincial/territorial governments should clearly specify: 1) what the responsibilities of water suppliers and local authorities are; 2) what types of public notification are issuable in the province/territory; 3) what each notification means in definition and practice; 4) when each may be issued (with suggested criteria to consider and steps to take); and 5) when each may be rescinded (with suggested removal criteria to consider and steps to take). The goal should be to provide as much support to decision-makers, in the form of adaptive procedures (incorporating checklists, step-wise actions to take or flowcharts), research, regulations, guidance documents and policies to allow them to structure their decisions across roughly similar yet uniquely different situations. The provincial/territorial government bodies should develop consistent and standardized protocols for the communicating of the public notice to the public, with respect to the type of water system in question and with consideration of the type of public affected.

Other attributes specific to the health authority should be included in documentation produced at the health authority level. Emergency response plans and supporting documentation 
at the water system level should also encompass the same elements discussed above and illustrated in Table 10, but with specifications unique to the water system it is intended for and in laymen's language.

Public notifications should be communicated via methods determined to capture everyone affected and updates on the progress or current status with the public notice should be provided regularly by the health authority (online) and via the water supplier. Each health authority should update their respective web pages on public notifications frequently and should work to provide consistent information and level of detail. Each health authority should also provide links to web pages with further information on what each public notification is, what it means, why it is issued, what the public needs to do to protect their health during the time the notification is in place, and who to contact if more information is needed.

Training is not mandatory for small water system suppliers in BC. This is a discomforting thought, especially given the importance of the role water suppliers have in managing drinking water supplies and protecting public health. All water suppliers should be trained, with the type and level of training dependent on size of water system. This should be well classified in provincial/territorial regulations. Every water system should have an emergency response system in place that details what actions water suppliers need to take as soon as they detect some risk to the drinking water supply. This is stated in the DWPR, but not strictly exercised by all systems. Decisions to issue public notifications, by health authority officials, should be made after considering various applicable criteria and factors (as recommended in guidance documents), any available evidence of risk, and in consultation with the water supplier and other health authority officials, like the MHO, as deemed appropriate. Communication and follow-up between the health authority official and water supplier should be maintained regularly throughout the duration of the public notice. Removal criteria for the public notice should be decided upon by both parties at the time it is decided to issue the notice, and practical strategies and steps should be developed, along with benchmarks and deadlines, to ensure the notice is moving towards removal. 


\subsection{Potential Applications \& Knowledge Translation}

The following is a list of some practical solutions and recommendations for improvement, developed to address some main concerns that health authority officials and water suppliers revealed in their interviews:

1) The decision to issue BWAs is a subjective process and highly variable depending on the decision-maker and the water system under question. Some decision-makers will issue as precaution and others only as last resort. There needs to be some degree of consistency within health regions as well as across BC. This may be possible with a $\mathrm{BC}$-wide toolkit (described below), with materials to assist in decision-making and the overall management of BWAs.

2) Not all water suppliers in BC are trained or fully competent in running a water system, particularly those operating small water systems. The regional pooling of water operators for collective training sessions or workshops has been successfully attempted in the VCHA and could be implemented in other health authorities as well. For more remote water systems, online training and discussions could be utilised.

3) On a similar note, an arrangement that allows for pooled groups of water operators to cover for each other in times of unavailability, or act as support systems, may allow for better maintenance and management of water systems.

4) Changes to the governance of small water systems, where many small water systems are consolidated into one larger regional organisation, may allow for easier sharing of resources (such as staff) as well as improve the overall management of water systems. This governance structure was suggested by a number of health authority officials. Jurisdictions in England and parts of Australia currently use this method of organising drinking water services (60). 
5) Despite water suppliers being deemed primarily responsible for ensuring safe drinking water for their consumers, not all water suppliers are well aware of the risks to drinking water that may result from poorly managed water systems. Education directed to water suppliers for this purpose may be implemented. However, such programs would also need to incorporate initiatives that target the beliefs and attitudes of water suppliers who do not believe in the presence of risk despite being aware of it.

6) There is confusion about the different types of public notifications and when each should be issued. Supporting educational material targeting health authority officials and water suppliers separately, may be helpful. Such information should clearly indicate what the different public notifications are and when they should be issued. All BC health authorities should move towards using the terminology of the Drinking Water Officer's Guide.

7) Not all members of the public under a BWA are receiving the same amount and detail of information. Supporting materials, such as a checklist or pre-made templates, for all BC water systems should be developed for consistency of wording and risk communication information. Each BC health authority's website should aim to provide the same amount and detail of information.

8) Criteria for the removal of BWAs should be developed, in consultation with the water supplier, at the time the BWA is issued. In the case of longstanding BWAs that have been in place for multiple years, concrete criteria should be set indicating when the BWA can be lifted. Ideally, such criteria should be developed after a thorough risk assessment of the water system, so that all possible sources of risk are identified. Some health authority officials mentioned managing BWAs via a staged process. A multi-staged schedule should be created that indicates what specific actions, milestones or criteria should be met and practical deadlines set. This would allow the BWA to be downgraded in stages until it is removed.

9) For certain water systems on long-term BWA, specially community water systems where point-of-use/point-of-entry devices are being introduced, the term "boil water advisory" becomes an inappropriate designation as the community members are not 
boiling the water, but are complying with the BWA differently. This concern was brought up by a few water suppliers. One approach to this could be a new designation rather than "boil water advisory," such as "under household treatment" or "transitioning to decentralised treatment". Implementing this, however, would create the potential for adding more confusion with public notification terminology, and would also downgrade the perception of risk that still exists in households that have not installed a point-of-use/point-of-entry device or those who have installed such devices but have not yet demonstrated satisfactory water quality via water sampling. It is therefore recommended that all health authority websites include, for each BWA listed online, information on: a) why the BWA was issued (the reason); b) what specific actions will determine when the BWA can be lifted (the criteria for removal); and c) up-to-date information regarding the current status of the BWA (updated as soon as new information is available or progress is made).

For example, a community water system moving towards decentralised treatment could have such information provided online:

The reason for the $B W A$ : there is no water treatment or disinfection in place at the water system. [standardised wording] Removal criteria: the BWA will be removed upon the installation of pointof-use/point-of-entry devices in all households and the demonstration of each device's efficacy by water samples that show the water meets the drinking water standards. [standardised wording]

Status as of date/month/year: 14 of the 22 houses have had a device installed. 2 houses are in the process of installing a device.

Such information, if publically available, would allow for a more accurate and complete depiction of the status of the BWA and the water system and may alleviate some of the concerns water suppliers have with the negative perception of BWAs labelling the water system as "bad", noncompliant or mismanaged. Having such information made public may also encourage health authority officials and water suppliers to maintain progress in working towards the BWA's removal, as the process would become more transparent to the public. 


\subsubsection{UBC Program on Water Governance: Water security project toolkit}

This thesis study was supported by the UBC Program on Water Governance and the Canadian Water Network funded project on water security ${ }^{4}$. As a contribution to this project's deliverables, a toolkit will be developed for a Water Security Manual, based on the findings from the thesis research. The goal of this toolkit will be to support and guide water suppliers and health authority officials across BC in the consistent management of BWAs, including the issuing, communication and removal of BWAs. This toolkit is proposed to include the following components:

1) Educational material that defines and describes the 3 main types of public notifications in $\mathrm{BC}$, and in what circumstances each should be issued

2) Detailed steps to take once a possible BWA trigger is identified, including a risk evaluation checklist with criteria to consider when deciding to issue a BWA

3) Steps to take to verify that the BWA has been communicated to the public

4) Supporting material for health authority officials to provide to water suppliers (such as templates and educational information)

5) Material to assist in the development of a timeline to lift the BWA

6) An action plan for health authority officials to implement for water systems that have been on a BWA for more than 1 year for the purposes of developing a timeline and making progress towards the BWA's removal.

The details of this toolkit are in the process of being developed. The drafted toolkit will be offered for review, suggestions and input from health authority officials and water suppliers, and feedback incorporated into the final product prior to release. The completed toolkit will be available online.

\footnotetext{
${ }^{4}$ http://www.watergovernance.ca/projects/water-security/
} 


\subsubsection{Knowledge translation}

Through conference presentations - at local, national and international conferences, such as those held by the Canadian Public Health Association and the American Public Health Association, the findings of the thesis will be shared with other public health professionals and researchers in the field. Results will be specifically shared with EHOs at meetings held by the Canadian Institute of Public Health Inspectors. It is also anticipated that this thesis and its relevant findings will be published in peer-reviewed journals and select association publications, like the Watermark magazine, which is issued to all BC Water and Waste Association members. Participants who indicated an interest in the study's findings will be sent a short report, summarizing this information.

\subsection{Direction of Future Research}

This thesis study reveals problem areas with respect to BWAs and provides impetus for further research. It would be of value to investigate the decision-making process behind issuing BWAs in a larger sample and across all BC health authorities. This would allow testing to determine if health authorities are managing BWAs differently, or whether certain health authorities have developed better strategies than others. It would also allow stratification by size of system, and thus inclusion of more medium and large systems. A longitudinal study that prospectively follows BWAs - from time of issue, including how they are communicated to the public, consequences of the issue thereafter, and public compliance would provide current and complete information. Information from such studies would help provide empirical evidence for the best means by which decisions to issue BWAs should be made. Further studies that look at public compliance and specifically message fatigue on long-standing BWAs or those that frequently issue BWAs, would also be of value, as most studies thus far have focused on shortterm, temporary BWAs. As many water systems had long-standing BWAs in place in lieu of more permanent upgrades to the water system's infrastructure, it may also be of value to collect information on what specific upgrades or actions are effective in improving water systems. Such data and any lessons learned could be used to guide future actions and decisions regarding water system upgrades that work. 


\section{References}

1. Eggertson L. Investigative report: 1766 boil-water advisories now in place across Canada. CMAJ. 2008 May 6; 178 (10):1261-3.

2. Province of British Columbia, Ministry of Health Services. Water advisories in British Columbia - November 2006, Fact Sheet. Health Protection, Population, Health and Wellness; 2006.

3. Office of the Ombudsman. Fit to drink: Challenges in providing safe drinking water in British Columbia. 2008. Report No.: Special Report no. 32 to the Legislative Assembly of British Columbia.

4. Boil water notices/Water quality advisories. [Internet]. Retrieved: February 20, 2011. Available from: http://www.health.gov.bc.ca/protect/dwadvisories.html.

5. Protecting our drinking water [Internet]. Retrieved: February 14, 2011. Available from: http://livingwatersmart.ca/preparation/drinking_water.html.

6. Province of British Columbia. Office of the Provincial Health Officer, Ministry of Health Services. Progress on the action plan for safe drinking water in British Columbia. 2007.

7. Boettger B. In: D.W.P.A. Section 14: Public notice of threats to drinking water. June 15, 2007; [Presentation] British Columbia. 2007.

8. Drinking Water Leadership Council, Province of British Columbia, Ministry of Health Services. Drinking Water Officer's Guide. 2007.

9. Boil water advisories and boil water orders [Internet]. Retrieved: December 3, 2010. Available from: http://www.hc-sc.gc.ca/ewh-semt/pubs/water-eau/boil-ebullitioneng.php.

10. Province of British Columbia. Ministry of Health Services. Turbidity and microbial risk in drinking water. 2008.

11. World Health Organization. Guidelines for drinking water quality. Geneva: WHO; 2004.

12. Maal-Bared R, Bartlett KH, Bowie WR. Dealing with waterborne disease in Canada: Challenges in the delivery of safe drinking water. Rev Environ Health. 2008 Apr-Jun; 23(2):119-33.

13. Davies JM, Mazumder A. Health and environmental policy issues in Canada: The role of watershed management in sustaining clean drinking water quality at surface sources. J Environ Manage. 2003 Jul; 68(3):273-86. 
14. Jones AQ, Majowicz SE, Edge VL, Thomas MK, MacDougall L, Fyfe M, et al. Drinking water consumption patterns in British Columbia: An investigation of associations with demographic factors and acute gastrointestinal illness. Sci Total Environ. 2007 Dec 15; 388(1-3):54-65.

15. Lee M. Risk assessment of drinking water supply failures in Canada [dissertation]. Canada: University of Guelph (Canada); 2007.

16. Hrudey SE, Hrudey EJ, Pollard SJ. Risk management for assuring safe drinking water. Environ Int. 2006 Dec; 32(8):948-57.

17. Health Canada. Public health initiatives related to drinking water quality in Canada by the Safe Drinking Water Subgroup of the Public Health Working Group and Federal/Provincial/Territorial Advisory Committee on Public Health; 2003.

18. O'Donnell M, Platt C, Aston R. Effect of a boil water notice on behaviour in the management of a water contamination incident. Commun Dis Public Health. 2000 Mar; 3(1):56-9.

19. Canadian Drinking Water Guidelines [Internet]. 2010 [updated 2010-12-21]. Retrieved: March 9, 2011. Available from: http://www.hc-sc.gc.ca/ewh-semt/water-eau/drinkpotab/guide/index-eng.php.

20. Province of British Columbia. Provincial Health Officer. A report on the health of British Columbians. Provincial Health Officer's annual report 2000. Drinking water quality in British Columbia: The public health perspective. Victoria, B.C: Ministry of Health Planning; 2001.

21. Drinking Water Protection Act, 2001

22. Harding AK, Anadu EC. Consumer response to public notification. J Am Water Works Assoc. 2000; 92(8):32-41.

23. Health Canada (Federal-Provincial-Territorial Committee on Drinking Water, FederalProvincial-Territorial Committee on Health and the Environment). Guidance For Issuing And Rescinding Boil Water Advisories. Ottawa, Ontario: Water, Air and Climate Change Bureau, Healthy Environments and Consumer Safety Branch, Health Canada; 2009.

24. Clarkson L. In: Boil water advisories: An effective public health tool. June 15, 2007; British Columbia. [Presentation]. 2007.

25. Rundblad G, Knapton O, Hunter PR. Communication, perception and behaviour during a natural disaster involving a 'do not drink' and a subsequent 'boil water' notice: A postal questionnaire study. BMC Public Health. 2010 Oct 25; 10:641. 
26. Karagiannis I, Schimmer B, de Roda Husman AM. Compliance with boil water advice following a water contamination incident in the Netherlands in 2007. Euro Surveill. 2009 Mar 26; 14(12):19156.

27. Willocks LJ, Sufi F, Wall R, Seng C, Swan AV. Compliance with advice to boil drinking water during an outbreak of cryptosporidiosis. Outbreak Investigation Team. Commun Dis Public Health. 2000 Jun; 3(2):137-8.

28. Schell Word CJ. Separate worlds: One small town's compliance problems with water regulations [dissertation]. United States -- Colorado: University of Denver; 1998.

29. Angulo FJ, Tippen S, Sharp DJ, Payne BJ, Collier C, Hill JE, et al. A community waterborne outbreak of salmonellosis and the effectiveness of a boil water order. Am J Public Health. 1997 Apr; 87(4):580-4.

30. Anadu EC. Factors affecting risk perception about drinking water and response to public notification [dissertation]. United States -- Oregon: Oregon State University; 1997.

31. Lacroix BJ. Impact of a boil water advisory on in-home water and food handling practices: A qualitative study [dissertation]. Canada: University of Guelph (Canada); 2007.

32. Wagner MM, Wallstrom GL, Onisko A. Issue a boil-water advisory or wait for definitive information? A decision analysis. AMIA Annu Symp Proc. 2005:774-8.

33. Wallis PM, Matson D, Jones M, Jamieson J. Application of monitoring data for Giardia and Cryptosporidium to boil water advisories. Risk Anal. 2001 Dec; 21(6):1077-85.

34. Province of British Columbia. Office of the Provincial Health Officer, Ministry of Health Services. Progress on the action plan for safe drinking water in British Columbia. 2008.

35. Anon. Canadian town responsible for deaths due to polluted water. Engineering NewsRecord. 1930; 104(2):70.

36. Mayon-White RT, Frankenberg RA. "Boil the water". Lancet. 1989 Jul 22; 2(8656):216.

37. Harrison SL, Nelder R, Hayek L, Mackenzie IF, Casemore DP, Dance D. Managing a large outbreak of cryptosporidiosis: How to investigate and when to decide to lift a 'boil water' notice. Commun Dis Public Health. 2002 Sep; 5(3):230-9.

38. Weber Jr. WJ, Dunahee NK. Boil-water orders: Beneficial or hazardous? J Am Water Works Assoc. 2003; 95(10):40-2.

39. Eggertson L. Despite federal promises, first nations' water problems persist. CMAJ. 2008 Apr 8; 178(8):985. 
40. Rundblad G. The semantics and pragmatics of water notices and the impact on public health. J Water Health. 2008; 6 Suppl 1:77-86.

41. Robertson L, Gjerde B, Hansen EF, Stachurska-Hagen T. A water contamination incident in Oslo, Norway during October 2007; a basis for discussion of boil-water notices and the potential for post-treatment contamination of drinking water supplies. J Water Health. 2009 Mar; 7(1):55-66.

42. Zumpano TAT. Small water systems in British Columbia: Possible obstacles and challenges in management [dissertation]. Canada: Royal Roads University (Canada); 2008.

43. Losito D. Report from the field: Vancouver's boil advisory. Disaster Management Canada. 2006; 1(1):19.

44. Isfeld H. Boil water advisory mapping project: An exploration and review of available data. Winnipeg, Manitoba: 2009. Report No.: Project \#193.

45. Drinking water quality. Drinking water health authority contacts [Internet]. Retrieved: July 15, 2010. Available from: http://www.health.gov.bc.ca/protect/dw_ha_contacts.html

46. Viera AJ, Garrett JM. Understanding interobserver agreement: The kappa statistic. Fam Med. 2005 May; 37(5):360-3.

47. Hrudey SE, Hrudey EJ. A nose for trouble--the role of off-flavours in assuring safe drinking water. Water Sci Technol. 2007; 55(5):239-47.

48. Drinking Water Protection Regulation, 2001.

49. Fraser Health Authority. Environmental Health Services, Health Protection. Guideline: Issuing and Rescinding A Drinking Water Public Notice. 2007.

50. Public Health Protection Management Team, Northern Health Authority. Guideline For Boil Water Notice Decision Process. 2009.

51. Zibin S, Sigalet E, Interior Health Authority. Issue paper: Drinking water public notification policy and guideline recommendation. 2005.

52. Interior Health Authority. In: Interior health: Turbidity Education and Notification Campaign. November 16, 2006; British Columbia. 2006.

53. Turbidity education $\&$ notification campaign ...offering more protection for those at risk [Internet]. Retrieved: February 20, 2011. Available from: http://www.interiorhealth.ca/health-and-safety.aspx?id=526. 
54. Clancy JL. Sydney's 1998 water quality crisis. J Am Water Works Assoc. 2000; 92(3):55-66.

55. Cox P, Fisher I, Kastl G, Jegatheesan V, Warnecke M, Angles M, et al. Sydney 1998lessons from a drinking water crisis. J Am Water Works Assoc. 2003; 95(5):147-61.

56. Haider W, Rasid H. Eliciting public preferences for municipal water supply options. Environmental Impact Assessment Review. 2002 8; 22(4):337-60.

57. Jones AQ. The use and perception of drinking water in a Canadian community [dissertation]. Canada: University of Guelph (Canada); 2006.

58. Nsiah-Kumi PA. Communicating effectively with vulnerable populations during water contamination events. J Water Health. 2008; 6 Suppl 1:63-75.

59. Frewer L. The public and effective risk communication. Toxicol Lett. 2004 Apr 1; 149(13):391-7.

60. Hrudey S. Safe drinking water policy for Canada - turning hindsight into foresight. Commentary. The Water Series. C.D Howe Institute; February 2011. Report No.: No. 323. 


\title{
Appendix A: Letters of Introduction to the Study
}

\author{
THE U N I ERS I T Y OF B R T I S H C O L U M I A
}

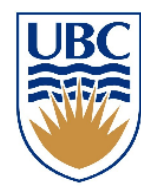

\author{
School of Population and Public Health \\ Mather Building, 5804 Fairview Avenue \\ Vancouver, BC Canada V6T 1Z3 \\ www.spph.ubc.ca \\ phone: \\ email:
}

[date]

[name]

[address]

[city, postal code]

Dear Mr/Ms. [Name of Heath Authority Official],

\section{Re: Boil Water Advisories in British Columbia Study}

I am writing to ask for your participation in a study about boil water advisories in British Columbia. I am trying to understand the decision-making process by which health authority officials and water suppliers issue boil water advisories, how advisories are communicated to the public, how compliance is ensured and what problems or circumstances are hindering the timely removal of long-standing advisories. I hope the study results will improve how boil water advisories are managed in British Columbia by illuminating some of the challenges faced by those involved in water governance.

This study is funded by the Canadian Water Network and is part of the University of British Columbia Program on Water Governance. I am conducting this study as part of my Master of Science thesis at the University of British Columbia.

How were you selected for this study?

Currently ongoing boil water advisories were randomly selected and you were identified as the health authority official responsible for one of the selected advisories. Selected health authority officials from all over British Columbia will take part in this study as well as water suppliers and select members of the public.

What will you need to do to participate?

To participate, you will need to take part in an interview where I will ask you about how the decision to issue the boil water advisory was made, how the advisory was communicated to the public and what specific actions have been taken since the advisory was issued. You are free to decline to answer any question. The interview should take less than an hour and will be audio-taped. It will be conducted by telephone or in-person at a time that is convenient to you. The attached questionnaire lists the questions you will be asked during the interview.

How will your responses be kept confidential?

To protect your confidentiality, your questionnaire and the audio-tape recording will be coded with an ID number, not your name. Our records of your name, address, and telephone number will not include this 
ID number, and will be stored separately from your questionnaire and audio-tape recording. We will keep a record of the number we assign to you, but will not release that record to anyone else. All paper documents containing confidential information will be stored in locked filing cabinets. Electronic data records will be kept on a password protected computer. Only the co-investigator will have access to information about you. All published reports will include only aggregate data and will not include your name or other identifying information.

Is your participation voluntary and how will your consent to participate be requested?

Your participation in this study is entirely voluntary and you may refuse to participate or withdraw from the study at any time. I will telephone you in the next week or so to ask if you are willing to participate. If you are, an interview appointment will be set up. In some studies, participants must sign a consent form, but in this study, it will be assumed that your consent to participate has been given when you agree to set up an appointment for the interview.

How can you contact us?

If you have any questions or concerns about this study, or if you do not wish to be called, please feel free to contact me at [telephone number], [email address], or via the address on this letterhead.

If you have any concerns about your rights or treatment as a research subject, you may contact the Research Subject Information Line in the UBC Office of Research Services at 6048228598 or if long distance, email to RSIL@ors.ubc.ca.

Yours sincerely,

Renuka Grover, BSc

MSc Candidate, UBC 


\section{THE U N I ER S I T Y O F B R I T I S H C O L U M B I A}

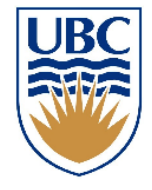

[date]

[name]

[address]

[city, postal code]

Dear Mr/Ms. [Name of Water supplier]
School of Population and Public Health

Mather Building, 5804 Fairview Avenue

Vancouver, BC Canada V6T 1 Z3

www.spph.ubc.ca

phone:

email:

\section{Re: Boil Water Advisories in British Columbia Study}

I am writing to ask for your participation in a study about boil water advisories in British Columbia. I am trying to understand the decision-making process by which health authority officials and water suppliers issue boil water advisories, how advisories are communicated to the public, how compliance is ensured and what problems or circumstances are hindering the timely removal of long-standing advisories. I hope the study results will improve how boil water advisories are managed in British Columbia by illuminating some of the challenges faced by those involved in water governance.

This study is funded by the Canadian Water Network and is part of the University of British Columbia Program on Water Governance. I am conducting this study as part of my Master of Science thesis at the University of British Columbia.

How were you selected for this study?

You were selected because the water system you operate is currently under a boil water advisory. 20 water suppliers from all over British Columbia will take part in this study as well as regional health authority officials and select members of the public.

What will you need to do to participate?

To participate, you will need to take part in an interview, where I will ask you about how the decision to issue the boil water advisory was made, how the advisory was communicated to the public and what specific actions have been taken since the advisory was issued. You are free to decline to answer any question. The interview should take less than an hour and will be audio-taped. It will be conducted by telephone at a time that is convenient to you. The attached questionnaire lists the questions you will be asked during the interview.

How will your responses be kept confidential?

To protect your confidentiality, your questionnaire and the audio-tape recording will be coded with an ID number, not your name. Our records of your name, address, and telephone number will not include this ID number, and will be stored separately from your questionnaire and audio-tape recording. We will keep a record of the number we assign to you, but will not release that record to anyone else. All paper documents containing confidential information will be stored in locked filing cabinets. Electronic data 
records will be kept on a password protected computer. Only the co-investigator will have access to information about you. All published reports will include only aggregate data and will not include your name or other identifying information.

Is your participation voluntary and how will your consent to participate be requested?

Your participation in this study is entirely voluntary and you may refuse to participate or withdraw from the study at any time. I will telephone you in the next week or so to ask if you are willing to participate. If you are, an interview appointment will be set up. In some studies, participants must sign a consent form, but in this study, it will be assumed that your consent to participate has been given when you agree to set up an appointment for the interview.

How can you contact us?

If you have any questions or concerns about this study, or if you do not wish to be called, please feel free to contact me at [phone number],[email address], or via the address on this letterhead.

If you have any concerns about your rights or treatment as a research subject, you may contact the Research Subject Information Line in the UBC Office of Research Services at 6048228598 or if long distance, email to RSIL@ors.ubc.ca.

Yours sincerely,

Renuka Grover, BSc

MSc Candidate, UBC 


\section{Appendix B: Interview Questionnaires with Quantitative Results (shown in red)}

\section{INTERVIEW FORM}

(PARTICIPANT - HEALTH AUTHORITY OFFICIAL)
Interview Number:

Health Authority:

1. Vancouver Coastal

2. Vancouver Island

3. Interior Health

4. Northern Health

5. Fraser Health

Number of interviews conducted by HA:

FHA: $8(0.26)$

IHA: $6(0.19)$

NHA: $9(0.29)$

VCH: $8(0.26)$

VIHA: $0(0)$

TOTAL = $31(1)$

Thank you [name of participant] for agreeing to take part in this study. I really appreciate you taking time from your busy schedule for this. The interview should take about an hour.

In this interview, I will be asking you how the decision to issue the public water notification at [name of water system] came about, how the notification was communicated to the public and what actions have been taken since the issuing of the notification.

Have you had a chance to look over the letter of introduction to the study? Yes $\mid$ No [If no, briefly explain study and participant rights]

YES: 29 (0.94); NO: 2 (0.06)

Do I have your consent to start the interview?

[If yes, proceed; if no, thank him/her for their time and end call]

Yes $\mid$ No YES: 31 (1); NO: 0 (0)

[If name and contact information of water supplier not acquired at initial contact, ask for this information now]

Before we proceed, could you please provide me with the name and contact information of the water supplier at the [name of water system]?

Feel free to stop me and ask questions at any time during the interview. If there is a question that you feel uncomfortable answering, you are welcome to let me know that you don't want to answer it.

Data is based on $31 \mathrm{BWAs}$ at 31 different water systems

18 (0.58) were long-term advisories (in place for at least 6 mo) 13 (0.42) were short-term advisories (no more than 3 mo old)

Means of Interview:

$2 / 31(0.06)=$ in-person

29/31 $(0.94)=$ telephone

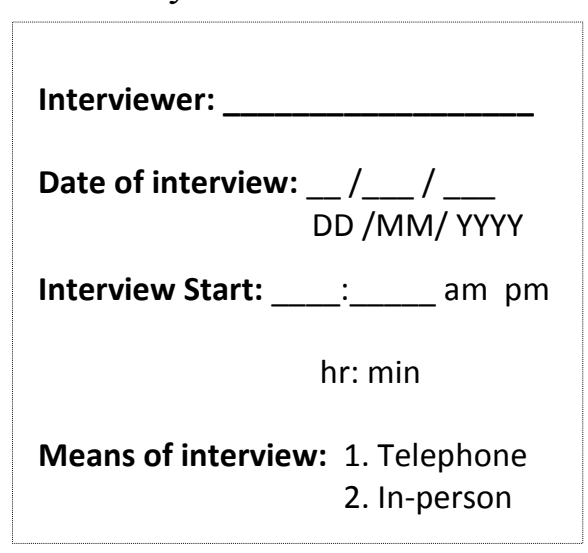


I'd like to start by asking you some general information about yourself in your present role with the [name of health authority].

1. [Record gender]
i. Male
24/31 (0.77)
ii. Female
$7 / 31(0.23)$

Note: 24 different individuals participated. 7 were interviewed more than once but for different BWAs (6 were interviewed on 2 BWAs and 1 on 3 BWAs)

2. What is your current job title at the [name of health authority]?

3. How long have you been working in this position?

Mean: 9.6 years [min $0.17, \max 22]$

4. How many years of experience do you have with drinking water or drinking water programs?

Mean: 13.4 years [min $0.75, \max 36]$

4.1 How many years have you been doing nothing but drinking water?

Mean: 1.7 years [min $0, \max 9]$

5. What are your main responsibilities as related to public information on drinking water quality?

Now I will ask you questions about health threats to drinking water quality. There seems to be differences in the use of terminology about water notification, so I'd like to ask you how you define some terms.

6. Firstly, what classes of public notifications about drinking water does your health authority have? [let interviewee list out public notification classes, read out list; only check off those mentioned by interviewee as stated below]

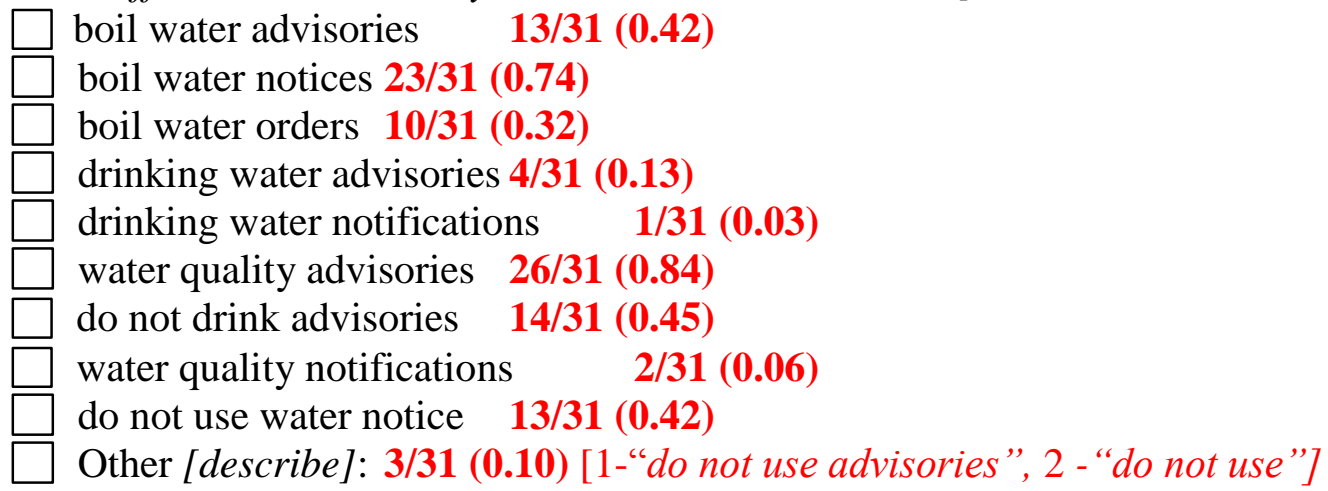


7. For each class of public notification that the interviewee mentioned above, ask]:

a) Under what circumstance are [class of public notification] issued?

b) What, in general, are reasons for issuing [class of public notification]?

c) What is the purpose of [class of public notification]?

8. Are [boil water advisories or the term interviewee uses] issued because there is a risk to health or because of poor water quality?

Risk to health: $\quad 22 / 31(0.71)$

Poor water quality: $\quad \mathbf{1 / 3 1}(\mathbf{0 . 0 3})$

Both: $\quad 8 / 31(0.26)$

9. Do you follow written guidelines on when to issue [boil water advisories]?

i. Yes [If yes, request a copy] 25/31(0.81)

ii. No $4 / 31(0.13)$

iii. Don't know 2/31 (0.06)

10. Is a template or checklist available that lists what information to include in a [boil water advisory] when notifying the public?

i. Yes [if yes, request copy] $\quad 23 / 31(0.74)$

ii. No $8 / 31(0.26)$

iii. Don't know $0(\mathbf{0})$

11. Do you follow written guidelines on when to rescind [boil water advisories]?
i. Yes [if yes, request copy]
$19 / 31(0.61)$
ii. No
9/31 (0.29)
iii. Don't know 3/31 (0.10)

THE WATER SYSTEM UNDER ADVISORY - 1

Now I will ask you questions about the [name of water system].

12. What training and/or certifications does [name of the water supplier] have?

Water supplier has training/certification(s): $10 / 31(0.32)$

Water supplier does not have any training/certification: 19/31 (0.61)

Don't know: 2/31 (0.06)

13. Can you tell me about the organizational structure of the [name of water system]? Who reports to who?

14. From your experience in working with the [name of water system], when there have concerns about drinking water quality or safety at this system, has the water supplier issued a public notification directly or has he contacted the health authority first?

Only HA initiates the process: $18 / 31(0.58)$

Only WS initiates the process: $6 / 31(0.19)$

In the past, both have: $\quad 1 / 31(0.03)$ 
15. How old is the [name of water system]? [if clarification required, this question asks age of water system - ie when started using system to deliver drinking water]

16. How many active connections does this system have?

2-14 connections: $23 / 31(0.74)$

15-300 connections: $8 / 31(0.26)$

301+ connections: $0 / 31(0)$

17. Approximately how many people receive water from this system?

1-10 persons: 9/31 (0.29)

11-50 persons: $10 / 31(0.32)$

51-100 persons: 5/31 (0.16)

101-500 persons: $6 / 31(0.19)$

Population info not available: $1 / 31(0.03)$

Water system runs seasonally only: $8 / 31(0.26)$

Water system runs all year round: 16/31 (0.52)

Don't know: 7/31 (0.22)

18. What is the source of the water supplied by this system?

Surface water source: $11 / 31(0.35)$

Groundwater source: $18 / 31(0.58)$

Other: 2/31 (0.06)

Shallow/dug well (depth less than $15 \mathrm{~m} / 50 \mathrm{ft}): \quad 5 / 31(0.16)$

Deep/drilled well (depth more than $15 \mathrm{~m} / 50 \mathrm{ft}): \quad 14 / 31(0.45)$

Well of unknown depth: $1 / 31(0.03)$

Surface source: $\quad 11 / 31(0.35)$

Other: 1/31 (0.03 [cistern]

19. Are there any barriers or safeguards to this water system in place at the source of water?

i. Yes [If yes, what?] 9/31(0.29)

ii. No 22/31 (0.71)

20. Are there any water treatments in place [at system or POE/POU]?

i. Yes [If yes, what?] 14/31 (0.45)

ii. No $17 / 31(0.55)$

Of the 14 water systems with WT in place:

Have water treatment at 'system': 10/14 (0.71)

Have water treatment at point-of-entry/point-of-use: $4 / 14(0.29)$

Disinfection/treatment in use:

Chlorination: 9/14 (0.64) 
Filtration: 9/14 (0.64)

Ultra-violet: $5 / 14(0.36)$

Ozone: 0/14 (0)

21. Are there any barriers or safeguards in place at the distribution system?

i. Yes [If yes, what?] 7/31 (0.23)

ii. No $24 / 31(0.77)$

22. In the last five years, how many [boil water advisories], including the current one, have been issued on this system?

[If more than 1] 22.1 How long ago was the last [boil water advisory] on this system issued?

22.2 When was it lifted?

22.3 Why was it issued?

\section{WATER NOTIFICATION: DECISION TO ISSUE - 1}

The next set of questions will be about the specific [boil water advisory] currently in place at the [name of water system].

Participant was present at the time of issue (and involved in decision-making): 15/31 (0.48) Participant was not present at the time of issue: 16/31 (0.52)

23. On what date was this [boil water advisory] issued? [ $\mathrm{mm} / \mathrm{dd} / \mathrm{yr}$ ]

[Ensure this date corresponds to start date as listed on the online list of boil water advisories, if not, enquire]

24. What was the reason for issuing this [boil water advisory]? [Water standards or permit requirements not met, malfunction, distribution system problem, loss of integrity in distribution system, new small system without treatment in place, etc.]

25. Which of the following criteria or factors were considered in the decision-process for issuing the [boil water advisory] currently on the system? [check off all that apply]

$\square$ water source $18 / 31(\mathbf{0 . 5 8})$

age of the water system $4 / 31(0.13)$

history of the water system $15 / 31(0.48)$

type of water treatment(s) in place $6 / 31(0.19)$

lack of water treatment(s) in place $23 / 31(0.74)$

mechanical failure of treatment system 5/31 (0.16)

reports of standard failure 20/31 (0.65)

public inconvenience $\mathbf{0} / 31(0)$

public complaints about water quality $1 / 31(\mathbf{0 . 0 3})$

potential liability $6 / 31(0.19)$

timing of event $2 / 31(\mathbf{0 . 0 6})$ 
$\square$ no/limited trained water operator $4 / 31(0.13)$

Other [describe]: 8/31 (0.26)

26. Who was involved in the decision-making process? What role did each play?

In $24 / 31,77 \%$ of the cases, more than one person was involved in the DM process

[If multiple members involved] 26.1 Who made the decision to issue the advisory?

26.2 Was the decision made after multiple deliberations between those involved?

i. Yes $9 / 24(\mathbf{0 . 3 8})$

ii. No $14 / 24(0.58)$

iii. Don't know 1/24 (0.04)

26.3 Were any of the members, yourself included, openly opposed to issuing the [boil water advisory]?

i. Yes $0 / 24(0)$

ii. No $22 / 24(0.92)$

iii. Don't know 2/24 (0.08)

27. When was the decision to issue the advisory made?

28. Were there any reasons not to issue the advisory?
i. Yes [if yes, what?] 3/31 (0.10)
ii. No $27 / 31(0.87)$
iii. Don't know 1/31 (0.03)

29. Were there any alternative actions being considered in place of issuing the [boil water advisory], such as issuing some other form of water notification or trucking water in?

i. Yes [if yes, what?] 3/31 (0.10)

ii. No $27 / 31(0.87)$

iii. Don't know 1/31 (0.03)

\section{WATER NOTIFICATION: PUBLIC COMMUNICATION - 1}

30. Who was responsible for communicating the [boil water advisory] at the [name of water system] to the public?

Health authority only: 0/31 (0)

Water supplier only: 30/31 (0.97)

Both: 1/31 (0.03)

31. By what means was this [boil water advisory] communicated to the public? [Check all that apply; read out list] [1 participant did not know by what means and did not answer] $\square$ Press release $1 / 31(0.03)$

Television $\mathbf{0 / 3 1}(\mathbf{0})$

Radio 1/31 (0.03) 
$\square$ Newspaper 1/31 (0.03)

In person, door-to-door 19/31 (0.61)

Distribution of flyers/print notices to households $7 / 31(0.23)$

Posting flyers/print notices in public places $17 / 31(0.55)$

Telephone 8/31 (0.26)

Other [describe]: 5/31 (0.16)

32. How often was the [boil water advisory] message communicated to the public?

33. Do you have a record of the [boil water advisory] as it was communicated to the public?

i. Yes [if yes, request copy] 9/31 (0.29)

ii. No 22/31 (0.71)

iii. Don't know 0/31 (0)

34. What information was provided to the public about the [boil water advisory]?

[Check all that apply; read out list] [2 participants did not have any info about this]

Date [boil water advisory] issued/effective 20/29 (0.69)

Reason why the advisory was issued 22/29 (0.76)

What the associated health risks are 9/29 (0.31)

Who is at risk $7 / 29(0.24)$

What to do to avoid health risk [if checked, answer 34.1] 29/29 (1)

What corrective action is being taken to end the advisory 9/29 (0.31)

Who to contact for more information/ where to get more information 15/29(0.52)

Additional information for susceptible populations, such as infants and the elderly $3 / 29(0.10)$

How long the advisory is anticipated to last $4 / 29(0.14)$

How the public will be notified of advisory removal $4 / 29(0.14)$

Other [describe]: 3/29 (0.10)

[if checked off "What to do to avoid health risk"]

34.1 What specific actions were the public recommended to take during this

[boil water advisory] to protect their health? [Check all that apply; read out list]

[2 participants did not have any info on this and so could not answer this question]

Boiling tap water before drinking 29/29 (1)

Using bottled water in place of tap water $13 / 29(0.44)$

Treating tap water with household bleach before use $3 / 29(0.10)$

Using other household water treatment 2/29 (0.07)

Using boiled, bottled or treated water for washing fruits and vegetables $6 / 29$

$(0.21)$

Using boiled, bottled or treated water for food and beverage preparation 7/29

(0.24)

Using boiled, bottled or treated water for washing dishes $5 / 29(\mathbf{0 . 1 7})$

Using boiled, bottled or treated water for brushing teeth $7 / 29(0.24)$

Using boiled, bottled or treated water for making ice 4/29 (0.14) 
Giving pets boiled, bottled or treated water for consumption $0 / 29$ (0)

Other [describe]: 9/29 (0.31)

35. Were there any public complaints or questions about the clarity of the advisory message?
i. Yes $\mathbf{1 / 3 1}(\mathbf{0 . 0 3})$
ii. No 24/31 (0.77)
iii. Don't know 6/31 (0.19)

36. Were there any public complaints about the timeliness of the receipt of advisory message?

i. Yes $1 / 31(0.03)$

ii. No 26/31 (0.84)

iii. Don't know 4/31 (0.13)

37. Do you think the public complied with the message recommendations?
i. Yes $\mathbf{1 0 / 3 1}(\mathbf{0 . 3 2})$
ii. No $6 / 31(0.19)$
iii. Don't know 15/31 (0.48)

38. Were any efforts taken to ensure public compliance? If so, what?
i. Yes [if yes, what?] 2/31 (0.06)
ii. No 26/31 (0.84)
iii. Don't know 3/31 (0.10)

\section{WATER NOTIFICATION: THE CONSEQUENCES - 1}

Note: 3 of the 31 BWAs had rescinded by the time of the interview

39. Have you been contacted by members of the public regarding this [boil water advisory]?

i. Yes [if yes, what were their main concerns or questions?] 4/31 (0.13)

ii. No 27/31 (0.87)

iii. Don't know 0/31 (0)

40. Are members of the public regularly updated on the current situation with the boil water advisory?

i. Yes [if yes, how and what information is provided?] 14/31 (0.45)

ii. No 11/31 (0.35)

iii. Don't know 6/31 (0.19)

41. What resources are available for the public to access for more information about the current [boil water advisory]? Who is the primary contact person for members of the public to reach for information regarding this current [boil water advisory]?

42. Are there any criteria or benchmarks set in place to help indicate when this [boil water advisory] can be lifted?

i. Yes [if yes, what?] 28/31 (0.90) 

ii. No $3 / 31(0.10)$
iii. Don't know 0/31 (0)

43. Since the issuance of the [boil water advisory] at the [name of water system], what actions have been taken in effort to lead to the removal of the advisory?

44. What action(s) are pending and need to be done before the advisory can be lifted?

45. Who has primary responsibility for ensuring the problem leading to the issuing of the boil water advisory is being addressed?

46. Have you followed-up with [name of the water supplier] since the issuing of the advisory?

i. Yes 31/31 (1)

ii. No $0 / 31(0)$

iii. Don't know 0/31 (0)

[If yes] 46.1 When was the last time you communicated with the water supplier regarding the advisory?

46. 2 What was the outcome of your most recent communication with the water supplier?

47. Who will make the decision on when to lift this [boil water advisory]?

48. Is there an anticipated date by which the advisory will be lifted?

i. Yes [if yes, when?] 6/31 (0.19)

ii. No 24/31 (0.77)

iii. Don't know 1/31 (0.03)

49. Are there any challenges or obstacles that are hindering the removal of this [boil water advisory]?

i. Yes [if yes, what?] 24/31 (0.77)

ii. No 7/31 (0.23)

iii. Don't know 0/31 (0)

\section{OPINIONS \& COMMENTS}

In the final set of questions, I will ask for your comments and opinions on topics related to drinking water management in BC.

50. What are the challenges faced in the decision-making process for issuing and rescinding advisories?

51. Do you have any suggestions as to how to streamline or improve the decision-making process? 
52. What are the challenges with the public notification of drinking water quality?

53. In your opinion, are [boil water advisories] an effective preventative measure in protecting the public from acquiring waterborne illnesses? Why or why not?

54. In your opinion, is there any water treatment process that should be a mandatory in every water system in BC? If so, which ones and why. If not, why not?

55. In your opinion, should water filtration be mandatory in every water system in BC? Why or why not?

56. In your opinion, how much evidence indicating threat to drinking water safety or quality do you think there should be before the decision to issue a [boil water advisory] is made?

57. Do you think public compliance towards [boil water advisories] is of any concern?

58. Do you know about the Walkerton, Ontario outbreak? If yes, in your experience, has anything changed with regards to the management of drinking water and [boil water advisories] since the Walkerton outbreak in 2000 ?

59. Finally, do you have any comments you would like to add, for example, about the [boil water advisory], this interview, this study, or about drinking water in general?

That's it! Thank you so much for participating in this study.

Would you be interested in seeing the results of this study?

[If yes: I will send you a summary of the results once the study has completed].

Thank you again for your time. If you ever have any questions, feel free to call me.

Interview End: am pm 
Thank you [name of participant] for agreeing to take part in this study. I really appreciate you taking time from your busy schedule for this. The interview should take no more than an hour.

In this interview, I will be asking you how the decision to issue the public water notification at [name of water system] came about, how the notification was communicated to the public and what actions have been taken since the issuing of the notification.

Have you had a chance to look over the letter of introduction to the study?

[If no, briefly explain study and participant rights]

Do I have your consent to start the interview?

[If yes, proceed; if no, thank him/her for their time and end call]

Feel free to stop me and ask questions at any time during the interview. If there is a question that you feel uncomfortable answering, you are welcome to let me know that you don't want to answer it.

Data is based on 14 of 31 selected BWAs at 14 different water systems

$8(\mathbf{0 . 5 7})$ were long-term advisories (in place for at least $6 \mathrm{mo}$ )

$\mathbf{6}(\mathbf{0 . 4 3})$ were short-term advisories (no more than 3 mo old)

Means of Interview:

0/14 (0) $=$ in-person

$12 / 14(0.86)=$ telephone

$1 / 14(0.07)=$ email

$1 / 14(0.07=$ mail

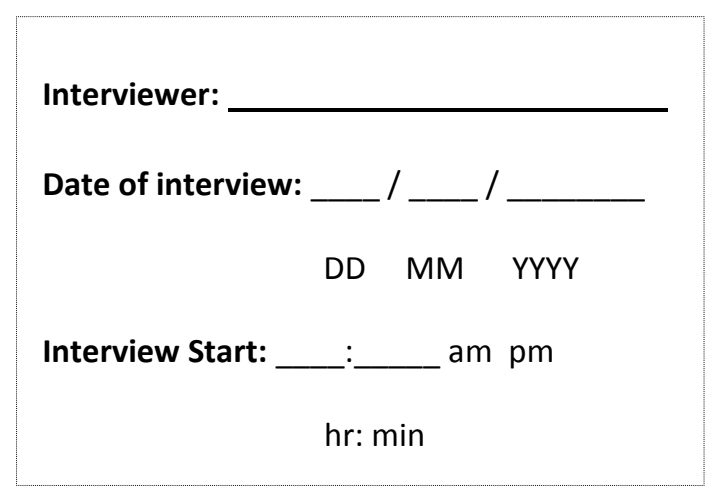


I'd like to start by asking you some general information about yourself in your present role at the [name of water system].

1. [Record gender]
i. Male 9/14 (0.64)
ii. Female $4 / 14(0.29)$
iii. No info $1 / 14(\mathbf{0 . 0 7})$

2. What is your current job title at the [name of water system]?

3. How long have you been working in this position?

Mean: 7.6 years [min: 1 , max: 20]

4. How many years of experience do you have in the field of water management?

Mean: 7.0 years [min: 0, max: 25]

5. What training and/or certifications do you have?

Has training/certification(s): 7/14 (0.50)

Does not have any training/certification: $7 / 14(0.50)$

6. What are your main responsibilities as related to public information of drinking water quality?

7. Can you tell me about the organizational structure of your water system? Who reports to who?

8. In general, when you have concerns about drinking water quality or safety at the water system, do you issue a public notification or do you contact the health authority?

Only HA initiates the process: 8/14 (0.57)

Only WS initiates the process: $2 / 14(0.14)$

In the past, both have: $\quad 2 / 14(0.14)$

Don't know: 2/14 (0.14)

Now I will ask you questions about health threats to drinking water quality. There seems to be differences in the use of terminology about water notification, so I'd like to ask you how you define some terms.

9. Firstly, what classes of public notifications about drinking water in the [name of health authority] are issuable on your water system?? [ let interviewee list out public notification classes, read out list; only check off those mentioned by interviewee as stated below]

$\square$ boil water advisories $\quad 11 / 14(0.79)$

boil water notices $4 / 14(0.29)$

boil water orders $1 / 14(0.07)$ 
$\square$ drinking water advisories 1/14 (0.07)

drinking water notifications $\quad 1 / 14(0.07)$

water quality advisories $3 / 14(0.21)$

do not drink advisories $2 / 14(0.14)$

water quality notifications $\quad 0 / 14(0)$

do not use water notice $1 / 14(0.07)$

Other [describe]: 2/14 (0.14) [1- 'do not drink notice', 1 - 'drinking advisory']

10. [For each class of public notification that the interviewee mentioned above, ask]:

a) Under what circumstance are [class of public notification] issued?

b) What, in general, are reasons for issuing [class of public notification]?

c) What is the purpose of [class of public notification]?

11. Are [boil water advisories or the term interviewee uses] issued because there is a risk to health or because of poor water quality?
Risk to health:
$4 / 14(0.29)$
Poor water quality: $\quad 3 / 14(0.21)$
Both: $\quad 5 / 14(0.35)$
Don't know: $\quad$ 2/14 (0.14)

12. Do you follow written guidelines on when to issue [boil water advisories]?
i. Yes [If yes, request a copy]
$7 / 14(0.50)$
ii. No $6 / 14(\mathbf{0 . 4 3})$
iii. Don't know 1/14 (0.07)

13. Is a template or checklist available that lists what information to include in a [boil water advisory] when notifying the public?

i. Yes [if yes, request copy] 7/14 (0.50)

ii. No $5 / 14(0.36)$

iii. Don't know 1/14 (0.07)

iv. N/A 1/14 (0.07)

14. Do you follow written guidelines on when to rescind [boil water advisories]?

i. Yes [if yes, request copy] 6/14 (0.43)

ii. No 6/14 (0.43)

iii. Don't know 2/14 (0.14)

\section{THE WATER SYSTEM UNDER ADVISORY}

Now I will ask you questions about the [name of water system].

15. What is the name of the health authority official responsible for this water system and with whom you communicate regarding boil water advisories?

16. How old is this water system? [if clarification required, this question asks age of water system - ie when started using system to deliver drinking water] 
17. How many active connections does this system have?

2-14 connections: $10 / 14(0.71)$

15-300 connections: $4 / 14(0.29)$

301+ connections: 0/14 (0)

18. Approximately, how many people receive water from this system?

1-10 persons: $1 / 14(0.07)$

11-50 persons: 6/14 (0.43)

51-100 persons: $2 / 14(0.14)$

101-500 persons: 5/14 (0.36)

Water system runs seasonally only: $4 / 14(0.29)$

Water system runs all year round: $7 / 14(0.50)$

Don't know: $3 / 14$ (0.21)

19. What is the source of the water supplied by this system?

Surface water source: $4 / 14(0.29)$

Ground water source: $9 / 14(0.64)$

Other: 1/14 (0.07) \{mix of surface and ground\}

Shallow/dug well (depth less than 15m/50ft): $\quad 3 / 14(0.21)$

Deep/drilled well (depth more than $15 \mathrm{~m} / 50 \mathrm{ft}): \quad 5 / 14(0.36)$

Well of unknown depth: $1 / 14(0.07)$

Surface source: $\quad 5 / 14(0.36)$

Other: 0/14 (0)

20. Are there any barriers or safeguards to this water system in place at the source of water?

iii. Yes [If yes, what?] 13/14 (0.93)

iv. No $1 / 14(0.07)$

21. Are there any water treatments in place?

i. Yes [If yes, what?] 9/14 (0.64)

ii. No $5 / 14(0.36)$

Of the 9 water systems with WT in place:

Have water treatment at 'system': $8 / 9$ (0.89)

Have water treatment at point-of-entry/point-of-use: $1 / 9(0.11)$

Disinfection/treatment in use:

Chlorination: 5/9 (0.56)

Filtration: $3 / 9(0.33)$

Ultra-violet: $3 / 9(0.33)$

Ozone: 0/9 (0)

No info given: $2 / 9(0.22)$ 
22. Are there any barriers or safeguards in place at the distribution system?
i. Yes [If yes, what?] 7/14 (0.50)
ii. No 6/14 (0.43)
iii. Don't know 1/14 (0.07)

23. In the last five years, how many [boil water advisories], including the current one, have been issued on this system?

[If more than 1] 23.1 When was the last [boil water advisory] on this system issued? 23.2 When was it lifted?

23.3 Why was it issued?

\section{WATER NOTIFICATION: DECISION TO ISSUE}

The next set of questions will be about the specific [boil water advisory] currently in place at the [name of water system].

Participant was present at the time of issue (and involved in decision-making): 6/14 (0.43) Participant was not present at the time of issue: $8 / 14(0.57)$

24. On what date was this boil water advisory issued? [ $\mathrm{mm} / \mathrm{dd} / \mathrm{yr}]$

[Ensure this date corresponds to start date as listed on the online list of BWAs, if not, enquire]

25. What was the reason for issuing this boil water advisory? [Water standards or permit requirements not met, malfunction, distribution system problem, loss of integrity in distribution system, newly discovered small system without treatment in place, etc.]

26. Which of the following criteria or factors were considered in the decision-process for issuing the [boil water advisory] currently on the system? [check off all that apply] [2 participants did not know the decision factors/criteria and didn't answer this question] water source $5 / 14(0.36)$ age of the water system $1 / 14(0.07)$ history of the water system $1 / 14(0.07)$ type of water treatment(s) in place 1/14 (0.07) lack of water treatment(s) in place 5/14 (0.36) mechanical failure of treatment system 2/14 (0.14) reports of standard failure $4 / 14(0.29)$ public inconvenience 1/14 (0.07) public complaints about water quality $0 / 14(0)$ potential liability $2 / 14(0.14)$ timing of event $1 / 14(\mathbf{0 . 0 7})$

Other [describe]: 2/14 (0.14) 
27. Who was involved in the decision-making process? What role did each play?

In $7 / 14,50 \%$ of the cases, more than one person was involved in the DM process

[If multiple members involved] 27.1 Who made the decision to issue the advisory?

27.2 Was the decision made after multiple deliberations between those involved?
i. Yes $4 / 7(0.57)$
ii. No $3 / 7(0.43)$
iii. Don't know $\mathbf{0} / 7$ (0)

27.3 Were any of the members, yourself included, openly opposed to issuing the [boil water advisory]?

i. Yes $0 / 7(\mathbf{0})$

ii. No $7 / 7(1)$

iii. Don't know 0/7 (0)

28. When was the decision to issue the advisory made?

29. Were there any reasons not to issue the advisory?

i. Yes [if yes, what?] 1/14 (0.07)

ii. No $11 / 14(0.79)$

iii. Don't know 2/14 (0.14)

30. Were there any alternative actions being considered in place of issuing the [boil water advisory], such as issuing some other form of water notification or trucking water in?

i. Yes [if yes, what?] 2/14 (0.14)

ii. No $9 / 14(0.64)$

iii. Don't know 3/14 (0.21)

\section{WATER NOTIFICATION: PUBLIC COMMUNICATION}

31. Who was responsible for communicating the [boil water advisory] at the [name of water system] to the public?

Health authority only: 3/14 (0.21)

Water supplier only: 11/14 (0.79)

Both: 0/14 (0)

32. By what means was this [boil water advisory] communicated to the public? [Check all that apply; read out list]

$\square$ Press release $\mathbf{0} / 14(0)$

$\square$ Television $\mathbf{0 / 1 4}(\mathbf{0})$

$\square$ Radio $\quad 0 / 14(0)$

Newspaper 2/14 (0.14)

In person, door-to-door 7/14 (0.50)

$\square$ Distribution of flyers/print notices to households $6 / 14(0.43)$ 
$\square$ Posting flyers/print notices in public places $8 / 14(0.57)$

Telephone $\mathbf{5 / 1 4}(\mathbf{0 . 3 6})$

Other [describe]: $6 / 14(0.43)$

33. How often was the [boil water advisory] message communicated to the public?

34. Do you have a record of the [boil water advisory] as it was communicated to the public?

i. Yes [if yes, request copy] 11/14 (0.79)

ii. No $3 / 14(0.21)$

iii. Don't know 0/14 (0)

35. What information was provided to the public about the [boil water advisory]?

[Check all that apply; read out list]

[1 participant did not know and could not answer this question]

Date [boil water advisory] issued/effective $8 / 13(0.62)$

Reason why the advisory was issued $6 / 13(0.46)$

What the associated health risks are $1 / 13(\mathbf{0 . 0 8})$

Who is at risk $1 / 13(\mathbf{0 . 0 8})$

$\square$ What to do to avoid health risk [if checked, answer 35.1] 13/13 (1)

$\square$ What corrective action is being taken to end the advisory $2 / 13(0.15)$

Who to contact for more information/ where to get more information 4/13 (0.31)

Additional information for susceptible populations, such as infants and the elderly $1 / 13(0.08)$

$\square$ How long the advisory is anticipated to last $2 / 13(0.15)$

How the public will be notified of advisory removal $4 / 13(0.31)$

Other [describe]: 3/13 (0.23)

[if checked off "What to do to avoid health risk"]

What specific actions were the public recommended to take during this [boil water advisory] to protect their health?

35.1 [Check all that apply; read out list]

Boiling tap water before drinking 9/13 (0.64)

Using bottled water in place of tap water $7 / 13(0.54)$

Treating tap water with household bleach before use 2/13 (0.15)

Using other household water treatment 2/13 (0.15)

$\square$ Using boiled, bottled or treated water for washing fruits and vegetables 7/13

$(0.54)$

$\square$ Using boiled, bottled or treated water for food and beverage preparation 7/13 (0.54)

Using boiled, bottled or treated water for washing dishes $5 / 13(0.38)$

$\square$ Using boiled, bottled or treated water for brushing teeth 5/13 (0.38)

$\square$ Using boiled, bottled or treated water for making ice 5/13 (0.38)

$\square$ Giving pets boiled, bottled or treated water for consumption

$1 / 14(0.08)$

$\square$ Other [describe]: 4/13 (0.31) 
36. Were there any public complaints or questions about the clarity of the advisory message?

i. Yes $\mathbf{1} / \mathbf{1 4}(\mathbf{0 . 0 7 )}$

ii. No $13 / 14(0.93)$

iii. Don't know 0/14 (0)

37. Were there any public complaints about the timeliness of the receipt of advisory message?
i. Yes $\mathbf{1} / 14(0.07)$
ii. No $13 / 14(0.93)$
iii. Don't know 0/14 (0)

38. Do you think the public complied with the message recommendations?
i. Yes $9 / 14(0.64)$
ii. No $2 / 14(0.14)$
iii. Don't know 3/14 (0.21)

39. Were any efforts taken to ensure public compliance? If so, what?
i. Yes [if yes, what?] 10/14 (0.71)
ii. No $4 / 14(0.29)$
iii. Don't know 0/14 (0)

\section{BOIL WATER ADVISORY: THE CONSEQUENCES}

40. Have you been contacted by members of the public regarding this [boil water advisory]?
i. Yes [if yes, what were their main concerns or questions?] 3/14 (0.21)
ii. No $11 / 14(0.79)$
iii. Don't know 0/14 (0)

41. Are members of the public regularly updated on the current situation with the boil water advisory?

i. Yes [if yes, how and what information is provided?] 7/14 (0.50)

ii. No $6 / 14(0.43)$

iii. Don't know 1/14 (0.07)

42. What resources are available for the public to access for more information about this current [boil water advisory]? Who is the primary contact person for members of the public to reach for information regarding this current [boil water advisory]?

43. Are there any criteria or benchmarks set in place to help indicate when this [boil water advisory] can be lifted?

i. Yes [if yes, what?] 12/14 (0.86)

ii. No $1 / 14(0.07)$

iii. Don't know 1/14 (0.07) 
44. Since the issuance of the boil water advisory at the [name of water system], what actions have been taken in effort to lead to the removal of the advisory?

45. What action(s) are pending and need to be done before the advisory can be lifted?

46. Who has primary responsibility for ensuring the problem leading to the issuing of the boil water advisory is being addressed?

47. Have you followed-up with [name of the health authority official] since the issuing of the advisory?

i. Yes $14 / 14(1)$

ii. No 0/14 (0)

iii. Don't know 0/14 (0)

[If yes] 47.1 When was the last time you communicated with the health authority regarding the advisory?

47. 2 What was the outcome of your most recent communication with the health authority?

48. Who will make the decision on when to lift this [boil water advisory]?

49. Is there an anticipated date by which the advisory will be lifted?

i. Yes [if yes, when?] $6 / 14(0.43)$

ii. No $7 / 14(0.50)$

iii. Don't know $\mathbf{0 / 1 4}(\mathbf{0})$

iv. No answer $\mathbf{1 / 1 4}(\mathbf{0 . 0 7})$

50. Are there any challenges or obstacles that are hindering the removal of this [boil water advisory]?

i. Yes [if yes, what?] 8/14 (0.57)

ii. No 6/14 (0.43)

iii. Don't know 0/14 (0)

\section{OPINIONS \& COMMENTS}

In the final set of questions, I will ask for your comments and opinions on topics related to drinking water management in $\mathrm{BC}$.

51. What are the challenges faced in the decision-making process for issuing and rescinding advisories?

52. Do you have any suggestions as to how to streamline or improve the decision-making process? 
53. What are the challenges with the public notification of drinking water quality?

54. In your opinion, are boil water advisories an effective preventative measure in protecting the public from acquiring waterborne illnesses? Why or why not?

55. In your opinion, is there any water treatment process that should be a mandatory in every water system in $\mathrm{BC}$ ? If so, which ones and why. If not, why not?

56. In your opinion, should water filtration be mandatory in every water system in BC? Why or why not?

57. In your opinion, how much evidence indicating threat to drinking water safety or quality do you think there should be before the decision to issue a [boil water advisory] is made?

58. Do you think public compliance towards boil water advisories is of any concern?

59. Do you know about the Walkerton, Ontario outbreak? If yes, in your experience, has anything changed with regards to the management of drinking water and boil water advisories since the Walkerton outbreak in 2000?

60. Finally, do you have any comments you would like to add, for example, about the [boil water advisory], this interview, this study, or about drinking water in general?

That's it! Thank you so much for participating in this study.

Would you be interested in seeing the results of this study?

[If yes: I will send you a summary of the results once the study has completed].

Thank you again for your time. If you ever have any questions, feel free to call me.

Interview End: am pm 


\section{Appendix C: Public/Water Consumer Interview Questionnaire with Quantitative}

Results (in red)

* This segment of the study was excluded from the thesis

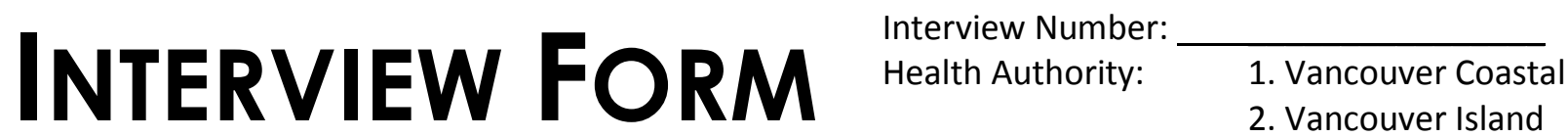 \\ 3. Interior Health \\ (PARTICIPANT - MEMBER OF PUBLIC) \\ 4. Northern Health \\ 5. Fraser Health}

FHA: 3 (0.23); IHA: $3(0.23)$

NHA: 4 (0.31); VCHA: $3(0.23)$

Total: 13

Thank you [name of participant] for agreeing to take part in this study. The interview should take about 30 minutes.

In this interview, I will be asking you about your water supply.

Have you had a chance to look over the letter of introduction to the study? Yes $\mid$ No

YES: 4 (0.31); NO: $9(0.69)$

[If no, briefly explain study and participant rights]

Do I have your consent to start the interview?

[If yes, proceed; if no, thank him/her for their time and end call]

Yes $\mid$ No

YES: 13 (1); NO: 0 (0)

Are there any other questions you'd like me to answer before we begin the interview?

[Give time to answer]

Feel free to stop me and ask questions at any time during the interview. If there is a question that you feel uncomfortable answering, you are welcome to let me know that you don't want to answer it.

Data is based on BWAs at 7 different water systems

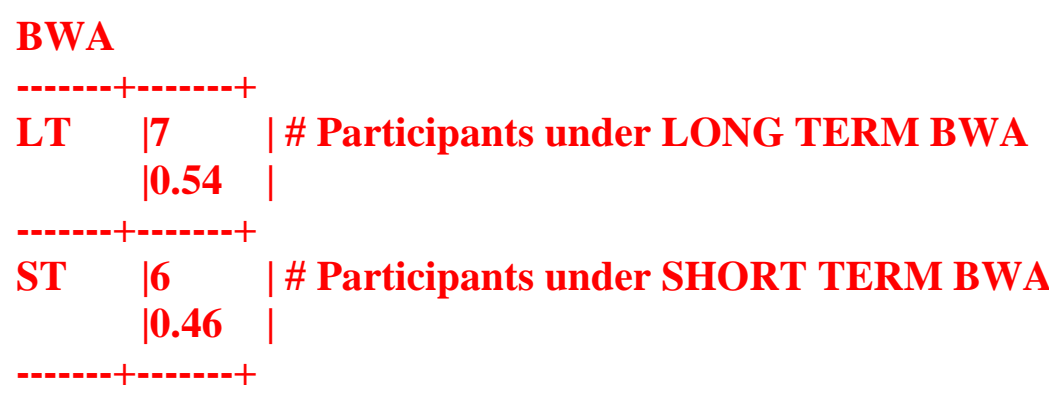

Total 13
Interviewer:
Date of interview:
Interview Start: $\frac{\mathrm{DD}}{\mathrm{hr}: \min } \mathrm{am} \mathrm{pm}$
Means of interview: 1. Telephone

2. In-person 
Means of Interview: TELEPHONE: 13 (1); IN-PERSON: 0 (0)

Participant 'type':

Resident: $10(0.77)$

Staff: $1(0.077)$

Both: 2 (0.15)

\section{AWARENESS OF HEALTH AUTHORITY \& WATER SUPPLIER}

I will start by asking you questions about your health authority and water supplier.

1. The Province of British Columbia is divided into five geographically distinct health authorities that regionally manage and deliver health care services. According to where you live, do you know which health authority you are in?
i. Yes
$6 / 13(0.46)$
ii. No
$7 / 13(0.54)$
iii. Unsure

[if yes] 1.1. Which health authority?

[Did participant indicate correct health authority?

1. Yes / O. No]

Of the 6 who said YES, all 6 correctly identified their health authority

1.2. In the last five years, have you contacted your health authority for any issues having to do with your drinking water?
i. Yes
$0 / 6(0)$
ii. No
$6 / 6(1)$

[if yes] 1.2.1. What was the reason for contact?

1.2.2. What was the outcome of the contact?

1.3. In the last five years, has the health authority contacted you for any issues having to do with your drinking water?
i. Yes

2/6 (0.33)

ii. No $4 / 6(0.67)$

[if yes] 1.3.1. What was the reason for contact?

1.3.2. What was the outcome of the contact?

2. Do you know the name of the water system that supplies your household with water?
i. Yes
$8 / 13(0.61)$
ii. No
$4 / 13(0.31)$
iii. Unsure 1/13(0.08)

[if yes] 2.1. Who is your water supplier?

[Did participant indicate correct water supplier?

1. Yes / 0. No]

Of the 8 who said YES, all 8 correctly identified their water supplier 
2.2. In the last five years, have you contacted your water supplier for any issues having to do with your drinking water?

i. Yes $3 / 9(0.33)$

ii. No $6 / 9(0.67)$

[if yes] 2.2.1. What was the reason for contact?

2.2.2. What was the outcome of the contact?

2.3. In the last five years, has the water supplier ever contacted you for any issues having to do with your drinking water?

i. Yes 6/9 (0.67)

ii. No $3 / 9(0.33)$

[if yes] 2.3.1. What was the reason for contact?

2.3.2. What was the outcome of the contact?

\section{AWARENESS AND UNDERSTANDING OF BWA}

In the next set of questions, I will ask you about the boil water advisory that is being investigated in this study.

3. Prior to being contacted for participation in this study, were you aware of the boil water advisory that is currently on the water system that supplies your home with water?
i. Yes
$13 / 13(1)$
ii. No
$0 / 13(0)$

[if no] 3.1 What would have been the best way for you to be informed of the boil water advisory? [Do not read out list]

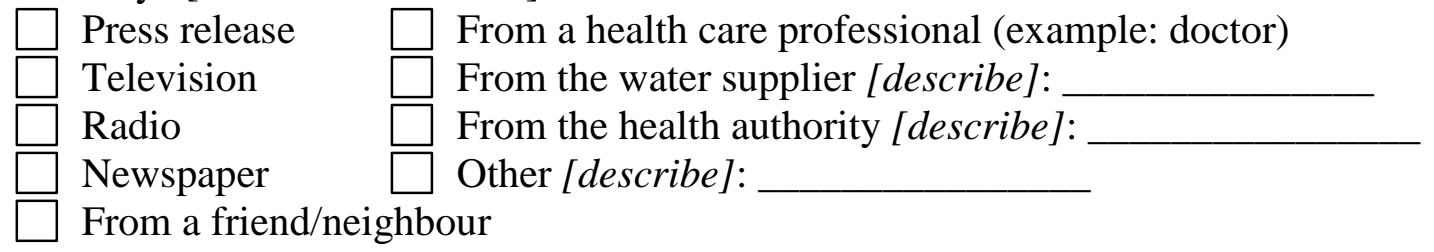

[if no] 3.2. What information would you have wanted to be provided with in the boil water advisory message?

[if no, skip to question 25]

\section{[if yes, proceed with interview]}

4. Can you recall approximately when this boil water advisory was issued?
i. Yes
9/13 (0.69)
ii. No
$4 / 13(0.31)$
[if yes] When? [Date:

5. Do you know why this boil water advisory was issued?
i. Yes
$12 / 13(0.92)$
ii. No
$1 / 13(0.08)$

[if yes] 5.1. What was the reason? 
6. How did you first come to know about the boil water advisory? [Check one; Do not read out list; if interviewee mentions more than 1, check off the one mentioned first here and the others in the next question]

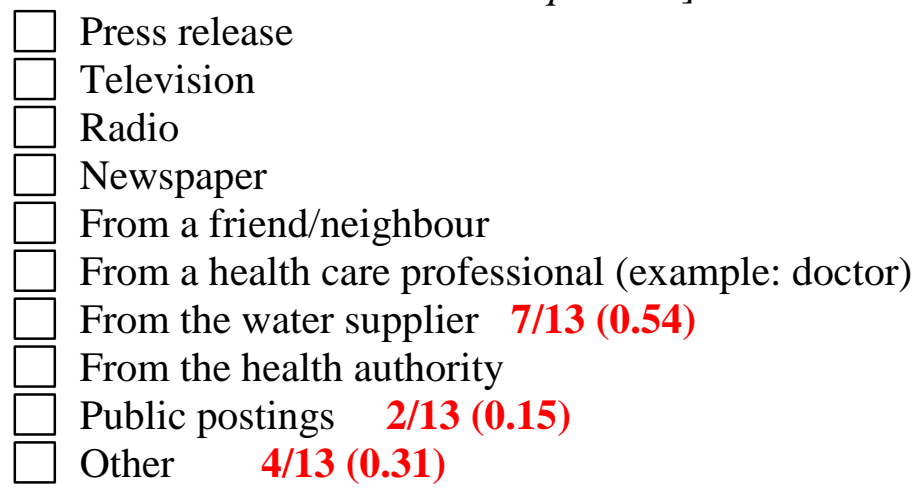

7. By what other means did you hear about the boil water advisory?

[Check all that apply; Read out list excluding the one mentioned in question 6]

\begin{tabular}{|c|c|c|c|}
\hline & Press release & 0/13 (0) & \\
\hline & Television & 0/13 (0) & \\
\hline & Radio & & \\
\hline & Newspaper & $\mathbf{0} / \mathbf{1 3}(\mathbf{0})$ & \\
\hline & Text messag & SMS via cell phone mobility com & $0 / 13(0)$ \\
\hline & From a frien & eighbour $7 / 13(0.54)$ & \\
\hline & From a healt & ire professional (example: doctor) & $\mathbf{0} / \mathbf{1 3}(\mathbf{0})$ \\
\hline & From the wa & supplier $2 / 13(0.15)$ & \\
\hline & From the he: & authority & \\
\hline & Other & (0.61) & \\
\hline
\end{tabular}

8. Would you have preferred to receive the boil water advisory by some other means?
i. Yes $2 / 13(0.15)$
ii. No $11 / 13(0.85)$
[if yes] $\mathbf{8 . 1}$ How?

9. What are your thoughts on the timeliness of the receipt of the boil water advisory? Would you say that you were:

i. informed of the boil water advisory in time. $\quad 10 / 13(0.77)$

ii. not informed of the boil water advisory in time. 2/13 (0.15)

iii. DK $1 / 13(0.08)$

10. What information were you provided in the boil water advisory?

[Check all that apply; read out list]

$\square$ Date [boil water advisory] issued/effective $\quad 12 / 13(0.92)$

Reason why the advisory was issued $\quad 8 / 13(0.61)$

What the associated health risks are $\quad 7 / 13(0.54)$

Who is at risk $\quad 8 / 13(0.61)$

What to do to avoid health risk [if checked, answer 10.1]

$13 / 13(1)$ 
$\square$ What corrective action is being taken by the supplier to end the advisory $6 / 13(0.46)$

Who to contact for more information/ where to get more information 9/13 (0.69)

Additional info for susceptible populations, such as infants/the elderly 2/13 (0.15)

How long the advisory is anticipated to last $\quad 4 / 13(0.31)$

How the public will be notified of advisory removal $2 / 13(0.15)$

Other [describe]:

$0 / 13(0)$

[if checked off "What to do to avoid health risk"]

10.1. What specific actions were you recommended to take during this the boil water advisory to protect the health of yourself and your family?

[Read out list; Check all that apply]

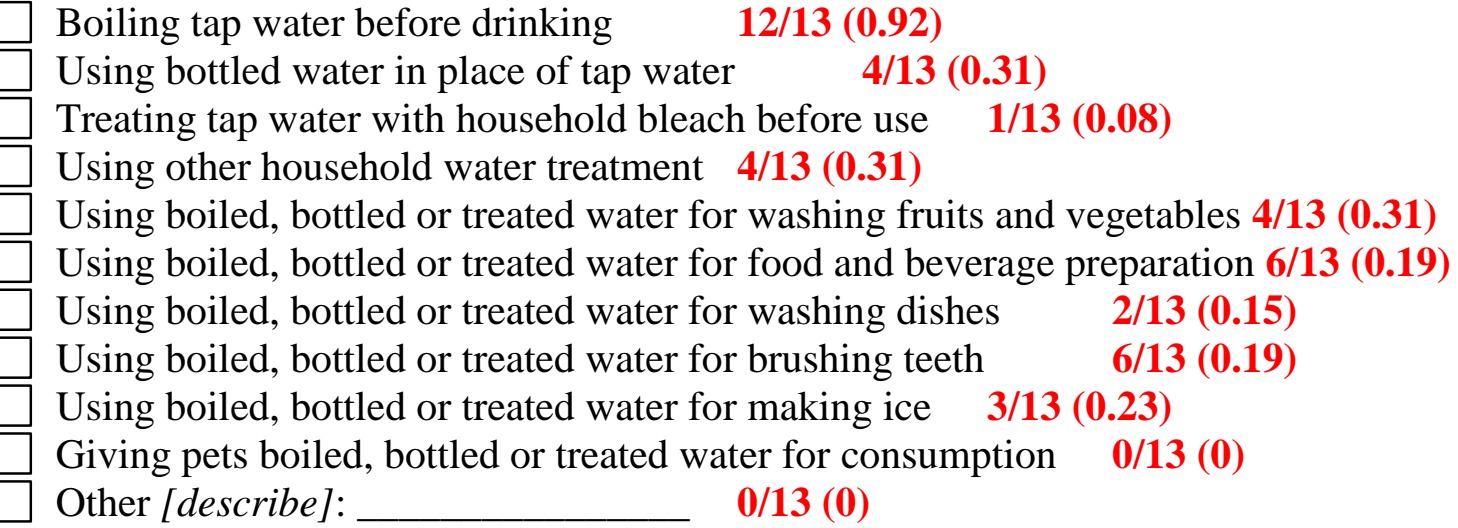

11. Is there any information you were not provided in the boil water advisory that you would have liked to know?
i. Yes
$5 / 13(0.38)$
ii. No
$8 / 13(0.61)$
[if yes] 11.1. What?

12. Are you receiving updates on the boil water advisory from your health authority or water supplier directly, for instance on what action is currently being taken to correct the problem or by when the advisory should be removed?
i. Yes
$7 / 13(0.46)$
ii. No
$6 / 13(0.54)$

[if yes] 12.1. When were you last informed or updated?

[if yes] 12.2. What information were you provided?

13. Since boil water advisory was issued, have you received updates on the advisory from the media, for instance, through flyers, TV or radio announcements?
i. Yes
0/13 (0)
ii. No
13/13 (1)

[if yes] 13.1. When were you last informed or updated?

[if yes] 13.2. What information were you provided? 
Now I will ask you questions on what specific measures you have been taking during the boil water advisory.

Since the boil water advisory was issued on your water system, have you been:

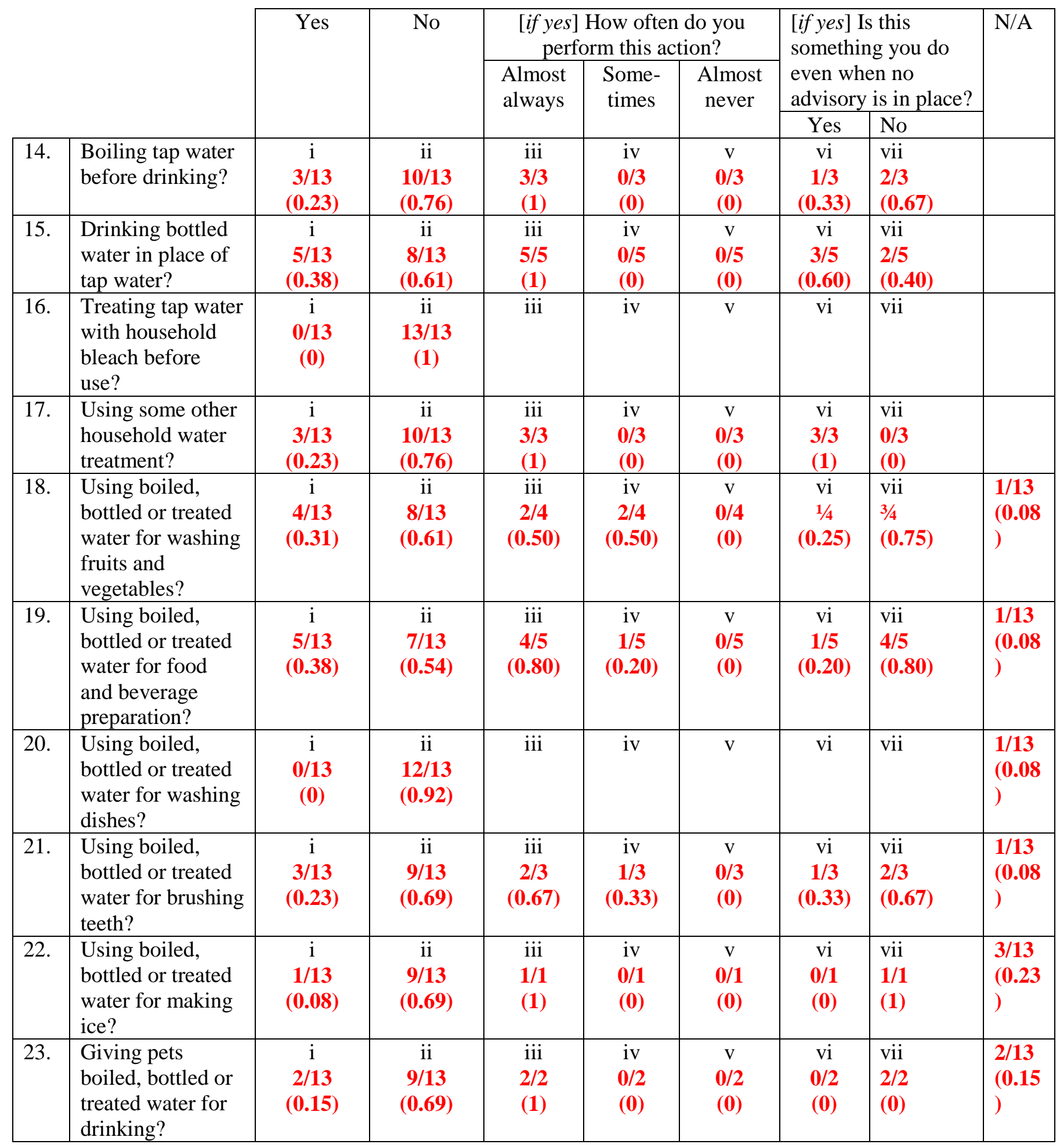


24. Since the boil water advisory was issued, have you been doing anything else differently? i. Yes $1 / 13(0.08)$

ii. No $12 / 13(0.92)$ [if yes] Is there anything you have been doing differently, other than what you have said in the previous questions?

[if no] Why not? 
In the next set of questions, I will read out some statements related to boil water advisories, in general. For each statement, please tell me whether you strongly agree, agree, neither agree nor disagree, whether you disagree or strongly disagree. Percentages provided

\begin{tabular}{|c|c|c|c|c|c|c|c|c|}
\hline & $\begin{array}{l}\mathrm{SA}=\text { strongly agree; } \mathrm{A}=\text { agree; } \mathrm{N}=\text { neutral; } \\
\mathrm{D}=\text { disagree; } \mathrm{SD}=\text { =strongly disagree; } \mathrm{DK}=\text { don't } \\
\text { know; NR= no response/refuse to reply }\end{array}$ & SA & A & $\mathrm{N}$ & D & SD & DK & NR \\
\hline 25. & $\begin{array}{l}\text { My chances of falling sick from not } \\
\text { boiling my tap water is not } \\
\text { significant. }\end{array}$ & $\begin{array}{c}\mathrm{i} \\
23.1\end{array}$ & $\begin{array}{c}\text { ii } \\
30.8\end{array}$ & $\begin{array}{l}\text { iii } \\
7.7\end{array}$ & $\begin{array}{c}\text { iv } \\
23.1\end{array}$ & $\begin{array}{l}\mathrm{V} \\
0\end{array}$ & 0 & 15.4 \\
\hline 26. & $\begin{array}{l}\text { If I don't boil my water before } \\
\text { drinking or use bottled water during } \\
\text { a boil water advisory I will get sick. }\end{array}$ & $\begin{array}{l}\mathrm{i} \\
7.7\end{array}$ & $\begin{array}{c}\text { ii } \\
38.5\end{array}$ & $\begin{array}{l}\text { iii } \\
7.7\end{array}$ & iv & $\begin{array}{c}\mathrm{V} \\
15.4\end{array}$ & 0 & 7.7 \\
\hline 27. & $\begin{array}{l}\text { I take boil water advisories very } \\
\text { seriously }\end{array}$ & $\begin{array}{c}\mathrm{i} \\
15.4\end{array}$ & $\begin{array}{c}\text { ii } \\
38.5\end{array}$ & $\begin{array}{c}\text { iii } \\
23.1\end{array}$ & iv & $\begin{array}{c}\mathrm{V} \\
7.7\end{array}$ & 0 & 0 \\
\hline 28. & $\begin{array}{l}\text { Boil water advisories are issued so } \\
\text { often now-a-days that they have } \\
\text { lost all meaning. }\end{array}$ & $\begin{array}{l}\mathrm{i} \\
0\end{array}$ & $\begin{array}{c}\text { ii } \\
23.1\end{array}$ & $\begin{array}{c}\mathrm{iii} \\
23.1\end{array}$ & iv & $\begin{array}{l}\mathrm{V} \\
0\end{array}$ & 0 & 0 \\
\hline 29. & $\begin{array}{l}\text { If I follow the recommendations } \\
\text { given in boil water advisories, my } \\
\text { drinking water will be safe and I } \\
\text { will not get sick. }\end{array}$ & $\begin{array}{c}\mathrm{i} \\
30.8\end{array}$ & $\begin{array}{c}\mathrm{ii} \\
38.5\end{array}$ & $\begin{array}{c}\text { iii } \\
0\end{array}$ & $\begin{array}{c}\text { iv } \\
15.4\end{array}$ & $\begin{array}{l}\mathrm{V} \\
0\end{array}$ & 7.7 & 7.7 \\
\hline 30. & $\begin{array}{l}\text { Boil water advisories are a good } \\
\text { way of keeping the public safe from } \\
\text { potentially contaminated drinking } \\
\text { water }\end{array}$ & $\begin{array}{c}\mathrm{i} \\
30.8\end{array}$ & $\begin{array}{c}\text { ii } \\
46.2\end{array}$ & $\begin{array}{c}\text { iii } \\
23.1\end{array}$ & iv & $\begin{array}{l}\mathrm{V} \\
0\end{array}$ & 0 & 0 \\
\hline 31. & $\begin{array}{l}\text { The boil water advisory message is } \\
\text { difficult to understand. }\end{array}$ & $\begin{array}{l}\mathrm{i} \\
0\end{array}$ & $\begin{array}{l}\text { ii } \\
7.7\end{array}$ & $\begin{array}{c}\text { iii } \\
0\end{array}$ & iv & $\begin{array}{c}\mathrm{v} \\
30.8\end{array}$ & 0 & 0 \\
\hline 32. & $\begin{array}{l}\text { I can't exactly remember what the } \\
\text { boil water advisory } \\
\text { recommendations are. }\end{array}$ & $\begin{array}{l}\mathrm{i} \\
0\end{array}$ & $\begin{array}{c}\text { ii } \\
53.8\end{array}$ & $\begin{array}{c}\text { iii } \\
0\end{array}$ & $\begin{array}{l}\text { iv } \\
38.5\end{array}$ & $\begin{array}{c}\mathrm{v} \\
7.7\end{array}$ & 0 & 0 \\
\hline 33. & $\begin{array}{l}\text { If I forget to boil my tap water } \\
\text { before drinking, it's no big deal. }\end{array}$ & $\begin{array}{l}\mathrm{i} \\
0\end{array}$ & $\begin{array}{c}\text { ii } \\
23.1\end{array}$ & $\begin{array}{c}\mathrm{iii} \\
30.8\end{array}$ & iv & $\begin{array}{l}\mathrm{V} \\
0\end{array}$ & 0 & 15.4 \\
\hline 34. & $\begin{array}{l}\text { I have no time to boil my tap water } \\
\text { each time before use. }\end{array}$ & $\begin{array}{c}\mathrm{i} \\
15.4\end{array}$ & $\begin{array}{c}\text { ii } \\
15.4\end{array}$ & $\begin{array}{c}\text { iii } \\
15.4\end{array}$ & iv & $\begin{array}{l}\mathrm{V} \\
0\end{array}$ & 0 & 15.4 \\
\hline 35. & $\begin{array}{l}\text { It is a hassle to boil tap water } \\
\text { during boil water advisories, so I } \\
\text { rather not do it. }\end{array}$ & $\begin{array}{c}\mathrm{i} \\
15.4\end{array}$ & $\begin{array}{c}\text { ii } \\
23.1\end{array}$ & $\begin{array}{c}\text { iii } \\
0\end{array}$ & $\begin{array}{c}\text { iv } \\
46.2\end{array}$ & $\begin{array}{l}\mathrm{V} \\
0\end{array}$ & 0 & 15.4 \\
\hline
\end{tabular}




\begin{tabular}{|c|c|c|c|c|c|c|c|c|}
\hline 36. & $\begin{array}{l}\text { I don't understand why boil water } \\
\text { advisories are issued. }\end{array}$ & $\begin{array}{l}\mathrm{i} \\
0\end{array}$ & $\begin{array}{l}\text { ii } \\
0\end{array}$ & $\begin{array}{c}\mathrm{iii} \\
0\end{array}$ & $\begin{array}{l}\text { iv } \\
69.2\end{array}$ & $\begin{array}{c}\mathrm{V} \\
23.1\end{array}$ & 0 & 7.7 \\
\hline 37. & $\begin{array}{l}\text { I will not buy bottled water during } \\
\text { boil water advisories because of the } \\
\text { cost. }\end{array}$ & $\begin{array}{c}\mathrm{i} \\
7.7\end{array}$ & $\begin{array}{c}\text { ii } \\
7.7\end{array}$ & $\begin{array}{l}\mathrm{iii} \\
7.7\end{array}$ & $\begin{array}{c}\text { iv } \\
53.8\end{array}$ & $\begin{array}{l}\mathrm{V} \\
0\end{array}$ & 0 & 23.1 \\
\hline 38. & $\begin{array}{l}\text { I would rather boil water than buy } \\
\text { bottled water }\end{array}$ & $\begin{array}{c}\mathrm{i} \\
15.4\end{array}$ & $\begin{array}{c}\text { ii } \\
30.8\end{array}$ & $\begin{array}{l}\text { iii } \\
7.7\end{array}$ & iv & $\begin{array}{l}\mathrm{v} \\
0\end{array}$ & 0 & 23.1 \\
\hline 39. & $\begin{array}{l}\text { I use bottled water anyway, so boil } \\
\text { water advisories aren't important to } \\
\text { me. }\end{array}$ & $\begin{array}{l}\mathrm{i} \\
0\end{array}$ & $\begin{array}{c}\mathrm{ii} \\
7.7\end{array}$ & $\begin{array}{c}\text { iii } \\
15.4\end{array}$ & $\begin{array}{c}\text { iv } \\
30.8\end{array}$ & $\begin{array}{c}\mathrm{V} \\
23.1\end{array}$ & 0 & 23.1 \\
\hline
\end{tabular}

\begin{tabular}{|c|c|c|c|c|c|c|c|c|}
\hline & & $\mathrm{SA}$ & A & $\mathrm{N}$ & $\mathrm{D}$ & SD & $\mathrm{DK}$ & NR \\
\hline 40. & $\begin{array}{l}\text { The safety of my drinking water is } \\
\text { not a major concern for me. }\end{array}$ & $\begin{array}{c}\mathrm{i} \\
7.7\end{array}$ & $\begin{array}{c}\text { ii } \\
7.7\end{array}$ & $\begin{array}{l}\text { iii } \\
7.7\end{array}$ & $\begin{array}{l}\text { iv } \\
46.2\end{array}$ & $\begin{array}{c}\mathrm{V} \\
30.8\end{array}$ & 0 & 0 \\
\hline 41. & $\begin{array}{l}\text { I have no control over the quality of } \\
\text { my drinking water }\end{array}$ & $\begin{array}{c}\mathrm{i} \\
7.7\end{array}$ & $\begin{array}{c}\text { ii } \\
30.8\end{array}$ & $\begin{array}{l}\text { iii } \\
7.7\end{array}$ & $\begin{array}{c}\text { iv } \\
38.5\end{array}$ & $\begin{array}{c}\mathrm{V} \\
15.4\end{array}$ & 0 & 0 \\
\hline 42. & $\begin{array}{l}\text { I have no control over the safety of } \\
\text { my drinking water }\end{array}$ & $\begin{array}{c}\mathrm{i} \\
7.7\end{array}$ & $\begin{array}{c}\text { ii } \\
23.1\end{array}$ & $\begin{array}{l}\mathrm{iii} \\
7.7\end{array}$ & $\begin{array}{l}\text { iv } \\
30.8\end{array}$ & $\begin{array}{c}\mathrm{v} \\
23.1\end{array}$ & 7.7 & 0 \\
\hline 43. & $\begin{array}{l}\text { I am satisfied with how my } \\
\text { drinking water is managed by those } \\
\text { responsible }\end{array}$ & $\begin{array}{c}\mathrm{i} \\
15.4\end{array}$ & $\begin{array}{c}\text { ii } \\
38.5\end{array}$ & $\begin{array}{c}\text { iii } \\
15.4\end{array}$ & $\begin{array}{c}\text { iv } \\
30.8\end{array}$ & $\begin{array}{l}\mathrm{V} \\
0\end{array}$ & 0 & 0 \\
\hline 44. & $\begin{array}{l}\text { I have a high chance of getting sick } \\
\text { from consuming contaminated } \\
\text { drinking water }\end{array}$ & i & $\begin{array}{c}\text { ii } \\
38.5\end{array}$ & $\begin{array}{l}\text { iii } \\
7.7\end{array}$ & $\begin{array}{c}\text { iv } \\
38.5\end{array}$ & $\begin{array}{c}\mathrm{V} \\
15.4\end{array}$ & 0 & 0 \\
\hline 45. & $\begin{array}{l}\text { The risk of falling ill from drinking } \\
\text { contaminated drinking water is } \\
\text { exaggerated }\end{array}$ & $\begin{array}{c}\mathrm{i} \\
7.7\end{array}$ & $\begin{array}{c}\mathrm{ii} \\
7.7\end{array}$ & $\begin{array}{c}\mathrm{iii} \\
0\end{array}$ & $\begin{array}{l}\text { iv } \\
76.9\end{array}$ & $\begin{array}{l}\mathrm{V} \\
0\end{array}$ & 7.7 & 0 \\
\hline 46. & $\begin{array}{l}\text { Bottled water is safer than boiled } \\
\text { tap water }\end{array}$ & $\begin{array}{l}\mathrm{i} \\
0\end{array}$ & $\begin{array}{l}\text { ii } \\
7.7\end{array}$ & $\begin{array}{c}\text { iii } \\
0\end{array}$ & $\begin{array}{l}\text { iv } \\
76.9\end{array}$ & $\begin{array}{c}\mathrm{v} \\
7.7\end{array}$ & 0 & 7.7 \\
\hline
\end{tabular}


We've reached the final section of the interview. In the last set of questions, I will ask you some general questions about yourself and your household.

47. [Record gender]
i. Male
$6 / 13(0.46)$
ii. Female $7 / 13(0.54)$

48. What is your birth year?

49. What is the total number of number of people who live in your household (including yourself)? 8 households had more than one resident in house [if 2 or more] 49.1. Are there any infants or children aged 5 years or younger in your household?
i. Yes $1 / 8(\mathbf{0}(\mathbf{1 2})$
ii. No $7 / 8(0.88)$

49.2. Are there elderly members aged 65 or over in your household?

i. Yes $2 / 8(0.25)$

ii. No $6 / 8(0.75)$

49.3. Is there anyone in your household with severely weakened immune systems, such as a transplant recipient, an AIDS patient or anyone undergoing cancer chemotherapy?

i. Yes $2 / 8(\mathbf{0 . 2 5})$

ii. No $6 / 8(0.75)$

50. Besides English, what languages are spoken in your household?

51. Do you have any concerns with the safety of your drinking water?

i.Yes $4 / 13(0.31)$

ii. No $9 / 13(0.69)$

[if yes] 51.1. What?

52. Finally, do you have any comments you would like to add, for example, about the interview, this study or about your drinking water?

That's it! Thank you so much for participating in this study.

Would you be interested in seeing the results of this study?

YES | NO

Yes: 11/13, No: 2/13

Thank you again for your time. If you ever have any questions, feel free to call me.

Interview End: am pm 


\section{Appendix D: Photograph from a Water System Visit}

This photograph was taken during an onsite visit to Water Users' Community Water System - 7.

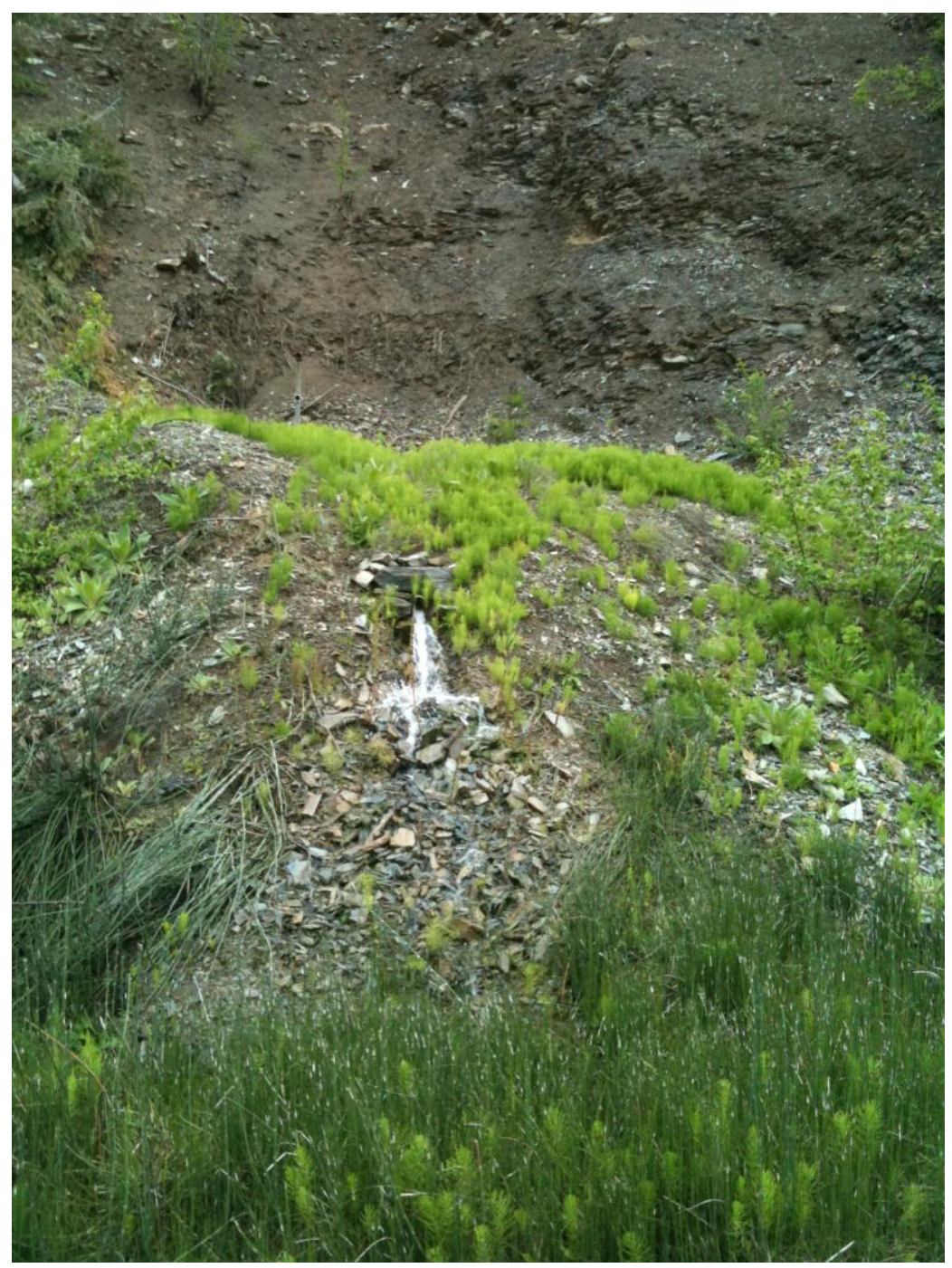

A Description of the source from the EHO:

The source is an underground spring that used to surface out of the hillside. It is not visible above ground now since it was covered over with soil and a pipe was installed that connected the source intake to a buried 500 gallon, concrete reservoir. An underground 3" plastic pipe carries the water from the reservoir under the \#1 Highway to the subdivision on the other side of the highway. The pipe that comes out of the ground close to the highway is an overflow pipe from the reservoir, and this is shown in the photograph above. Since the water flow throughout the system is by gravity, pressure in the distribution system is very low. 